OPEN ACCESS

Edited by:

Yue Liu,

Xiyuan Hospital, China

Reviewed by:

Guanwei Fan,

Tianjin University of Traditional

Chinese Medicine, China

Yanfei Liu,

Beijing University of Chinese Medicine,

China

*Correspondence:

Ali H. Eid

ae81@aub.edu.lb

Gianfranco Pintus

gpintus@sharjah.ac.ae;

gpintus@uniss.it

Gheyath K. Nasrallah

gheyath.nasrallah@qu.edu.qa

Specialty section:

This article was submitted to

Ethnopharmacology,

a section of the journal

Frontiers in Pharmacology

Received: 04 November 2019

Accepted: 19 March 2020

Published: 07 April 2020

Citation:

Shaito A, Thuan DTB, Phu HT, Nguyen THD, Hasan H, Halabi S, Abdelhady S, Nasrallah GK, Eid $A H$ and Pintus G (2020) Herbal Medicine for Cardiovascular Diseases: Efficacy,

Mechanisms, and Safety.

Front. Pharmacol. 11:422.

doi: 10.3389/fphar.2020.00422

\section{Herbal Medicine for Cardiovascular Diseases: Efficacy, Mechanisms, and Safety}

\author{
Abdullah Shaito ${ }^{1}$, Duong Thi Bich Thuan ${ }^{2}$, Hoa Thi Phu ${ }^{2}$, Thi Hieu Dung Nguyen ${ }^{3}$, \\ Hiba Hasan ${ }^{4}$, Sarah Halabi ${ }^{5}$, Samar Abdelhady ${ }^{6}$, Gheyath K. Nasrallah ${ }^{7 *}$, Ali H. Eid ${ }^{7,8^{*}}$ \\ and Gianfranco Pintus ${ }^{9,10 *}$ \\ ${ }^{1}$ Department of Biological and Chemical Sciences, Lebanese International University, Beirut, Lebanon, ${ }^{2}$ Department of \\ Biochemistry, University of Medicine and Pharmacy, Hue University, Hue City, Vietnam, ${ }^{3}$ Department of Physiology, \\ University of Medicine and Pharmacy, Hue University, Hue City, Vietnam, 4 Institute of Anatomy and Cell Biology, Justus \\ Liebig University Giessen, Giessen, Germany, ${ }^{5}$ Biology Department, Faculty of Arts and Sciences, American University of \\ Beirut, Beirut, Lebanon, ${ }^{6}$ Faculty of Medicine, Alexandria University, Alexandria, Egypt, 7 Department of Biomedical Sciences, \\ College of Health Sciences, Qatar University, Doha, Qatar, ${ }^{8}$ Department of Pharmacology and Toxicology, American \\ University of Beirut, Beirut, Lebanon, ${ }^{9}$ Department of Medical Laboratory Sciences, University of Sharjah, Sharjah, United \\ Arab Emirates, ${ }^{10}$ Department of Biomedical Sciences, Faculty of Medicine, University of Sassari, Sassari, Italy
}

Cardiovascular diseases (CVDs) are a significant health burden with an ever-increasing prevalence. They remain the leading causes of morbidity and mortality worldwide. The use of medicinal herbs continues to be an alternative treatment approach for several diseases including CVDs. Currently, there is an unprecedented drive for the use of herbal preparations in modern medicinal systems. This drive is powered by several aspects, prime among which are their cost-effective therapeutic promise compared to standard modern therapies and the general belief that they are safe. Nonetheless, the claimed safety of herbal preparations yet remains to be properly tested. Consequently, public awareness should be raised regarding medicinal herbs safety, toxicity, potentially lifethreatening adverse effects, and possible herb-drug interactions. Over the years, laboratory data have shown that medicinal herbs may have therapeutic value in CVDs as they can interfere with several CVD risk factors. Accordingly, there have been many attempts to move studies on medicinal herbs from the bench to the bedside, in order to effectively employ herbs in CVD treatments. In this review, we introduce CVDs and their risk factors. Then we overview the use of herbs for disease treatment in general and CVDs in particular. Further, data on the ethnopharmacological therapeutic potentials and medicinal properties against CVDs of four widely used plants, namely Ginseng, Ginkgo biloba, Ganoderma lucidum, and Gynostemma pentaphyllum, are gathered and reviewed. In particular, the employment of these four plants in the context of CVDs, such as myocardial infarction, hypertension, peripheral vascular diseases, coronary heart disease, cardiomyopathies, and dyslipidemias has been reviewed, analyzed, and critically discussed. We also endeavor to document the recent studies aimed to dissect the cellular and molecular cardio-protective mechanisms of the four plants, using recently reported in vitro and in vivo studies. Finally, we reviewed and reported the results of the 
recent clinical trials that have been conducted using these four medicinal herbs with special emphasis on their efficacy, safety, and toxicity.

Keywords: herbal medicine, cardiovascular diseases, atherosclerosis, hypertension, medicinal plants, antioxidants, oxidative stress, inflammation

\section{INTRODUCTION}

Cardiovascular diseases (CVDs) are diseases of the heart or blood vessels. CVDs register a global annual toll of more than 17 million deaths. As a result, CVDs remain the world's most common cause of death and are a major economic and health burden, worldwide. The World Health Organization (WHO) reported that CVDs account for $31 \%$ of annual global deaths (World Health Organization, 2017). In Europe, CVDs account for $45 \%$ of all deaths according to the European Cardiovascular Disease Statistics 2017 (Martinet et al., 2019). The American Heart Association's current statistics estimate that around half of the population of the USA has a form of CVD (Benjamin et al., 2019).

CVDs are a variety of diseases including peripheral vascular diseases, coronary heart disease (CHD), heart failure, heart attack (myocardial infarction), stroke, cardiomyopathies, dyslipidemias, and hypertension, among others (Figure 1) (Toth, 2007; Reiner et al., 2019). CVDs majorly originate from a vascular dysfunction, which then leads to organ damage. For example, the heart can suffer a heart attack, or the brain can suffer a stroke due to vascular impairment. Major culprits in vascular impairment include atherosclerosis, thrombosis, and high blood pressure (BP). Common risk factors for CVDs include smoking, unhealthy diet, diabetes mellitus, hyperlipidemia, elevated levels of low-density lipoprotein cholesterol (LDL), suppressed levels of high-density lipoprotein cholesterol (HDL), and hypertension (Figure 1) (World Health Organization, 2017).

CVDs prevention is favored by a healthy vascular endothelium. A healthy endothelium exhibits vasodilatory, anti-atherogenic, and anti-inflammatory properties (Celermajer, 1997). Several risk factors for CVDs lead to endothelial cell (EC) dysfunction, which has been implicated as a key event in the pathogenesis of atherosclerosis, coronary vasoconstriction, and, probably, myocardial ischemia. Interestingly, EC dysfunction is a reversible phenomenon, which opens the door for CVD therapies based on its reversion (Figure 1) (Celermajer, 1997).

Recently, inflammation has been confirmed as a risk factor for CVDs, especially during atherosclerosis and coronary artery disease. High levels of high-sensitivity C-reactive protein (hs-

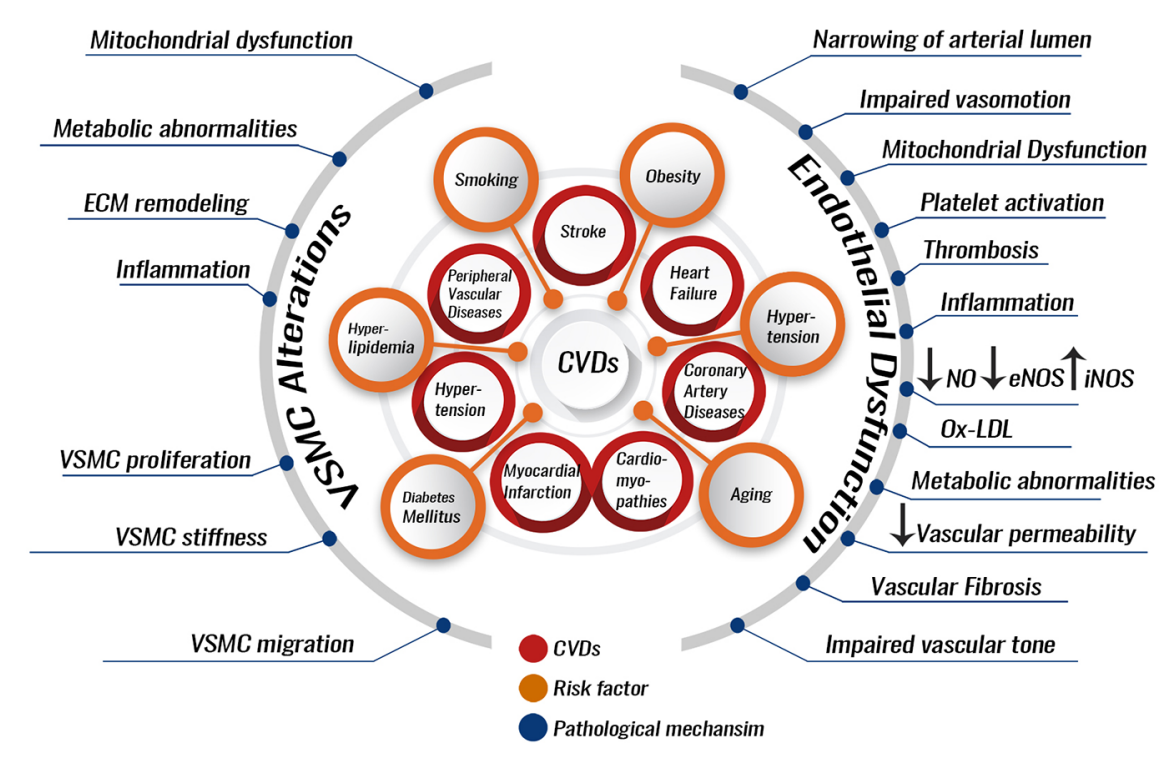

FIGURE 1 | Pathological processes involved in the development and progression of CVDs. Several risk factors can predispose to CVDs. These can include hypertension, smoking, dyslipidemia stemming from an unhealthy diet, or endocrinopathies like diabetes mellitus, hypothyroidism, and aging. The risk factors can lead to pathological alterations most of which can be due to endothelial dysfunction or VSMC alterations. Endothelial dysfunction or VSMC alterations increase the risk of developing atherosclerosis and hypertension. Atherosclerosis and hypertension are themselves CVDs risk factors and enhancers for the development of other CVDs like myocardial infarction, coronary artery diseases, or stroke. VSMC, vascular smooth muscle cell; ECM, extracellular matrix; NO, nitric oxide; eNOS, endothelial nitric oxide synthase; iNOS, inducible nitric oxide synthase; Ox-LDL, oxidized low-density lipoprotein. 
CRP) and/or interleukin-6 (IL-6) are associated with higher absolute cardiovascular risk (Ridker et al., 1997; Ridker et al., 2000), where the CANTOS study, for the first time, established reduced rates of cardiovascular events following an antiinterleukin-1 beta (IL-1 $\beta$ ) based therapy, independent of cholesterol levels (Ridker et al., 2017). Furthermore, common CVDs risk factors, such as diabetes or hypertension, can predispose to CVDs by the mediation of inflammation (Dokken, 2008; Aday and Ridker, 2018).

In the case of atherosclerosis, for example, inflammation can cause EC functional impairment. Dysfunctional ECs allow the accumulation of low-density lipoprotein (LDL) particles in the vessel wall intima where they become modified into oxidized LDL. Oxidized LDL can then activate the dysfunctional ECs to expose cell adhesion molecules (VCAM-1 and ICAM-1) that bind to and recruit inflammatory leukocytes (T-cells and monocytes) into the subendothelial space (Davies et al., 1993; Moore and Tabas, 2011). These inflammatory blood cells secrete interleukins and cytokines, produce reactive oxygen species (ROS) and thus form an inflamed microenvironment within the arterial wall. The inflamed microenvironment promotes vascular smooth muscle cell (VSMC) proliferation, matrix build-up, and lipid deposition, leading to the formation of an atherosclerotic plaque. The monocytes can reach the intima of the vessel, differentiate into macrophages, and uptake oxidized LDL to become foam cells (Tabas et al., 2007; Moore and Tabas, 2011; Douglas and Channon, 2014; Saleh Al-Shehabi et al., 2016; Martinet et al., 2019). Gradual intimal thickening takes place over the years and continues to expand causing decreased or complete occlusion of blood flow to organs, ultimately resulting in CVDs, such as myocardial infarction or stroke (Maguire et al., 2019). In addition, VSMCs proliferation leads to narrowing of the arterial lumen and dysregulation of the vasotone (Douglas and Channon, 2014). Usually several atherosclerotic plaques form in the intima and one of these may end up undergoing a necrotic breakdown, leading to acute luminal thrombosis, blood vessel occlusion, and cardiovascular complications, including myocardial infarction, unstable angina (chest pain caused by heart muscle ischemia), sudden cardiac death, or a stroke (Virmani et al., 2002). As a result, atherosclerosis is not only a risk factor but also a major contributor to CVD incidence. Around $50 \%$ of all deaths in developed countries are due to atherosclerosis (Tedgui and Mallat, 2006).

Hypertension also referred to as high BP, is a CVD and a major risk factor and contributor to other CVDs and other diseases (2017). Hypertension is an independent predisposing factor for heart failure, coronary artery disease, stroke, retinopathy, nephropathy, and peripheral arterial diseases (Sawicka et al., 2011; NCD Risk Factor Collaboration, 2017). Most of these diseases are associated with high mortality and morbidity (Abegaz et al., 2017). Additionally, hypertension is the single most significant risk factor for atherosclerosis, and any clinical outcome of atherosclerosis thereof (Sawicka et al., 2011). Hypertension is a "silent killer" as it does not show symptoms until later stages of the disease (Sawicka et al., 2011). Because of this, it is not surprising that hypertension affects 1.4 billion people and accounts for about 9.4 million deaths per year (Cooper et al., 2017; Egan et al., 2019). Lastly, hypertension prevalence is estimated to have a $30 \%$ worldwide increase by 2025 (Kearney et al., 2005).

The American Heart Association Hypertension Guidelines define hypertension as a persistent elevation of $\mathrm{BP}$ in the arteries [systolic BP (SBP) higher than 130/diastolic BP (DBP) higher than $80 \mathrm{~mm} \mathrm{Hg}$ ] (Muntner et al., 2018). If an elevated BP is left unmanaged, it can induce arterial remodeling; the walls of small vessels thicken, and the vessels lose their elasticity and become narrower. This process is called arteriosclerosis and can lead to "target organ damage" (TOD) (Triantafyllidi et al., 2010; Fan et al., 2017). TOD affects several organs such as the brain, kidney, or retina and may lead to death (Mensah, 2016; Abegaz et al., 2017). Arteriosclerosis can be witnessed in coronary vessels where it may cause a myocardial infarction (Rakugi et al., 1996). In the brain, arteriosclerosis can cause vessel lumen narrowing, vessel wall hardening, and blood clot formation, potentially causing a stroke (Johansson, 1999). Strokes have effects on cognitive and physical behaviors, and may result in dementia, paralysis, or death (Abegaz et al., 2017). Nephrosclerosis of the kidney is also due to arteriosclerosis which stiffens the nephron, ultimately affecting renal filtration and causing electrolyte imbalances (Bidani and Griffin, 2004; Lim et al., 2016). Overall, TOD proceed through hypertension-induced microvascular injuries in the cases of retinopathy and nephropathy and through hypertension-induced macrovascular injuries in the cases of stroke and myocardial infarctions (Nadar, 2011).

$\mathrm{BP}$ regulation, and therefore hypertension, depends on two main factors: cardiac output and systemic vascular resistance. Increased cardiac output or vascular resistance elevate BP. Cardiac output is majorly affected by sodium intake, renal function, and mineralocorticoids. Vascular resistance is affected by the sympathetic nervous system (SNS), rennin-angiotensin system (RAS), humoral factors, and local autoregulation (DiBona, 2013). SNS and RAS exert their effects mainly by eliciting vasoconstriction and inducing sodium retention. Humoral mediators can be vasoconstrictors like endothelin, angiotensin II, catecholamines, or vasodilators, for instance nitric oxide (NO), prostaglandins, and kinins (Oparil et al., 2003). Other factors that can modulate BP include blood flow velocity, blood viscosity, vascular wall stiffness, oxidative stress of VSMCs or ECs, VSMCs proliferation and shape changes, and EC health (Rodrigo et al., 2011).

Despite advances in CVD management and treatment, CVDs still claim more lives than the combination of all cancer forms (Mozaffarian et al., 2015). As a result, in recent years there has been major enforcement on CVD prevention (Reiner et al., 2019). Therefore, new treatment options are urgently warranted for all types of CVDs, considering the continued burden stemming from CVDs is still substantial. 


\section{HERBAL AND PLANT PRODUCTS AS MEDICINAL DRUGS}

Traditional medicine and ethnomedicine, defined as the study of the traditional medicines practiced by various ethnic groups, are as old as human history. Traditional medicine historically relied on natural resources as medications. Historically, herbs, generally defined as any form of plant or plant product (Tachjian et al., 2010), and plant extracts formed the basis of the first drugs used in traditional medicine systems of many cultures and civilizations. Plants and herbs have always been a common source of medications, either in the form of traditional extracts or as pure active compounds (Fabricant and Farnsworth, 2001). Evidently, nature is a very important source for finding new drugs that leads to the treatment of diseases. Famous drugs from herbal and plant sources include aspirin from the Salix alba L. tree, digoxin (cardiac glycoside) from Digitalis purpurea, ephedrine from Ephedra sinica, lovastatin from Monascus purpureus L., taxol from Taxus brevifolia, reserpine from Rauvolfia serpentina, and many others (Harvey, 2000; Frishman et al., 2009; Cragg and Newman, 2013). Interestingly, reserpine is still an effective treatment for hypertension (Weber et al., 2014). Notably, the discovery of antimalarial drugs, quinine from the bark of Cinchona species and artemisinin from Artemisia annua L., represent a typical example of how ethnomedicine can guide drug discovery (Cragg and Newman, 2013).

The earliest records of drugs of natural origin, found in Mesopotamia (from around $2600 \mathrm{BCE}$ ), describe the use of approximately 1000 plant-derived compounds. The best record of using natural extracts in therapy is the Egyptians' Ebers Papyrus (from $1500 \mathrm{BCE}$ ), which documents more than 700 natural drugs, mainly of plant origin. The Chinese Materia Medica record (BCE 1100 ) describes 52 natural medicinal preparations, and the Indian Ayurvedic record (BCE 1000) describes more than 800 natural medicinal extracts (Cragg and Newman, 2013; Otvos et al., 2019). Hippocrates also applied phytotherapy, or healing with herbs, in his treatments (Otvos et al., 2019).

In 1985 WHO estimated that around 65\% of the world population mostly depended on plant-derived traditional medicines (Farnsworth et al., 1985). People in different countries have come to use identical or comparable plants or herbal preparations for the prevention and/or treatment of physical and mental illnesses. Traditional Medicine Centers of the WHO identified 122 compounds to be commonly used in the Center's host countries. Interestingly, the 122 compounds have been reported to derive from only 94 plant species and are used for similar ethnomedical treatments in the different host countries (Farnsworth et al., 1985). Examples of such compounds include galegine, from Galega officinalis L., the base for the synthesis of metformin and similar bisguanidine-type antidiabetic drugs, and papaverine from Papaver somniferum which is the base for making the antihypertensive drug verapamil (Fabricant and Farnsworth, 2001). Commercially, drug production from natural products such as herbs is a viable commodity, where $39 \%$ of the 520 new drugs approved between 1983 and 1994 were natural compounds or derived from natural compounds and $60-80 \%$ of antibacterial and anticancer drugs were derived from natural products in that same period (Harvey, 2000).

Despite the many successes of using natural products for drug production, advances in combinatorial chemistry (in the late 1980s) shifted the focus of drug discovery efforts from natural products to synthesis at the laboratory bench (Cragg and Newman, 2013). This is mainly because natural product-based drug discovery and development is a complex endeavor demanding costly and highly integrated interdisciplinary approaches (Davison and Brimble, 2019; Otvos et al., 2019). Nonetheless, currently the use of natural products as drugs or as drug discovery platforms is "well and alive" (Newman and Cragg, 2016). In fact, traditional herbal and plant-derived extracts are becoming main stream as advances in scientific research are showing their importance in the prevention and treatment of diseases (Frishman et al., 2009).

Numerous and chemically diverse secondary metabolites have been purified from plant bioactives and have been optimized for exerting a biological effect, nonetheless, they are still away from exhaustive investigation for clinical use. However, recent published scientific evidence, technological advances, and research trends clearly point that naturally-derived compounds will be major sources of new drugs (Davison and Brimble, 2019; Otvos et al., 2019). This has provided a driving cause for the renewed popularity of traditional herbal and plant-derived medications among researchers, despite developments in combinatorial chemical synthesis and the production of modern synthetic drugs (Frishman et al., 2009).

Another reason for the regained interest in medicinal plant products is that, in their attempts to control diseases amid scarce socioeconomic resources, rural communities in developing countries have found resort in traditional herbal and plantderived remedies. This is due to several factors, but in particular to the fact that plant-based medicines are a cheaper alternative with fewer side effects (Frishman et al., 2009; Tabassum and Ahmad, 2011). Herbal and plant remedies are not only economical, but they also contain thousands of bioactive components that have known therapeutic applications (Pan et al., 2013). Additionally, because herbs are viewed as food products, they are not subject to the same surveillance and regulation as conventional drugs. Moreover, herbal remedies are viewed by patients as being natural and therefore safe. However, more research efforts are required to validate the efficacy and the safety profile of such medicaments since many have adverse outcomes that can sometimes have life-threatening effects. It should also be noted that there is concern regarding herb-drug interaction (Tachjian et al., 2010; Anwar et al., 2016; Yuan et al., 2016).

One more reason for the revived interest in natural products is that the biological activity and structural diversity of natural products are unmatched by any available synthetic drug screening library (Davison and Brimble, 2019). Natural products have been selected by nature for bioactivity, over millions of years. As a result, natural product screening libraries need not be superfluously big, as is the case with synthetic drug screening libraries. In addition, natural products need only minor structural changes to optimize their drugability (Harvey, 2000; Gerry and Schreiber, 2018). As such, natural products offer "privileged scaffolds" and serve as 
biologically "pre-validated platforms" for the design of compound candidate drug libraries (Davison and Brimble, 2019). Recent progress has focused on improving the potency, selectivity, and pharmacokinetics of bioactive natural products through structural modifications, which has led to the production of novel drug-like lead compounds. These structural changes are often required, as natural products usually show unfavorable toxicities and pharmacokinetics, limiting their clinical potential (Gerry and Schreiber, 2018; Davison and Brimble, 2019). Overall, natural products have been the single most productive source of drug leads even though little of nature's biodiversity has been tested for biological activity yet (Harvey, 2000).

We therefore address and expose the general rationale for using medicinal herbs in the therapy of diseases in general and CVDs in particular. Then, we move to discuss the medicinal potentials of four traditional herbs (Ginseng, Ginkgo biloba, Ganoderma lucidum, and Gynostemma pentaphyllum) for the treatment of CVDs, which are getting increasing popularity due to their commercial commodity in many markets worldwide and to their proven therapeutic potential in several settings including cardiovascular conditions. We describe and critically discuss their therapeutic benefits in terms of molecular, cellular, and metabolic properties in the context of CVDs. In addition, we highlight the major clinical trials in which these four herbs have been used, with an emphasis on their efficacy and safety.

\section{MODERN MEDICINE MANAGEMENT OF ATHEROSCLEROSIS AND HYPERTENSION}

Current health care guidelines emphasize prevention to minimize the risk of CVDs (Reiner et al., 2019). This is carried out by addressing the major CVD risk factors and trying to minimize their adverse outcomes. In atherosclerosis, most therapeutic approaches aim to control hypertension and hyperlipidemia or modulate hemostasis in order to avoid thrombotic complications (Weber and Noels, 2011). Hypercholesterolemia is a major contributor in atherosclerosis, so current conventional therapeutic approaches rely significantly on lowering LDL levels using statins (Ridker et al., 2017; Aday and Ridker, 2018; Reiner et al., 2019). In cases where statin therapy does not yield a significant reduction in LDL levels, an LDL-absorption inhibitor can be used, alone or in combination with statins depending on patient response. Clinical trials have clearly shown that such therapies are effective in lowering CVD risk (Reiner et al., 2019). Recently, pro-protein convertase subtilisin/kexin type 9 (PCSK9) inhibitors were approved by the regulatory bodies as a drug that can lower LDL level, and are recommended for use in patients with heart problems, where statins were not effective at lowering LDL levels (Ridker et al., 2017; Aday and Ridker, 2018). The CANTOS clinical trial (2017) has provided evidence that in patients with elevated inflammation (hsCRP > $2 \mathrm{mg} / \mathrm{L}$ ), a combination therapy of statins and canakinumab (IL-1 $\beta$ antibody) may be necessary to lower atherosclerosis risk (Ridker et al., 2017; Aday and Ridker,
2018; Reiner et al., 2019). Prior to the recommendations of the CANTOS study, conventional therapy regimens have neglected the role of inflammation in atherosclerosis (Weber and Noels, 2011). It is very important to highlight that complementary and alternative medicine (CAM), including herbal remedies, have already tackled the inflammatory arm of atherosclerosis much earlier than the results of the CANTOS study (Frishman et al., 2009; Orekhov et al., 2013; Al-Shehabi et al., 2016), giving a hint as to why American patients visited CAM providers much more than primary care physicians (Eisenberg et al., 1998; Tachjian et al., 2010). Of relevance to this discussion is that herbal remedies are the most common type of CAM among CVD patients (Yeh et al., 2006; Tachjian et al., 2010).

Modern therapy regimens for hypertension involve controlling BP elevations in hypertensive patients. This usually requires the use of multiple antihypertensive drug agents in the majority of these patients (Guerrero-Garcia and Rubio-Guerra, 2018). Multiple classes of antihypertensive agents are available thus offering a practitioner the ability to prescribe highly effective drug combinations in order to reduce BP and protect target organs (Stewart et al., 2019). This combination therapy is of distinctive importance in resistant hypertension, which is highly prevalent worldwide (Noubiap et al., 2019; Samaha et al., 2019).

The major drug classes available for the management of hypertension are thiazide diuretics, angiotensin-converting enzyme (ACE) inhibitors, angiotensin receptor II blockers, and calcium channel blockers (Susalit et al., 2011; Munoz-Durango et al., 2016). Vasodilators, aldosterone antagonists, $\beta$-blockers, $\alpha$-blockers, renin inhibitors, and central-acting agents are other agents that are occasionally used (Omboni and Volpe, 2018). These agents lower BP in patients and reduce their risk of hypertension-related CVD events, but do not prevent them thereby justifying the use of hypertension combination therapies (Rizvi, 2017).

Despite the availability of the aforementioned medications in modern-day health care systems, high BP is managed in only $34 \%$ of the patients (August, 2004; Wang and Xiong, 2012). Such an aspect appears to be mainly related not only to the cost of antihypertensive agents (Susalit et al., 2011), but also to their availability and accessibility (Wang and Xiong, 2012), their unwanted side effects (Susalit et al., 2011; Wang and Xiong, 2012), and their low patient compliance with the required dose (August, 2004). For these factors hypertension patients seek CAM medications, especially herbal-based medicaments to treat their CVDs in general and hypertension in particular (Yeh et al., 2006; Tachjian et al., 2010; Al Disi et al., 2016).

\section{HERBAL MEDICINE MANAGEMENT OF ATHEROSCLEROSIS AND HYPERTENSION}

Herbal extracts and their derivatives can favorably modulate and ultimately ameliorate the molecular events that contribute to hypertension or atherosclerosis, the two major contributors to CVDs incidence. Herbal remedies contain numerous bioactives 
and, thus, have multi-modal cellular mechanisms of action. In fact, herbal remedies can have antioxidant, vasorelaxant, antiinflammatory, anti-proliferative, or diuretic effects. Herbal remedies can also prevent VSMC phenotypic switching, inhibit endothelial dysfunction, platelet activation, lipid peroxidation, ROS production, and macrophage atherogenicity. Because of such a wide range of molecular and cellular targets, herbal preparations can be used to treat and manage a range of CVDs. For example, Salvia miltiorrhiza (Red sage), an annual sage traditionally used in Chinese medicine, has been used to treat a plethora of CVDs including CHD, myocardial infarction, atherosclerosis, and angina pectoris. The active compounds are mainly utilized as the dried root of the plant rhizome named Danshen (Gao et al., 2012). The plant bioactive compounds are the lipo-soluble Tanshinones and the water-soluble Phenolics (Ren et al., 2019). S. miltiorrhiza extracts have shown strong antioxidant capabilities with a high ability to scavenge free radicals, which seems the base of its strong cardio- and vascular-protective potential (Zhao et al., 2006).

Salvianolic acid B, one of the pure compounds that can be extracted from S. miltiorrhiza, is effective against fibrosis and ischemia-reperfusion injury (Lay et al., 2003). Danshen has a protective effect against homocysteine-induced adverse effects, where homocysteine imbalance is a high-risk factor for vascular diseases (Chan et al., 2004). In combination with Pueraria montana var. lobata. (Kudzu), Danshen has showed potent anti-hypertensive effects ( $\mathrm{Ng}$ et al., 2011). In one clinical trial, Danshen capsules (1000 mg twice daily for 12 weeks) were able to significantly reduce SBP and pulse rate in patients with uncontrolled mild to moderate hypertension and under conventional antihypertensive treatment. It has also been found to be well-tolerated and considered to be safe in patients with hypertension (Yang et al., 2012).

Astragalus membranaceus (Synonym Astragalus propinquus Schischkin. in the Missouri Botanical Garden plant list), another Chinese herb, contains Astragaloside IV, which is the plant major bioactive compound widely used as an antioxidant and for protection against ischemic-associated CVDs (Zhang et al., 2006). A. membranaceus extract has been found to maintain cardiac functions by improving energy metabolism and inhibiting the production of free radicals in a myocardial ischemia reperfusion rat model (Zhou et al., 2000). By decreasing the levels of the oxidative stress marker malondialdehyde (MDA), maintaining superoxide dismutase (SOD) activity, and reducing free radicals-induced myocardial cell injury, $A$. membranaceus can also improve cardiac function and provide cardioprotection in a myocardial ischemic rat model (Ma et al., 2013). A. membranaceus extract also has angiogenic effects in the ischemic injury rat model (Zhang et al., 2011). Astragaloside IV has been found to provide a positive inotropic effect improving left ventricular ejection in patients with congestive heart failure (CHF) (Luo et al., 1995). The polysaccharide of $A$. membranaceus has also been shown to reduce insulin resistance and to possess anti-obesity and hypolipidemic effects (Mao et al., 2009).

Allium sativum (Garlic) is a classic example of herbs used in CVDs management and is quite known for its multifaceted properties against CVD-associated conditions such as hypertension, oxidative stress, inflammation, and hyperlipidemia (Ashraf et al., 2013; Jeong et al., 2016; Thomson et al., 2016). Indeed, by reducing total cholesterol and LDL levels, decreasing the content of lipid in arterial cells and inhibiting VSMCs proliferation, garlic can be used to manage atherosclerosis and hyperlipidemia (Sun Y. et al., 2018). Owing to its endothelial NO synthase (eNOS)modulated vasorelaxation ability, Crataegus oxyacantha (Synonym Crataegus rhipidophylla Gand. Common name Hawthorn) is another example of herbs commonly used to manage hypertension (Brixius et al., 2006). Another herb, Crocus sativus (Saffron), can block $\mathrm{Ca}^{2+}$ channels via endothelium-independent mechanisms providing another vasodilator mechanism, in addition to its eNOS activating ability (Razavi et al., 2016). Among other medicinal plants Hibiscus sabdariffa (roselle), is known to reduce BP using its ability to inhibit ACE (Ojeda et al., 2010), while Camellia sinensis (Tea) extracts can reduce hypertension by significantly increasing brachial artery flow-mediated dilation (FMD) (Ras et al., 2011). Rosemary (Rosmarinus officinalis) exhibits neuroprotection by acting against ischemic stroke-associated cerebral insufficiency, which is characterized by a reduction of localized blood flow in the brain. Through its anti-inflammatory properties, rosemary can decrease the expression of inducible NO synthase (iNOS) and cyclooxygenase-2 (COX-2) as well as that other pro-inflammatory enzymes and mediators (Seyedemadi et al., 2016). The use of herbal plants extends to include $\mathrm{CHF}$ and atrial arrhythmias. Digitalis, extracted from the dried leaves of the common foxglove, is a potent inhibitor of $\mathrm{Na}^{+} / \mathrm{K}^{+}$-ATPase and can cause depolarization leading to smooth muscle contraction and vasoconstriction and hence can strengthen muscle heart contractions (Liu et al., 2016).

Given all these restorative abilities, it is not surprising that herbal remedies are being absorbed into evidence-based medicine for the prevention and/or treatment of CVDs. Table 1 lists common herbal remedies and the form of CVDs that they can help alleviate.

Although herbs have been widely used in both traditional and modern medicine, a limited number of reviews that gather them and comprehensively focus on their mechanisms of action and safety in the context of CVDs are present. Many plant-based compounds appear to have cardiovascular protective effects, nevertheless, among the most effective compounds are flavonoids, terpenoids, saponins, and polysaccharides. These highly effective compounds are major components of four of the most recognized herbal preparations namely: Ginseng, Ginkgo biloba, Ganoderma lucidum, and Gynostemma pentaphyllum, which we decide to cover in this review. 
TABLE 1 | Some herbal remedies traditionally used for the treatment of different forms of CVDs.

\begin{tabular}{|c|c|}
\hline CVD form & Examples of herbal remedies used \\
\hline \multirow{4}{*}{$\begin{array}{l}\text { Atherosclerosis and } \\
\text { hyperlipidemia }\end{array}$} & Garlic (Allium sativum) \\
\hline & Commiphora mukul \\
\hline & Monascus purpureus \\
\hline & Berberine (active compound of Coptis chinensis) \\
\hline \multirow[t]{20}{*}{ Systolic hypertension } & Garlic (Allium sativum) \\
\hline & Rauvolfia serpentina \\
\hline & Panax species (Ginseng) \\
\hline & Stephania tetrandra \\
\hline & Veratrum species alkaloids \\
\hline & $\begin{array}{l}\text { Ligusticum wallichii (Synonym of Ligusticum } \\
\text { striatum DC) }\end{array}$ \\
\hline & Hawthorn from Crataegus oxyacantha \\
\hline & Camelia sinensis \\
\hline & Andrographis paniculata \\
\hline & Apium graveolens \\
\hline & Bidens pilosa L. \\
\hline & Crocus sativus \\
\hline & Cymbopogon citratus \\
\hline & Hibiscus sabdariffa \\
\hline & Nigella sativa \\
\hline & Urtica dioica \\
\hline & Viola odorata \\
\hline & Mentha Iongifolia \\
\hline & Salvia miltiorrhiza \\
\hline & Uncaria Rhynchophylla \\
\hline \multirow[t]{2}{*}{ Venous insufficiency } & Aesculus hippocastanum \\
\hline & Ruscus aculeatus \\
\hline \multirow[t]{2}{*}{ Cerebral insufficiency } & Ginkgo biloba \\
\hline & Rosmarinus officinalis \\
\hline \multirow[t]{3}{*}{ Angina pectoris } & Crataegus species \\
\hline & Panax notoginseng \\
\hline & Salvia miltiorrhiza \\
\hline \multirow[t]{6}{*}{ Congestive heart failure } & Digitalis purpurea \\
\hline & Digitalis lanata \\
\hline & Crataegus species \\
\hline & Adonis microcarpa and Adonis vernalis \\
\hline & Berberine (active compound of Coptis chinensis) \\
\hline & Salvia miltiorrhiza \\
\hline
\end{tabular}

The herbal remedies refer to the plant extract, unless otherwise indicated where the remedy may be a purified or partially purified active ingredient of the plant extract. Data were obtained from published data in Nalli and Giardina, 2002; Frishman et al., 2009; Al Disi et al., 2016; Al-Shehabi et al., 2016; Samaha et al., 2019).

\section{GINSENG}

Ginseng is an anciently cultivated plant (2000 years ago) partly due to its ritual use (Figure 2). Ginseng use in traditional medicine goes back to 20 centuries ago (Kim, 2012), but its use in Western medicine dates back to the early $20^{\text {th }}$ century by two British physicians F. Porter Smith and G.A. Stuart who were exploring Chinese herbal remedies at the time (Shih-Chen et al., 1973). Ginseng habitats include Asian countries such as Korea, China, Japan, and Vietnam, and North American countries, mainly Canada and the United States. Korean red ginseng (KRG; Panax ginseng C.A. Mey.), Chinese ginseng (Panax notoginseng Burkill; F.H.Chen.), American ginseng (Panax quinquefolium L.), and Japanese ginseng (Panax japonicas C.A. Mey.) represent the most commonly used ginsengs.
Usually the roots of 5 to 7-year-old plants are either air-dried under the sun yielding "white ginseng" or steam-treated at 98 $100^{\circ} \mathrm{C}$ for $2-3 \mathrm{~h}$ and then sun-dried to produce the "red ginseng" (Kim et al., 2000). During steaming, ginseng chemical constituents undergo changes that make red ginseng more pharmacologically effective than white ginseng (Kim et al., 2000). Currently, ginseng is prepared and used either in a liquid form: oil extracts or tea; or in a solid form: tablets, capsules, or dried roots (Valli and Giardina, 2002). However, extracts of ginseng root, berry, and leaf have been repeatedly demonstrated to have anti-obesity, anti-hyperglycemic, antihypertensive, insulin sensitization, and anti-hyperlipidemic effects (Kim, 2012).

More than 300 bioactives have been isolated from Ginseng. Ginsenosides, which are triterpene saponins, are the most bioactive constituents isolated from Ginseng extracts (Mahady et al., 2000). Of the 40 ginsenosides isolated so far, Rb1, Rg1, Rg3, Re, and Rd are the most frequently studied. Rg3, Rg5, and RK1 are unique to the red Korean Ginseng (Figure 2) (Lee and Kim, 2014). Research into Ginseng and its constituents has flourished so that currently there is a journal dedicated to Ginseng research: Journal of Ginseng Research (https://www.journals.elsevier.com/journal-of-ginsengresearch). The study of the purified individual ginsenosides rather than the whole Ginseng root extract has gained recent interest (Kim, 2012). Ginseng and its ginsenoside constituents have vasorelaxation, anti-oxidation, anti-inflammation, and anticancer activities (Kim, 2012; Choi J. et al., 2013).

\section{Ginseng at the Bench: Mechanism of Action in CVDs}

In the context of CVDs, Ginseng has been used to manage hypertension. Ginseng has hypotensive effects due to its effect in the improvement of arterial functions. Interestingly, ginsenosides facilitate vasorelaxation of different vessels: rat aortas (Kim et al., 1999a), murine coronary arteries (Pan et al., 2013), and monkey cerebral arteries (Toda et al., 2001). Ginseng can increase eNOS expression and NO production while ginsenoside Rg3 activates eNOS (Valli and Giardina, 2002; Jang et al., 2011; Hong et al., 2012; Pan et al., 2013; Lee K. et al., 2016). KRG induces NO-dependent vasorelaxation improving vascular tone. These effects are mediated by the inhibition of arginase activity, the increase of NO generation, and the enhancement of eNOS dimer formation (Shin W. et al., 2013). The Panax ginseng G115 extract has also been shown to inhibit ACE activity in human umbilical vein endothelial cells (HUVECs) and angiotensin I-induced contractions of bovine mesenteric arteries (Persson et al., 2006). Other Ginseng CVDs management properties are its anti-oxidant (Lee et al., 2019a), anti-inflammatory (Keum et al., 2003; Shin Y. et al., 2013), and anti-hyperlipidemic (Park et al., 2005) effects, along with its ability to regulate $\mathrm{Ca}^{2+}$ channels (Lee and Kim, 2014).

The ginsenoside $\mathrm{Rg} 3$ can increase $\mathrm{NO}$ and cGMP levels, activate $\mathrm{Ca}^{2+}$-gated potassium channels, inhibit ACE activity, and block $\mathrm{Ca}^{2+}$-gated channels (Kim et al., 1999b; Persson et al., 2006; Park J. et al., 2014). Ginseng has also demonstrated an antiinflammatory role by inhibiting the activation of activator 


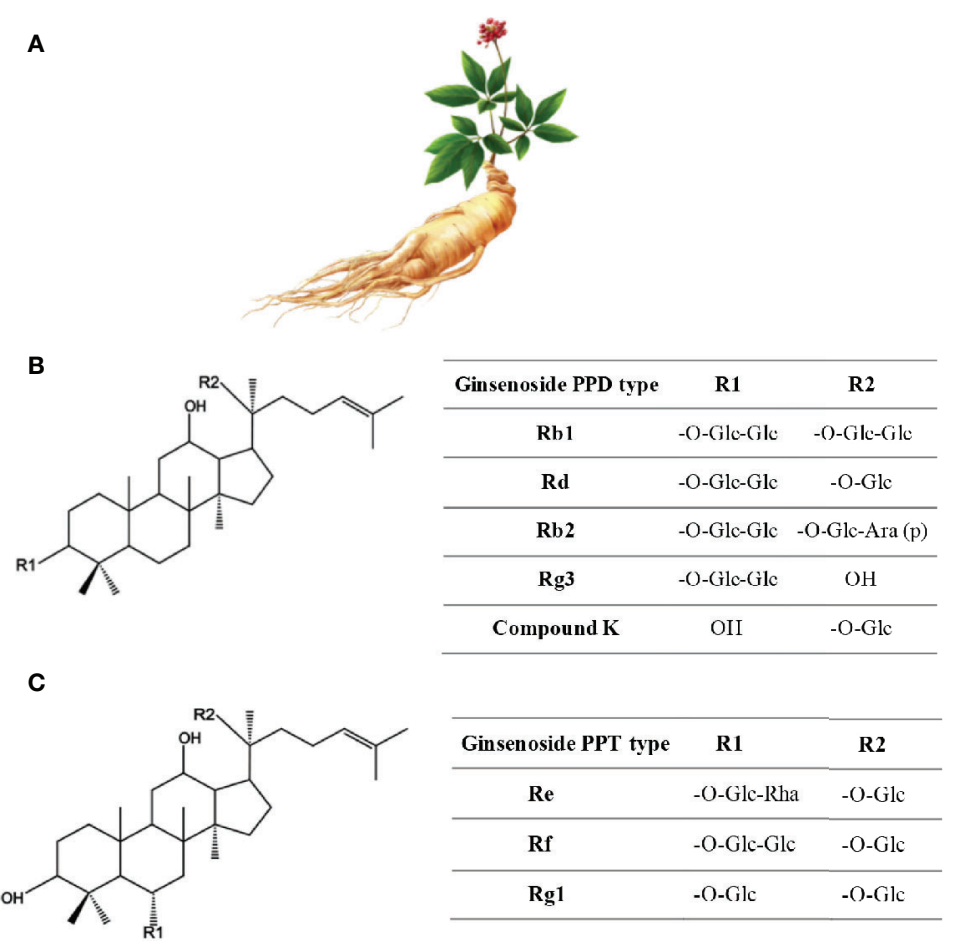

FIGURE 2 | Ginseng. (A) Roots have the healing properties (from https://pngtree.com/freepng). (B) Chemical structure of Ginsenosides. (C) Ginsenosides protopanaxadiol (PPD) and Ginsenoside protopanaxatriol (PPT). R1 and R2 are side chains in different ginsenosides. Glc, glucose; Ara, arabinose; Rha, rhamnose.

protein (AP-1) and nuclear factor-kappa B (NF- $\mathrm{KB})$, ultimately reducing the expression of COX-2, IL-6, IL-1 $\beta$, and tumor necrosis factor- $\alpha$ (TNF- $\alpha$ ) (Keum et al., 2003; Shin Y. et al., 2013). In macrophages, Baek et al. demonstrated that each fraction of the KRG exerts anti-inflammatory actions through a different mechanism. For instance, the saponin fraction significantly suppressed NO production and reduced the expression of inflammatory genes such as iNOS, COX-2, TNF$\alpha$, and interferon- $\beta$. In contrast, all extracts, including water extracts, saponin, and non-saponin fractions, inhibit the activity of the kinase TBK1 and suppress both nuclear translocation and transcriptional activity of its downstream effector interferon regulatory factor 3 (IRF3) (Baek et al., 2016).

By inhibiting diacylglycerol liberation, dietary supplementation of KRG lowers blood cholesterol levels and reduces the formation of atherosclerotic lesions induced by a high cholesterol diet in rabbit (Hwang et al., 2008). Again, by up-regulating the adenosine triphosphate-binding cassette transporter Al, the saponin fraction of $P$. notoginseng can attenuate cholesterol esters in foam cells (Jia et al., 2010). In addition, Ginseng has shown a potent in vivo antithrombotic effect, which may be due to an antiplatelet activity rather than an anticoagulation activity, indicating that Ginseng intake may be beneficial for individuals with high risks of thrombosis and CVDs (Lee and Kim, 2014). In this context, the dihydro-ginsenoside Rg3 has been reported to potently inhibit platelet aggregation through the modulation of downstream intracellular signals such as cAMP and extracellular signal-regulated kinase 2 (Lee et al., 2008).

\section{In Vivo Preclinical Evaluation of Ginseng in Animal Models of CVDs}

Ginseng's hypotensive effects have been extensively demonstrated (Valli and Giardina, 2002; Lee et al., 2012; Mucalo et al., 2013; Al Disi et al., 2016). For example, Ginseng can reduce adrenal catecholamines secretion in hypertensive rats, thus contributing to vasorelaxation (Jang et al., 2011). However, there are reports of Ginseng being hypertensive (Jang et al., 2011; Kim, 2012). In fact, Ginseng may have biphasic concentrationdependent effects. Low doses of ginseng raise BP, while higher concentrations repress BP (Jang et al., 2011), a phenomenon that could be due to the varied action of different Ginseng extract constituents (Valli and Giardina, 2002).

Through their antioxidant properties, ginsenosides also mediate anti-hypertensive and anti-atherosclerotic effects. Ginsenosides exhibit free radical scavenging and metal ion chelating abilities. For instance, the lipid-soluble and water-soluble extracts from the North American Ginseng exhibit strong antioxidant activity (Kitts et al., 2000). Lu et al. showed that Rb1 could significantly and specifically alleviate hydroxyl radical and hypochlorous acid radical damaging effects (Lu et al., 2012). Aged rats, supplemented with the North American Ginseng for four months, had decreased production of both ROS and age-related oxidative damage in proteins of the heart and muscle fibers, a phenomenon mediated by SOD and glutathione peroxidase (GSH-Px) activation (Fu and Ji, 2003). The ginsenoside Rg3 can ameliorate mitochondrial dysfunction and promote enhanced expression of antioxidant 
proteins, such as the nuclear factor erythroid 2-related factor-2 (Nrf2) and the heme oxygenase-1 (HO-1) (Lee et al., 2019a). By reducing hypertension- and atherosclerosis-associated inflammatory states, Ginseng can reduce CVD risk. To address the anti-inflammatory effects of Ginseng, Mohammadi et al. carried out a meta-analysis of data from randomized controlled trials. They report that Ginseng supplementation significantly lowered the levels of two key pro-inflammatory mediators IL- 6 and TNF- $\alpha$ (Mohammadi et al., 2019). By modulating angiogenesis (decreased VEGF-A and FGF-2 expression), inflammatory (decreased CD68, TNF $\alpha$, and MCP-1 expression) and matrix metalloproteinase (MMP) activity, Ginseng can inhibit ovariectomy-induced obesity, adiposity, and adipocyte hypertrophy (Lee H. et al., 2016).

Ginseng can lower the risk of atherosclerosis by inducing a better lipid profile. Ginseng's beneficial effects on lipid metabolism have been described more than three decades ago (Qureshi et al., 1983; Yamamoto et al., 1983). In humans and rats, red Ginseng supplementation improves lipid profiles by diminishing the total plasma levels of cholesterol, triglycerides, LDL-C, free fatty acids (FFA), and platelet adhesiveness and increasing HDL-C levels in total plasma (Deng et al., 2017; Singh et al., 2017). In rats, black Ginseng can ameliorate hypercholesterolemia by interfering with the expression of cholesterol metabolism genes (Saba et al., 2016). In addition, lipid profiles tend to improve in diabetic rats receiving Ginseng, suggesting that Panax ginseng can ameliorate diabetes mellitus-initiated dyslipidemias (Deng et al., 2017; Abdelazim et al., 2018). By modulating the secretion of lipoproteins, Ginseng can reduce the microsomal triglyceride transfer protein (MTTP) (Oh et al., 2012), which plays an essential role in lipid metabolism and transport (Deng et al., 2017).

KRG works as an agonist of peroxisome proliferator-activated receptor (PPAR), which is known to improve atherogenic dyslipidemia by augmenting liver PPAR- $\alpha$ mRNA and raising lipoprotein lipase mRNA levels (Park et al., 2005). Consistent with this study, Shin et al. demonstrated that Ginseng could prevent obesity and dyslipidemia in high-fat diet (HFD)-fed castrated mice. These processes were mediated through the inhibition of adipogenesis-related genes expression (SREBP-1C, PPAR- $\gamma$, FAS, SCD1, and ACC1) in visceral adipose tissues (Shin and Yoon, 2018). Ginseng extracts or ginsenosides can act synergistically with testosterone to further inhibit dyslipidemia (Shin and Yoon, 2018). Mollah et al. also showed that Ginseng can improve lipid profiles via PPAR pathway activation (Mollah et al., 2009; Yang and Kim, 2015). Further, ginsenoside Rg1 can activate the promoter of PPAR- $\alpha$ leading to the expression of its target genes carnitine palmitoyltransferase-1 (CPT-1) and acylCoA oxidase (ACO), which are involved in fatty acid oxidation. These findings indicate that Rg1-induced improvement of lipid profiles may be associated with increased fatty acid oxidation through PPAR- $\alpha$ activation (Park et al., 2011).

\section{Ginseng to the Clinic}

Numerous clinical trials have been conducted to assess the cardio-protective and beneficial effects of Ginseng and its constituents in CVD treatment. Checking the clinical trials on
Ginseng, its extracts or ginsenosides on the U.S. National Library of Medicine www.clinicaltrials.gov reveals that there are 162 Ginseng clinical trials. Of the 162 trials, 47 were Phase 3 or 4 trials, 97 have been completed and the rest are ongoing. Importantly, a significant number of these trials addresses CVDs. For example, eight trials addressed hypertension, five addressed arterial occlusive diseases, and another five addressed strokes. One such clinical trial examined the vasorelaxation effects of Asian Ginseng (AG) and its ability to modulate vascular function. Trial participants were randomized to either the selected AG extract or placebo groups and received a daily dose of $3 \mathrm{~g}$ of AG for 12 weeks in combination with their usual antihypertensive and anti-diabetic therapy. Combining AG extract with conventional therapy in diabetics patients with concomitant hypertension decreased arterial stiffness and attenuated SBP (Mucalo et al., 2013). Another clinical trial found that Rg3 from KRG lowers central and peripheral arterial pressures in healthy adults (Jovanovski et al., 2014). In an acute, randomized, placebo-controlled, double-blind, crossover trial on participants with type 2 diabetes mellitus (T2DM), Shishtar et al. showed that acute administration of Korean white Ginseng appears to be safe and exhibits beneficial effects on the augmentations index, a cumulative indicator of arterial health (Shishtar et al., 2014a).

A 12-week intervention with KRG was conducted in patients with impaired fasting glucose, impaired glucose tolerance, or newly diagnosed with T2DM. Subjects were randomized in a double-blind, placebo-controlled trial. The trial results showed that 12 weeks of intervention with KRG supplementation ( $5 \mathrm{~g} /$ day) led to normalization of whole blood and serum glucose levels as well as serum insulin and CRP concentrations (Bang et al., 2014). Administration of Panax Ginseng extract (PGE) for 8 weeks (6 g/day) decreased serum triglycerides and total cholesterol and LDL levels, while increasing HDL levels. These results were attributed to PGE potent antioxidant effects (Kim and Park, 2003). In accordance, the effects of a low-dose ( $3 \mathrm{~g} /$ day) and a high-dose (6g/day) of KRG supplementation for 8 weeks on antioxidant enzymes and oxidative stress markers in humans were assessed in a randomized, double-blind, placebocontrolled trial. Increased GSH-Px, SOD, and CAT activities were found in the high-dose group as compared to the placebo group. Plasma oxidized-LDL levels and DNA tail length and tail moment were significantly decreased in both high and low dose groups but increased in the placebo group. This led to the conclusion that supplementation with KRG upregulates antioxidant enzymes activities and consequently attenuates lymphocyte DNA damage (Lee et al., 2012).

The efficacy of Ginseng against T2DM is well documented. A meta-analysis of eight clinical trials showed that administration of Ginseng, in comparison to the placebo, improves fasting glucose levels, postprandial insulin levels, and insulin resistance. In these patients, ginseng was able to improve blood lipid profile lowering triglycerides, total cholesterol, and LDL levels. This meta-analysis concluded that Ginseng supplementation can improve the control of glucose levels and insulin sensitivity in patients with T2DM (Gui et al., 2016). Another meta-analysis of 16 randomized clinical trials 
was conducted to assess the efficacy of Ginseng in controlling glycemic index by reporting the ability of Ginseng to reduce fasting blood glucose in both patients with and without diabetes (Shishtar et al., 2014a; Shishtar et al., 2014b). Interestingly, when Ginseng is combined with conventional drugs, its efficacy in the management of hypertension is more pronounced. Indeed, combining AG extract with conventional therapy in diabetic patients with concomitant hypertension decreased arterial stiffness and attenuated SBP (Mucalo et al., 2013).

Despite the numerous clinical trials showing the efficacy of Ginseng in CVDs management, this aspect yet remains controversial. In fact, some studies could not demonstrate Ginseng's beneficial effects against CVD. For example, a metaanalysis of 17 randomized clinical trials (1381 participants) found no significant effect of AG on arterial BP and hence no effect on CVDs risk (Komishon et al., 2016). Another clinical trial concluded that KRG intake (3 g/day) for 3 weeks had no beneficial effects on arterial stiffness in subjects with hypertension (Rhee et al., 2011). Yet, as mentioned above, when KRG was combined with conventional therapy it was able to control hypertension (Rhee et al., 2011).

Thus, Ginseng appears to be efficient in regulating several lipid profile parameters, and has shown positive effects in patients with T2D. Also, the efficacy of Ginseng in the management of hypertension is well documented when combined with conventional hypotensive medications.

\section{Safety, Toxicity, and Side Effects of Ginseng}

As mentioned earlier, the claimed safety of medicinal herbs has to be handled cautiously, and on a case-by-case basis for each herbal preparation. The safety of Ginseng has been experimentally approached using animal models and human clinical studies (Mahady et al., 2000). An abundant number of in vitro and in vivo studies, as well as human clinical trials have pointed out that Ginseng extracts have negligible side effects (Park K. et al., 2014). Few unfavorable symptoms were reported following long periods of administration of high doses of Ginseng extracts. This included morning diarrhea, skin eruption, nervousness, sleeplessness, hypertension, edema, decreased appetite, depression, and hypotension (Siegel, 1979; Kiefer and Pantuso, 2003). A systematic review on PGEs in randomized controlled trials highlighted the safety of Ginseng. The review identified 40 studies where adverse effects were reported, but analysis revealed that out of the 40 studies, 16 studies showed no adverse events and 24 studies had 135 minor events (Shergis et al., 2013). Lee et al. reported that $P$. ginseng extract ( 1 or $2 \mathrm{~g} /$ day) supplemented over the course of 4 -weeks was safe, tolerable, and free of toxic effects in healthy volunteer subjects. Only non-significant changes were observed in hematological and biochemical tests (Lee et al., 2012). Recently, Song et al. performed a large-scale clinical study with 1000 participants randomly divided into two groups; a placebo and a group supplemented with $2 \mathrm{~g} / \mathrm{d}$ of KRG. Their findings asserted the safety and tolerability of KRG (Song et al., 2018).
Along the same lines, the mutagenic and toxicity potentials of tissue cultured mountain Ginseng adventitious roots (TCMGARs) were tested. TCMGARs did not exhibit any mutagenic properties when tested in diverse strains of Salmonella typhimurium and Escherichia coli. This was further shown in vivo without any evidence of TCMGARs mutagenicity, such as chromosomal aberration and micronucleus appearance, in mice exposed to TCMGARs (Murthy et al., 2018). All these studies confirm the biosafety and non-toxicity of Ginseng at an average dietary consumption.

Ginseng supplements have also shown certain clinically relevant patterns of adverse cardiovascular reactions. There are reports of numerous cases where prolonged Ginseng use or misuse has led to potential side effects related to cardiovascular events such as increased BP (Coon and Ernst, 2002), long QT syndrome, or atrial fibrillation (AF) (Paik and Lee, 2015). For example, in a young man, 3-year Ginseng supplementation has been found to correlate with hypertension, shortness of breath, dizziness, and inability to concentrate, symptomatology that disappeared and did not recur after stopping the supplements. In another instance, a hypertensive female receiving no other medication than Ginseng (Ginzin tablets; Ferrosan) reported an increase in BP rather than a decrease. Interestingly, such Ginseng-associated BP increase remitted going back to pretreatment levels 4 days after the cessation of Ginseng intake (Coon and Ernst, 2002). Although the observed effects appeared not to be clinically relevant, in a 30-subjects prospective, randomized, double-blind, placebo-controlled study, Ginseng was found to prolong the QTc interval and reduce DSB in healthy adults as early as $2 \mathrm{~h}$ after consumption (Caron et al., 2002). A 43-year-old healthy woman without familial history of sudden cardiac death and negative test of long QT mutations developed a long QT syndrome followed by polymorphic ventricular tachycardia. The woman admitted to the hospital revealed she was consuming $70 \mathrm{cL}$ of caffeine and $4 \mathrm{~L}$ of Korean Panax ginseng daily for 6 months. Upon stopping Ginseng consumption, the patient had no subsequent events. Yet, it is not proven whether a higher dose of Ginseng or a synergistic effect of caffeine could further prolong QT leading to malignant dysrhythmias (Torbey et al., 2011). Additionally, an AF with slow ventricular rate developed after taking AG for 1-week in an 83-year old woman with chronic renal disease (Liao et al., 2010). Nevertheless, all these mentioned episodes are considered rare adverse reactions that mostly depend on inter-variability between patients (Paik and Lee, 2015).

Ginseng has been reported to interact with several drugs, yet its interaction with warfarin (blood-thinning drug) is the most documented (Yuan et al., 2004; Chua et al., 2015). A randomized, double-blind, placebo-controlled trial using 20 healthy patients concluded that a 2-week intake of American Ginseng (2 g/d; $1 \mathrm{~g}$ twice daily) significantly reduced peak international normalized ratio (INR) and peak plasma warfarin levels (Yuan et al., 2004). In a recent study performed on rats, ginsenosides were reported to significantly enhance the activity of two enzymes known to metabolize warfarin, P450 CYP3A4 and P450 CYP2C9, restoring the levels of coagulation factors II and VII and that of the protein 
Z, that are usually suppressed by warfarin (Dong et al., 2017). The combined use of Panax ginseng with the monoamine oxidase inhibitor, phenelzine (Nardil), may result in manic-like symptoms (Vogler et al., 1999). Finally, although the efficacy and safety of Ginseng has been evidenced in numerous clinical studies, additional well-designed, large-scale randomized control trials are needed.

\section{GINKGO BILOBA}

Ginkgo biloba, also known as the maidenhair tree in English due to its resemblance to the foliage of the Maidenhair fern (Figure 3), is among the oldest seed plants. It is regarded as a "living fossil" because of its continued existence without dramatic changes for 270 million years. (Hori et al., 1997). Its place of origin is believed to be eastern China in Yangtze River Valley (Jaggy and Koch, 1997; Singh et al., 2008). From there, it became extensively distributed in Asia, Europe, North America, and New Zealand and is now widely cultivated (Kleijnen and Knipschild, 1992; Hori et al., 1997; Belwal et al., 2019). A remark about its leaf extract is included in the medical Dictionary of the Republic of China (Kimbel, 1992; Kleijnen and Knipschild, 1992; Kressmann et al., 2002). Ginkgo biloba is the only living species of the division Ginkgophyta probably due to its resistance to environmental stresses (Deng et al., 2006; Cao et al., 2012).

Ginkgo biloba is one of the most sold medicinal plants. It is one of the herbs mentioned in the Chinese Materia Medica more than 5000 years ago, where its seeds and leaves - fresh or driedhave been used for thousands of years in ancient herbal medicine. Current research on its therapeutic properties mainly uses Ginkgo biloba leaves and many pharmaceutical companies including those in the USA and Europe manufacture and sell extracts of the leaves (Kimbel, 1992; Kleijnen and Knipschild, 1992; Kressmann et al., 2002). The leaves can be used for the treatment of asthma and bronchitis in the form of a tea that is most commonly used by the Chinese people. More commonly, a standardized extract containing the most active constituents can be made from the leaves and then taken as a tablet, in liquid form, or given intravenously (Kleijnen and Knipschild, 1992).

The main constituents of Gingko biloba are flavonoids (ginkgo-flavone glycosides), terpenoids (ginkgolides and bilobalides), biflavones, and organic acids among other substances (Figure 3). Ginkgolides, being unique to Gingko biloba, are not synthesized by any other living species. Ginkgolides are classified into either A, B, C, J, or M types (Figure 3). Gingko biloba flavonoids include several

A

B

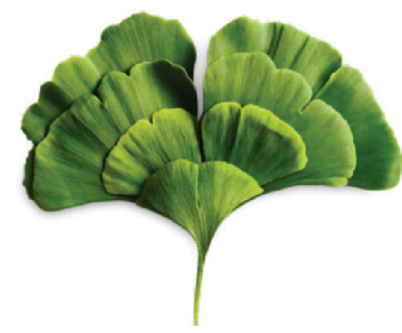

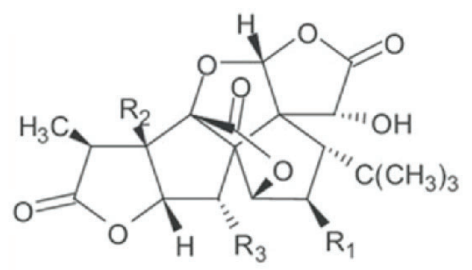

\begin{tabular}{lccc}
\hline & R1 & R2 & R3 \\
\hline Ginkgolide A & H & OH & H \\
\hline Ginkgolide B & H & OH & OH \\
\hline Ginkgolide C & OH & OH & OH \\
\hline Ginkgolide J & OH & OH & H \\
\hline Ginkgolide M & OH & H & OH \\
\hline
\end{tabular}

C

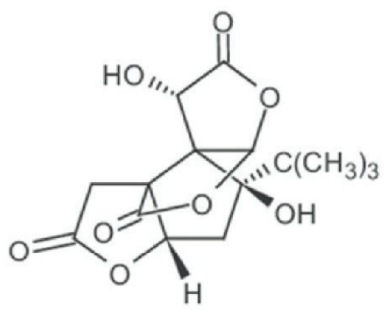

D<smiles>[R2]Oc1c(-c2cc([R3])c(O)c([R4])c2)oc2cc(O)c([R])c(O)c2c1=O</smiles>

FIGURE 3 | Ginkgo biloba. (A) Leaves of Ginkgo biloba or Maiden Hair Tree (from https://pngtree.com/freepng). (B) Chemical structure of Ginkgolides. (C) Chemical structure of Bilobalides. (D) Structural skeleton of flavonoids. R1 and R2 are side chains. 
representative glycosides, such as kaempferol, quercetin, and isorhamnetin (Figure 3). Flavonoids are known to reduce free radical generation and terpenoids are known to reduce inflammation and protect nerve cells against neuroinflammation (Kleijnen and Knipschild, 1992; Ude et al., 2013; Isah, 2015). Through a multistep process, Ginkgo biloba dried leaves extracts are enriched for flavonoids and terpenoids and the unwanted substances are eliminated. At the final step, the liquid extract is dried to give 1 part extract from 50 parts of raw drug (leaves) (Kleijnen and Knipschild, 1992; Isah, 2015). The composition of Gingko biloba extracts may differ depending on the manufacturing process. Standardized extract forms have been developed and usually contain $24-36 \%$ flavone glycosides and $4-11 \%$ terpenoids. For example, standardized extract EGb761 is the most commonly used Ginkgo biloba extract (GBE), and it contains $24 \%$ ginkgo flavonoid glycosides, $6 \%$ terpene lactones, and 5-10\% organic acids (Kressmann et al., 2002; Chan et al., 2007). These extracts have been used for various therapeutic purposes, including regulation of cerebral blood flow (Mashayekh et al., 2011), protection against free radicals (Oyama et al., 1996; Bridi et al., 2001), tinnitus treatment (Mahmoudian-Sani et al., 2017), protection of neurons (Mahdy et al., 2011), as well as enhancement of cognitive functions, such as memory and concentration problems (Weinmann et al., 2010; Tan et al., 2015).

\section{Ginkgo biloba at the Bench: Mechanism of Action in CVDs}

Ginkgo biloba's therapeutic effects and pharmacological actions are majorly due to its constituent flavonoids (ginkgo-flavone glycosides) and terpenoids (ginkgolides and bilobalide) (Lacour et al., 1991). These Ginkgo biloba constituents are well known for their antioxidant and anti-inflammatory effects. Ginkgo biloba antioxidant and anti-inflammatory effects are beneficial in a plethora of diseases that include cardiovascular, pulmonary, and central nervous systems.

Free radical generation contributes to the development and progression of numerous CVDs, including vascular injuries and atherosclerotic plaque formation. During CVD pathogenesis, the equilibrium between free radical generation and antioxidant defense is greatly shifted toward the former (Singh and Niaz, 1996; Witztum and Berliner, 1998; Fulton and Barman, 2016). GBE greatly restores the disturbed oxidative state equilibrium due to their antioxidant action, which helps to scavenge excessive free radicals as well as reduce free radical generation.

In addition, vasodilatory and antihypertensive properties of GBE can exert cardioprotective benefits (Perez-Vizcaino et al., 2009). In this regard, GBE has exhibited ACE inhibitory activities (Mansour et al., 2011), activation of cholinergic pathways, endothelial health improvement, inhibition of endothelium activation and adhesion (Mesquita et al., 2017), and serum lipid-lowering activities (Liou et al., 2015; Huang et al., 2018) among other reported effects that are beneficial in CVD.

By acting as an anti-atherothrombotic and anti-inflammatory agent, GBE can limit LPS-induced proliferation of VSMCs and their morphological alterations. Furthermore, GBE can regulate the inflammatory response in blood vessels by decreasing the activity of the ROS producing enzyme, nicotinamide adenine dinucleotide phosphate (NADPH) oxidase (NOX), and reducing the phosphorylation of mitogen-activated protein kinases (MAPKs). Subsequently, MAPKs suppress toll-like-receptor-4 (TLR-4) expression in human aortic smooth muscle cells (Lin et al., 2007). GBE can also decrease the production of the enzyme involved in the rupture of atherosclerotic plaques, MMP-1, in oxidized LDL- and 4-hydroxynonenal-induced human coronary smooth muscle cells (Akiba et al., 2007). In the same model, the GBE constituent, Ginkgolide B, attenuated endothelial dysfunction by inhibiting monocyte chemotactic protein-1 (MCP-1), intercellular adhesion molecule-1 (ICAM-1), and vascular cell adhesion molecule-1 (VCAM-1) production in oxidized-LDL-induced HUVECs. Additionally, Ginkgolide B treatment reduced the expression of several inflammatory cytokines in oxidized-LDL-induced mouse RAW264.7 macrophages (Feng et al., 2018). Ginkgolide C, another GBE constituent, can reduce adipogenesis and enhance lipolysis leading to suppression of lipid accumulation. Ginkgolide C treatment of 3T3-L1 adipocytes decreased the expression of PPAR adipogenesis-related transcription factors. Ginkgolide C also enhanced the Sirt1/AMPK pathway resulting in decreased activity of acetyl-CoA carboxylase and fatty acid synthesis. Moreover, Ginkgolide C stimulated the production of adipose triglyceride lipase and hormone-sensitive lipase, leading to elevated lipolysis levels (Liou et al., 2015). Similar results were obtained with human HepG2 hepatocyte cell line (Huang et al., 2018).

\section{In Vivo Preclinical Evaluation of Ginkgo biloba}

Ginkgo biloba has several cardioprotective effects, including improvement of atherosclerosis due to their ability to block platelet-activating factor and platelet aggregation in rats (Zeng et al., 2013; Huang et al., 2014).

eNOS is responsible for most of the vascular NO production, and $\mathrm{NO}$ acts as a protective molecule to maintain vasculature hemostasis and protection of the vascular endothelium (Forstermann and Munzel, 2006). eNOS production and activity are impaired in several CVDs, including hypertension (Chou et al., 1998), cardiac hypertrophy (Ozaki et al., 2002), myocardial infarction (Tsutsui et al., 2008), and heart failure (Couto et al., 2015). GBE can act as an antihypertrophic agent by the activation of the $\mathrm{M}_{2}$ muscarinic receptors/NO pathway and of cholinergic signaling during cardiac hypertrophy. In a rat model of chronic $\beta$-adrenergic stimulation-induced cardiac hypertrophy, GBE was able to ameliorate the deleterious cardiac events associated with cardiac hypertrophy. These effects were mediated by the upregulation of $M_{2}$ receptors and the downregulation of $\beta_{1^{-}}$ adrenergic receptors. GBE also restored eNOS activity and consequently elevated NO levels (Mesquita et al., 2017). In addition, the anti-hypertensive effects of EGb761 supplementation were documented in hypertensive rats where SBP, DBP, and arterial BP were reduced. EGb761 supplementation also decreased inflammation and oxidative stress. While eNOS protein 
expression levels were enhanced, protein levels of iNOS were decreased (Abdel-Zaher et al., 2018).

Vascular aging is commonly accompanied with low-grade inflammation and degenerative structural changes and stiffness of blood vessels and is considered a risk factor for the development of CVDs, such as CHD and hypertension (Franceschi et al., 2000; Lakatta and Levy, 2003). In the mesenteric arterioles of old rats, GBE had a protective effect that alleviated arterial stiffness and improved endothelial health (Cuong et al., 2019). In these aged mesenteric arterioles, GBE improved vascular elasticity by narrowing the EC gap, increasing curvature of inner elastic membrane and reducing the middle collagen fiber layer. These changes were accompanied by decreased phosphorylation levels of Akt/FoxO3a signaling components, which usually contributes to vascular dysfunction (Cuong et al., 2019).

Pre-treatment with EGb761 in rats that have undergone myocardial ischemia-reperfusion injury inhibited the apoptosis of myocardial cells, decreased the expression of caspase 3 and proapoptotic Bax and increased that of anti-apoptotic Bcl-2, and protected the myocardium by activating the endogenous Akt/Nrf2 antioxidant stress pathway. Akt/Nrf2 activation subsequently decreased oxidative stress leading to reduced lipid peroxidation and increased activities of the endogenous anti-oxidant defense enzymes, namely SOD, and GSH-Px. In addition, EGb761 pretreatment increased the expression of the heat shock protein heme oxygenase 1 (HO-1) and repressed the expression of mediators of the inflammatory response, such as TNF- $\alpha$, IL- 6 , and IL- $1 \beta$ (Chen et al., 2019). HO-1 degrades heme (a potent oxidant) to generate carbon monoxide, which has anti-inflammatory properties, bilirubin, which is an antioxidant derived from biliverdin, and iron (He et al., 2014). Similar Ginkgo biloba anti-oxidant properties have been reported in diabetic rats as well. Administration of GBE for 30 days can increase SOD, CAT, and GSH-Px activity along with glutathione (GSH) levels in the liver and pancreas of diabetic rats (Cheng et al., 2013). This enhanced antioxidant status might be responsible for improved glucose uptake via increased GLUT-4 expression (Shi et al., 2010). Furthermore, EGb761 oral supplementation of HFD-fed mice can dosedependently enhance glucose tolerance, decrease insulin levels, and diminish parameters of insulin resistance (Cong et al., 2011).

The above reports point that GBE has a pleiotropic mechanism of action. Indeed, a metabolomic profiling study of the plasma and hearts of GBE-supplemented rats with myocardial infarction established that GBE acts via the regulation of multiple metabolic pathways. Metabolomic profiles of rats with MI showed disturbed metabolism in these rats because of modulated inflammatory reaction, oxidative stress, and structurally damaged pathways. However, GBE supplementation controlled the inflammatory reaction and oxidative stress pathways by regulating sphingolipid, phospholipid and glyceride metabolism and ameliorated the structural damage by downregulating amino acid metabolism (downregulation of urea cycle) and decreasing oxidative stress (Wang et al., 2016).

In addition to the above-mentioned effects, GBE was able to decrease calcium overload (Liu et al., 2013), the primary factor responsible for the irreversible myocardial injury (Moens et al.,
2005). Rats with an ischemic myocardium and pre-treated with GBE50, an extract that matches EGb761, exhibited decreased intracellular calcium overload which could block arrhythmia. GBE could decrease the calcium overload and protect from an ischemic myocardium by inhibiting the $\mathrm{Na}^{+} / \mathrm{Ca}^{2+}$ exchanger (Liu et al., 2013).

\section{Ginkgo biloba to the Clinic}

Given the above reported protective and therapeutic benefits of GBE in vitro and in vivo, several clinical trials have been conducted to test different formulations and doses of GBE in a plethora of diseases (DeKosky et al., 2008; Gardner et al., 2008; Kuller et al., 2010; Hashiguchi et al., 2015). A search of clinicaltrials.gov shows that there have been 88 reported clinical trials using various formulation of GBE. Of the 88 trials, 66 have been concluded, and there are 30 Phase 3 or 4 trials. Most of these trials dealt with neural and cognitive disorders, where GBE has been shown to have clinical promise. For GBE beneficial effects in CVDs, 7 out of the 88 trials were concerned with vascular diseases, 4 with stroke, 4 with arteriosclerosis, 2 with coronary disease, 1 with hypertension, and 1 with atherosclerosis.

GBE has vasorelaxation effects in human subjects. GBE was able to dilate forearm blood vessels causing changes in regional blood flow without affecting BP levels in 16 healthy subjects (Mehlsen et al., 2002). A small trial performed in normal glucose-tolerant subjects to determine the effects of GBE on glucose-stimulated pancreatic beta-cell function found that the ingestion of GBE for three months can decrease SBP and DBP. In these individuals, fasting plasma insulin and CRP were increased (Kudolo, 2000). A double-blind, placebo-controlled, parallel design trial was performed in patients with peripheral artery disease aimed to assess the effects of the supplementation of 300 $\mathrm{mg} /$ day of EGb761 to treadmill walking time and cardiovascular measures. In older adult patients, EGb761 produced a modest non-significant increase in maximal treadmill walking time and flow-mediated vasodilation. The authors suggested that a longer duration might be needed to observe significant beneficial effects (Gardner et al., 2008).

Kuller el al. used the Ginkgo Evaluation of Memory Study (GEM) to assess CVD as a secondary outcome. The GEM study was a double-blind trial that randomized 3069 participants whose ages were over 75 years to $120 \mathrm{mg}$ of EGb761 twice daily (240 mg/day) or placebo. Data indicated that EGb761 did not affect the originally assessed primary outcome-the development of dementia or Alzheimer's disease (DeKosky et al., 2008). Also, there were no differences in the incidence of myocardial infarction, angina pectoris, or stroke between the GBE and placebo groups. After 6 years of monitoring, the study concluded that GBE does not reduce total CVD mortality or CVD events (Kuller et al., 2010).

Several clinical trials to assess the protective effects of GBE in CVDs are still ongoing. A 12-weeks randomized, double-blind, phase 3 clinical trial aimed to further evaluate the safety and efficacy of Rinexin ${ }^{\circledR}$ (Cilostazol 100mg, Ginkgo biloba leaf extract $80 \mathrm{mg}$ ) which is widely used as an anti-platelet agent for the treatment of 
peripheral artery disease (NCT03318276; clinicaltrials.gov). Most recently, efficacy and safety of Ginkgo biloba pills for CHD patients with impaired glucose regulation will be assessed in a Phase 4 randomized, double-blind, placebo-controlled clinical trial (NCT03483779; clinicaltrials.gov). Twelve patients will be recruited for a test period of 58 weeks. Pills of five different GBEs will be to administered three times a day (Sun M. et al., 2018).

The therapeutic effect GBE appears to be more evident in combination with modern medicine. The analysis of 23 randomized clinical trials (involving 2,529 patients) showed that when combined with routine Western medicine, GBE was more effective at the relief of angina pectoris as compared to the routine medicine alone (Sun et al., 2015). In addition, due to its platelet aggregation inhibitory effects, the combination of GBE and modern medicine was reported to posses beneficial effects against acute cerebral ischemia. In that study, platelet aggregation was found to be significantly lower in patients treated with ticlopidine and EGb 761 as compared with patients treated with ticlopidine alone (Hong et al., 2013). Combination of EGb 761 also had increased therapeutic effect in patients with uncontrolled diabetes. Indeed, a randomized controlled trial showed that the combination of EGb 761 with metformin is more effective than metformin alone in improving the outcomes of patients with uncontrolled T2DM (Aziz et al., 2018).

The GBE therapeutic potential in managing CVDs has not been always clinically observed. Using data obtained from the GEM study database, Brinkley et al. concluded that GBE does not reduce $\mathrm{BP}$ or the incidence of hypertension in older men and women (Brinkley et al., 2010). In accordance, another study reporting the analysis of 9 randomized clinical trials (1012 hypertensive patients) concluded that more rigorous trials are needed to draw a conclusion on the efficacy of GBE in managing hypertension (Xiong et al., 2014).

Based on these and other studies, the efficacy of GBE, despite being reported in many studies, is best documented and observed when combined with other known medications for the management of CVDs.

\section{Safety, Toxicity, and Side Effects of G. biloba}

Taken orally at the typical dosage, GBE may cause mild adverse effects, principal among which are mild gastrointestinal upset, headache, dizziness, constipation, and allergic skin reactions. Higher dosages, however, can result in restlessness, diarrhea, nausea, vomiting, and weakness (Diamond and Bailey, 2013). Noticeably, the therapeutic employment of this herb is also linked to adverse cardiovascular events. Fifteen published case reports described a temporal association between GBE intake and serious bleeding events, including intracranial bleeding (Bent et al., 2005), an effect that may be attributed to the platelet-activating factor antagonism exerted by ginkgolides, bilobalides, and other constituents present in the extract (Izzo and Ernst, 2009). These major bleeding events, including subarachnoid and intracranial hemorrhage, have been mostly described during the concomitant use of gingko and antiplatelet and/or anticoagulant medications (Matthews, 1998). Therefore, it is recommendable to stop GBE intake at least 2 weeks before surgical procedures. Always because of its anti-platelet properties, it has been suggested that GBEs (including seeds and leaves) should be used with caution during pregnancy, particularly around labor, and during lactation (Dugoua et al., 2006). Several case reports described cardiac adverse events associated with Ginkgo biloba leaf extracts. For example, 2 weeks GBE intake ( $40 \mathrm{mg}$, three times daily) has been reported to develop ventricular arrhythmia in a 49 -year-old subject with good health (Cianfrocca et al., 2002), a symptom resolved upon the discontinuation of GBE supplementation (Cianfrocca et al., 2002).

A randomized placebo-controlled, double-blind pilot study of GBE reported more ischemic stroke and transient ischemic attack cases in the GBE group as compared with the placebo. The study lasted 42-month 118 cognitively intact subjects randomized to standardized GBE or placebo and its aim was to measure the effect of GBE on cognitive decline (Dodge et al., 2008). Another case report, attributed the frequent nocturnal palpitations reported by a 35-year old woman taking GBE supplementation to GBE (Russo et al., 2011). In addition to clinical trials, Ginkgo biloba safety has also been assessed in vivo in rats. Dietary intake of GBE ( $0.5 \%$ extract) for 4 weeks has been reported to significantly reduce heart rate and blood flow velocity in tail arteries of old spontaneously hypertensive (SH) rats as compared to the control group (Tada et al., 2008). Thus, in the elderly population with hypertension, the use of GBE may need to be assessed for effects on heart rate (Mei et al., 2017).

Furthermore, some of the components (ginkgolic acids) of EGb761 have been reported to elicit severe allergic reactions. However, this allergic reaction is not present as long as the carboxylic acid group of ginkgolic acids is intact (Chan et al., 2007). Yet, contact with Ginkgo biloba plants is associated with severe allergic reactions, including erythema and edema (Chiu et al., 2002)

Food poisoning by Ginkgo biloba seeds has been reported in Japan and China, where the main symptoms were convulsion, vomiting, and loss of consciousness. The poisoning is primarily due to the neurotoxic compound 4'-O-methylpyridoxine (MPN, also known as ginkgotoxin) which interferes with pyridoxine (vitamin $\mathrm{B}_{6}$ ) metabolism, leading to serious neurological manifestations including neurotoxicity, seizures, and loss of consciousness (Wada et al., 1988; Wada, 2005). Ginkgotoxin is found in the ginkgo leaf at very low amounts. However, GBE is unlikely to contain this toxic component as ginkgotoxin is standardized to be too low in the extract (Arenz et al., 1996).

Several reports have described that GBE induces cytochrome P450 (CYP) in humans, shedding light on potential interactions between GBE and conventional drugs. Ginkgo biloba is known to decrease the plasma concentrations of omeprazole, ritonavir, and tolbutamide. It can interact with antiepileptics, acetylsalicylic acid, diuretics, ibuprofen, risperidone, rofecoxib, trazodone, and warfarin (Izzo and Ernst, 2009).

Considering that GBE is widely used in a plethora of diseases combined with the paucity of data from animal studies regarding 
GBE toxicity and carcinogenicity, the National Institutes of Health (NIH) has performed a 2-year and 3-month toxicity and carcinogenicity study of GBE in B6C3F1/N mice and F344/N rats using different doses of GBE. The GBE used contained 24\% flavonol glycosides and $6 \%$ terpene lactones, along with no more than five ppm ginkgolic acids. The study was performed by NIH National Toxicology Program (NTP) and concluded that GBE might elicit toxic and cancer-related consequences in rodents. The carcinogenic effects reported were stomach ulcers, organ modification including carcinogenic activity in the liver, liver and thyroid gland hypertrophy, liver hyperplasia, and hyperkeratosis (National Toxicology Program, 2013; Rider et al., 2014). These reports raised concerns about the safety of GBE. Following the NTP report, the International Agency for Research on Cancer (IARC) reported in 2014 that there is inadequate evidence in humans for the carcinogenicity of GBE (Grosse et al., 2013). Following this report, clinical and genomic safety of IDN 5933/ Ginkgoselect ${ }^{\circledR}$ Plus, a standardized GBE, was assessed in elderly subjects using a randomized placebo-controlled clinical trial. The treatment group was given 120-mg IDN 5933 twice-daily for 6 months. No adverse clinical effects or increase of liver injury markers were reported in the treatment group. Genomic testing revealed that there is no difference in micronucleus frequency or DNA breaks between the treated and placebo groups. The expression of genes known to be modulated in early carcinogenesis (c-myb, $p 53$, and ctnnb1 [ $\beta$-catenin]) was not significantly different between groups at the beginning or the end of the study (Bonassi et al., 2018). Taken together these results support the safety of IDN 5933 at the used doses for a duration of 6 months. Overall, there is still controversy about the safety of GBE for long-term use in human subjects and additional well- designed clinical trials that assess the safety and efficacy of GBE are much needed.

\section{GANODERMA LUCIDUM}

Ganoderma lucidum, also known as "lingzhi" or "reishi," is a mushroom (Figure 4) whose different parts (mycelia, spores, and fruit body) are used to make different forms of commercial $G$. Lucidum for their medicinal benefits. Commercially, G. Lucidum is available as powders, dietary supplements, tea, among other forms. Historically, G. Lucidum medicinal use has been wide spread in Asian countries (mainly in China, Japan, and Korea) for more than 2000 years. Later, it was introduced to Western societies (Ahmad, 2018). Hot water or ethanol can be used to extract the bioactive compounds from the fruiting bodies, the mycelia, or the spores of the mushroom (Heleno et al., 2012). A wide array of bioactive compounds exist in G. Lucidum that include triterpenes (Figure 4), polysaccharides, nucleosides, steroids, fatty acids, alkaloids, proteins, peptides, amino acids, and inorganic elements (Ahmad, 2018).

Immunomodulation, anti-oxidation, liver protection, antiproliferation, and anti-angiogenesis are among the various properties that Ganoderma lucidum bioactive compounds possess individually or synergistically (Sanodiya et al., 2009). The triterpenoids have hepatoprotective, anti-hypertensive, hypo-cholesterolemic, anti-histaminic, anti-tumor, and antiangiogenic effects. In addition, Ganoderma lucidum triterpenoids have anti-platelet aggregation and complement inhibition effects. It should be noted that G. Lucidum is the only source of triterpene fatty acids called ganoderic acids. Of the 200 bioactive compounds that have been identified in Ganoderma lucidum extracts,

A

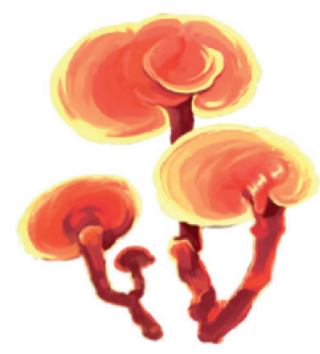

B

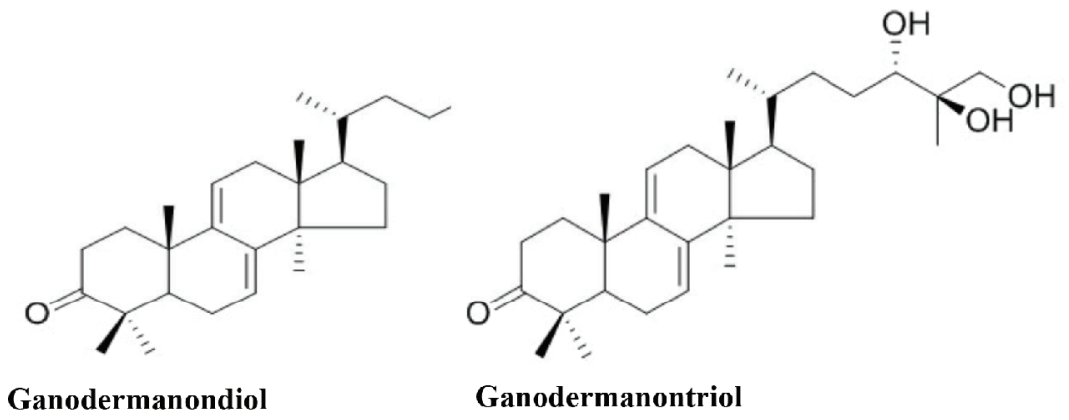

FIGURE 4 | Ganoderma lucidum. (A) Ganoderma lucidum (from https://pngtree.com/freepng). (B) Examples of the chemical structure of two Triterpenes from Ganoderma lucidum. 
ganoderic acids $\mathrm{A}, \mathrm{B}$, and $\mathrm{C}$ have hypoglycemic effects (Hikino et al., 1985; Tomoda et al., 1986), while ganoderic acids F, B, D, H, $\mathrm{K}, \mathrm{S}$, and Y most likely have hypotensive effects (Morigiwa et al., 1986). Ganoderma lucidum polysaccharides ( $\beta$-d-glucans) harbor anti-tumor properties due to their immunomodulation and antiangiogenesis effects. They also exhibit anti-oxidant protective effects against free radicals and can decrease mutagen induced cell damage (Boh et al., 2007). Ganoderma lucidum $\beta$-d-Glucan polysaccharides were also identified as the active component in the polysaccharide peptide (PsP) usually found in Ganoderma lucidum extracts. There is no agreed dosage for G. lucidum, however, commonly used doses vary between 1.5 and $9 \mathrm{~g}$ of dried extract per day (Klupp et al., 2016).

\section{G. lucidum at the Bench: Mechanism of Action in CVDs}

As stated earlier, antioxidants are very important when it comes to the prevention of atherosclerosis. G. lucidum can act as an antioxidant; in the model organism Caenorhabditis elegans, $G$. lucidum protected C. elegans against paraquat and heavy metalinduced oxidative stress via the diet restriction pathway and the mTOR/S6K signaling pathway, respectively (Cuong et al., 2019). G. lucidum has been found to protect human lymphocyte DNA from hydrogen peroxide-induced oxidative damage (Shi et al., 2002). It was also found that G. lucidum regulates the expression of Nrf2 which in turn regulates antioxidants genes such as HO-1, GST, NQO-1; G. lucidum ethanol extract enhanced the phosphorylation of Nrf2 which upregulated HO-1 in C2C12 myoblasts (Lee Y. et al., 2016). Thus, the antioxidant power in G. lucidum, which is important for the prevention of atherosclerosis, can act on different signaling pathways.

Elevated BP can be detrimental to heart function. Three peptides (QLVP, QDVL, and QLDL), that can inhibit ACE activity and called ACE inhibitory peptides (ACEIPs), were extracted from $G$. lucidum and can be used to treat hypertension. QLVP can inhibit ACE by its interaction with Gln242 and Lys472 of ACE. QLVP also enhanced Angiotensin 1-mediated phosphorylation of eNOS and reduced mRNA and protein expression of the vasoconstrictor peptide endothelin-1 in HUVECs (Wu et al., 2019).

Selenium-enriched G. lucidum polysaccharide (Se-GLP) extracts have protective effects against oxidative damage in a mouse model of heart reperfusion injury. Se-GLP significantly reduced the ischemic reperfusion injury-induced serum levels of MDA as well as the levels of the proinflammatory molecule intercellular adhesion molecule-1. Heart and serum levels of the antioxidant enzymes SOD, CAT, and GSH-Px and the levels of GSH as well as total antioxidant capacity were rescued by Se-GLP (Shi et al., 2010). In another study, the protective effects of Ganoderic acid A, from $G$. lucidum extracts, was found to activate the PI3K/AKT signaling pathway causing increased proliferation and decreased apoptosis of rat $\mathrm{H} 9 \mathrm{c} 2$ cardiomyocytes exposed to hypoxic injury, a phenomenon mediated by the activation of miR-182-5p and reduction of PTEN expression (Zhang et al., 2018). Similarly, a preclinical study using transverse aortic constriction mice as a model of pressure overloadcaused cardiomyopathy reported improved cardiac function following treatment with spore oil extracted from G. Lucidum (Xie et al., 2016).

More research needs to be conducted on the possible mechanisms and signaling pathways that G. Lucidum bioactive derivatives employ to elicit their beneficial effects in order to get a more comprehensive sense of how these substances work.

\section{In Vivo Preclinical Evaluation of G. Iucidum}

To study the cardioprotective effects of G. lucidum extract, global ischemia and reperfusion of isolated and perfused rat hearts were used. As a preventative treatment, $400 \mathrm{mg} / \mathrm{kg}$ body weight of $G$. lucidum extract was delivered to rats for 15 days. It was found that this treatment halted the necrotic death of the rat cardiomyocytes and reduced the reperfusion contracture (Lasukova et al., 2015). Interestingly, G. lucidum extract supplementation attenuated diastolic dysfunction and prevented irreversible cardiomyocyte damage (Lasukova et al., 2008).

Diabetes mellitus is a metabolic disease that has been correlated with a high incidence of CVDs, partly because high blood glucose levels can cause vascular damage (Klein et al., 2002; Fox et al., 2008; Shah et al., 2008). Thus, a supplement that can help manage diabetes would be of great benefit to decrease CVD risk. Thirty-five Wistar rats were supplemented with 50, 150 , and $300 \mathrm{mg} / \mathrm{kg}$ of PsP from G. lucidum extracts. PsP induced endothelial repair process with $300 \mathrm{mg} / \mathrm{kg}$ being the most efficient dose. The findings showed that vascular damage was improved by PsP treatment in a rat model of T2DM (Heriansyah et al., 2019).

A diet high in fats is known to be a contributing risk factor to the development of CVDs (Fleming, 2002) and weight loss is an essential measure for the prevention of CVDs in obese people (Sowers, 2003). The water extract of G. lucidum can reduce the body weight, inflammation, and insulin resistance in HFD-fed mice (Chang et al., 2015). In another study, G. lucidum spores (GLSP) at a dose of $1 \mathrm{~g}$ /day supplemented orally for 4 weeks were used in adult male Sprague-Dawley rats. GLSP decreased total cholesterol and triglycerides in diabetic rats as well as attenuated the levels of oxidative stress; it is worth noting that there was an upregulation of genes related to lipid metabolism-acyl-CoA oxidase 1 (ACOX1), acetyl-CoA carboxylase (ACC), and Insig-1/ 2 gene expression. ACOX1 was activated more than 5 -folds in the GLSP-treated diabetic rats which indicates an accelerated beta-oxidation of lipids in these rats (Wang et al., 2015).

Dyslipidemia is one of the major risk factors for CVDs (Miller, 2009). In experimental animal studies, G. lucidum showed anti-hyperlipidemic effects by lowering plasma total cholesterol, LDL, and triglyceride levels (Chen et al., 2005). Hydrogen peroxide free radicals are usually elevated during dyslipidemia, and thus the risk of atherosclerosis, so antioxidants are needed to prevent blood vessels damage. A study conducted in high cholesterol diet-supplemented Wistar rats that received 50,150, and $300 \mathrm{mg} / \mathrm{kg}$ bodyweight of PsP derived from G. Lucidum found that PsP acted as a potent antioxidant. In addition, PsP may prevent the atherogenesis process in the context of dyslipidemia with the optimum dose being $300 \mathrm{mg} / \mathrm{kg}$ bodyweight (Wihastuti and Heriansyah, 2017). 
Another study found that polysaccharides extracted from $G$. Lucidum significantly reduced the body-weight increases of mice fed an HFD suggesting its role as a hypolipidemic substance. In addition, it exhibited antioxidant and antiapoptotic effects in the HFD-fed mice (Liang et al., 2018).

With regard to regulation of high $\mathrm{BP}$, a study conducted on adult male hypertensive rats that received G. lucidum water extracts intra-gastrically for seven weeks found that the experimental rats $\mathrm{BP}$ was reduced to a level comparable to that of rats on losartan (angiotensin II receptor antagonist) (Shevelev et al., 2018)

\section{G. Iucidum to the Clinic}

Antioxidants are potentially therapeutic substances that can be used to prevent atherosclerosis as well as a variety of other diseases. Several in vitro and in vivo preclinical studies have shown that G. lucidum constituents possess antioxidant activities, but evidence for such activities in human subjects was lacking. Watchtel Galor et al. performed a follow-up study on the effects of G. lucidum supplementation on an array of parameters that included antioxidant biomarkers status and CHD risk. A double-blinded, crossover, placebo-controlled intervention study of 18 healthy people was conducted. There was an enhancement of plasma total antioxidant markers status as well as an improvement of CHD biomarkers after 10 days of supplementation. More importantly, there was no renal, liver, or DNA toxicity (Wachtel-Galor et al., 2004). To test G. Lucidum antioxidant potential, a crossover human intervention study was conducted on seven healthy people, and it was found that the plasma total antioxidant power was enhanced after the administration of a single dose of G. Lucidum extract. Lymphocytes harvested following blood collection, however, showed no signs of enhanced DNA repair (Wachtel-Galor et al., 2010). G. lucidum PsP was also examined for its antioxidant properties. A clinical trial was conducted with 37 high risk and 34 stable angina patients that were given PsP $750 \mathrm{mg} /$ day for 90 days. PsP proved to be a potent antioxidant in the context of atherosclerosis in both the high angina risk and stable groups. PsP supplemented groups showed increased SOD, decreased MDA levels, and reduced counts of circulating endothelial cells (CEC) and endothelial progenitor cells (EPC) (Sargowo et al., 2018).

A double-blind, randomized, multicentered study was done to evaluate the effects and safety of G. lucidum polysaccharides on patients with CHD. Eighty-eight patients that constituted the experimental group were given G. lucidum polysaccharides for 12 weeks. The polysaccharides significantly enhanced the wellbeing of the patients evidenced as an improvement of their major symptoms (chest pain, shortness of breath, palpitations), and a decrease in BP and serum cholesterol levels (Gao et al., 2004). Another study found that PsP from G. lucidum had antiinflammatory effects and vascular EC protection in patients with ST-elevation myocardial infarction and non-ST-elevation myocardial infarction with risk factors of dyslipidemia (Sargowo et al., 2019).
A randomized clinical trial was conducted on the use of $G$. lucidum for the treatment of cardiovascular risk factors of the metabolic syndrome. It was reported that using G. lucidum (3 g/ d) for 16 weeks had no effect on glycosylated hemoglobin (HbA1c) and fasting plasma glucose (FPG). Further, consumption of G. lucidum was associated with the increased risk of a subset of mild events including headache, fatigue, and gastrointestinal events (Klupp et al., 2016).

None of the human studies reported above had any serious side effects thus highlighting G. lucidum as a potential therapeutic and preventative substance in the context of certain CVDs.

In a study examining 37 patients with high risk for atherosclerosis and on an ongoing conventional CVD medication, G. lucidum PsP was able to reduce the levels hs-CRP, IL-6, and TNF- $\alpha$, as well as the levels of MDA (Widya et al., 2016). Another study involving 34 stable angina pectoris patients showed that administration of PsP in combination with previous medication is able to significantly reduce CECs and EPCs (markers for endothelial vascular injury) as well as the levels of total cholesterol (Ubaidillah et al., 2016).

The efficacy of G. lucidum in CVDs was tested in a prospective, double-blind, placebo-controlled trial. Eighty-four volunteers with T2DM and metabolic syndrome were supplemented with extracts of G. lucidum, G. lucidum with Cordyceps sinensis, or placebo in order to manage cardiovascular risk factors. Results from the study evidenced that G. lucidum failed to provide benefit against CVDs in patients with the metabolic syndrome (Klupp et al., 2016). Likewise, the analysis of five trials (398 total volunteers), concluded that $G$ lucidum was not effective in treating elevated BP (Klupp et al., 2015).

Taken together, these data suggest that G. lucidum is most effective in providing cardiovascular protection when combined with conventional CVDs medications.

\section{Safety, Toxicity, and Side Effects of G. Iucidum}

Numerous conducted studies and investigations pointed toward the safety of G. Lucidum. In both male and female rodents, $G$. lucidum shows no signs of toxicity even at a dose of up to 5000 $\mathrm{mg} / \mathrm{kg}$ of body weight. No animal mortality was reported either (Smina et al., 2011). When compared to doxorubicin (a typical DNA intercalating agent used in chemotherapy), G. lucidum extracts were shown to act indirectly on DNA, thus adding more assurance to its safety (Gurovic et al., 2018).

Hemostatic parameters, platelet and global hemostatic functions, were not affected when a dose of $1.5 \mathrm{~g} / \mathrm{d}$ of $G$. lucidum extract was given to healthy human volunteers for 4 weeks. In addition, the use of G. lucidum did not cause bleeding problems in the healthy subjects (Kwok et al., 2005). However, in another study, Tao and Feng volunteered 15 healthy subjects and 33 atherosclerotic patients and found that any dose above 3000 $\mathrm{mg} /$ day can inhibit platelet aggregation. As a result, caution is advised when supplementing G. lucidum to patients with low platelet count or patients that will undergo surgical procedures (Tao and Feng, 1990). 
Safety of polysaccharides extracted from the fruiting body of G. lucidum was evaluated in Wistar rats and the results indicated no evidence of abnormal clinical symptoms, death or significant differences in body weight and food intake. No significant differences were found in the hematology value, clinical chemistry value and organ/body weight ratio. Additionally, no mutagenicity was detected in Kunming mice (Zhang et al., 2016).

However, high doses of G. lucidum polysaccharides modulated immune responses. The polysaccharides enhanced the primary immune response to sheep red blood cells but did not have significant effects on the phagocytic function or macrophages (Zhang et al., 2016). The G. lucidum polysaccharides-modulated immune responses were tested in another study where 16 participants supplemented with $2 \mathrm{~g}$ of G. lucidum extract twice daily took part in a 10-day controlled trial. In this study, there were no differences in CD4, CD8, and CD19 levels in the blood, though CD56 did increase, without achieving statistical significance, but it returned to baseline levels 10 days after intake of the extract (Wicks et al., 2007).

A 12-week trial performed on 23 dyslipidemic and mild hypertensive volunteers revealed that Lingzhi $(1.44 \mathrm{~g} /$ day $)$ had no effect on several clinical chemistry parameters as compared with the placebo. However, symptoms such as headache, influenza/running nose were found, although considered not clinically significant (Chu et al., 2012).

Whether these changes that are induced by G. lucidum have any clinical value remains to be addressed in future clinical trials especially after long-term administration of escalating doses of $G$. lucidum extracts to assess long-term safety. Further studies are still needed to examine the toxicity, side effects, and safety of $G$. lucidum for human consumption.

\section{GYNOSTEMMA PENTAPHYLLUM}

Gynostemma pentaphyllum (Figure 5), also known as Jiaogulan, is a herbaceous climbing vine. Originating in south China and now widely distributed in South and East Asia (Li et al., 2016), it is found in subtropical China, Japan, Myanmar, and India (Chen and Gilbert, 2006), growing near rivers and in the shade of forests that surround Yangtze River basin and the southern areas of China (Chen, 1995). It can be found as a health supplement in beverages, biscuits, face washes, and bath oils (Li et al., 2016). The herb has a low level of genetic diversity and a high level of variation among populations (Zhang et al., 2019).

Phytochemical analysis found that G. pentaphyllum extracts contain gypenoside saponins, flavonoids, polysaccharides, and amino acids (Zheng, 2004; Nookabkaew et al., 2006; Yan et al., 2013). Its biological effects can range from antimicrobial (Srichana et al., 2011), antioxidant (Muller et al., 2012), anticancer (Schild et al., 2010), anti-inflammatory (Wong et al., 2017), antidiabetic (Yeo et al., 2008; Huyen et al., 2012), antilipidemic (la Cour et al., 1995), and neuroprotective (Keilhoff et al., 2017) to anti-obesity effects (Gauhar et al.,
2012; Park S. et al., 2014). It has been used to treat hepatitis and hypertension (Lin et al., 2000). Among the various bioactive compounds, dammarane-type triterpene saponins (gypenosides or gynosaponins) are major (Kim and Han, 2011).

\section{G. pentaphyllum at the Bench: Mechanism of Action in CVDs}

As already mentioned, antioxidants are important when it comes to the prevention of atherosclerosis. Four flavonoids-namely quercetin-3-O-(2", $6^{\prime \prime}$-di- $\alpha$-L-rhamnosyl)- $\beta$-D-galactopyranoside, quercetin-3-O-(2",6"-di- $\alpha$-L-rhamnosyl)- $\beta$-D-glucopyranoside, quercetin-3-O-(2" $\alpha$-L-rhamnosyl $)-\beta$-D-galactopyranoside, and quercetin-3-O-(2" $-\alpha$-L-rhamnosyl)- $\beta$-D-glucopyranoside-with potent antioxidant effects against $\mathrm{DPPH}$ and $\mathrm{OH}$ free radicals, in vitro, were found in the extracts of $G$. pentaphyllum. These flavonoids also exhibited cytoprotection against AAPH-induced oxidative damage in pig kidney LLC-PK1 cells by suppressing the increase of MDA, and limiting the decrease of SOD and GSH (Lin et al., 2019). In another study, flavonoids from G. pentaphyllum were extracted and tested on human lung carcinoma A549 cells. It was found that the flavonoids protected A549 cells against hydrogen peroxide-induced oxidative damage by increasing the expression levels members of the endogenous antioxidant system including SOD, GSH, Nrf2, NQO1, and HO-1 (Wang et al., 2018). Another study evaluated the antioxidant potential of one G. pentaphyllum component, the phytoestrogen gypenoside XVII, it was found that the phytoestrogen alleviated atherosclerosis via the ER $\alpha$-mediated PI3K/Akt pathway (Yang et al., 2017). A prior study evaluated the effects of gypenosides of G. pentaphyllum on hydrogen peroxideinduced oxidative damage in bovine pulmonary artery ECs. The gypenosides protected the ECs from oxidative injury further suggesting its potent antioxidant activity as well as its prospective use as a preventative supplement against atherosclerosis ( $\mathrm{Li}$ and Lau, 1993).

Inflammation can contribute to the onset of atherosclerosis and other CVD risk factors, hence, reducing inflammation can act as a protective factor in CVDs. The gypenoside XLIX (Gyp-XLIX) from G. pentaphyllum has been studied for its anti-inflammatory properties. Gyp-XLIX inhibited LPS- and TNF- $\alpha$-induced NF- $\kappa B$ activation in THP-1 monocytes and in HUVECs. Gyp-XLIX inhibition of NF- $\kappa \mathrm{B}$ activation appears to be through a PPAR- $\alpha-$ dependent pathway (Huang et al., 2006). On the other hand, contradictory results were reported by Aktan et al., where $G$. pentaphyllum gypenosides attenuated NF- $\kappa \mathrm{B}$ activation. In fact, the gypenosides extracted from G. pentaphyllum could suppress NO production by inhibiting iNOS activity and levels in murine macrophages. Gypenoside-mediated decrement of iNOS protein expression turned out to be mediated by the inhibition of NF- $\kappa B$ activation (Aktan et al., 2003). In a different study, Tanner et al. showed that G. pentaphyllum could elicit beneficial effects on vascular function by acting as an inducer of eNOS (Tanner et al., 1999).

Attenuating lipid accumulation may decrease CVDs incidence. A study assessed the role of ombuine, a dual agonist of PPAR- $\alpha$ and 
PPAR- $\delta / \beta$ in lipid metabolism. Ombuine or mbuin-3-O- $\beta$ - $d-$ glucopyranoside, a flavonoid from G. pentaphyllum, were applied to HepG2 cells. Ombuine-stimulated HepG2 cells had activated PPAR- $\alpha$ and PPAR- $\delta / \beta$, transcription factors that enhance lipolysis. Ombuine-mediated activation of PPAR- $\alpha$ and PPAR- $\delta / \beta$ significantly reduced intracellular concentrations of triglyceride and cholesterol as well as decreased lipogenic gene expression witnessed as decreased levels of sterol regulatory element binding protein-1c and stearoyl-CoA desaturase-1. These findings further our understanding of how G. pentaphyllum may be involved in lipid metabolism (Malek et al., 2013).

In a study that evaluated the role of total flavonoids of $G$. pentaphyllum on apoptosis in cardiomyocytes of neonatal rats, it was found that hypoxia-reoxygenation (H/R)-cardiomyocytes had an increased protein expression of apoptosis-associated Fas/FasL genes. Flavonoids of $G$. pentaphyllum could protect cardiomyocytes against $\mathrm{H} / \mathrm{R}$ injury by decreasing the production of TNF- $\alpha$ and downregulating the protein levels of Fas/FasL genes leading to inhibition of myocyte apoptosis (Le et al., 2007).

\section{In Vivo Preclinical Evaluations of G. pentaphyllum}

Gypenosides are G. pentaphyllum key components with the ability to help prevent atherosclerosis. The anti-atherosclerotic effects of a mixture (HG) of Fermentum rubrum Hongqu and $G$. pentaphyllum gypenosides were investigated in Wistar rats. The study results revealed that the $H G$ mixture had antiatherosclerotic effects that were better than statin, simvastatin, treatment highlighting the anti-atherosclerotic potential of $G$. pentaphyllum (Gou et al., 2018). In addition, HG alleviated oxidative stress biomarkers by restoring antioxidant defense components and decreasing the serum levels of antiinflammatory cytokines in male Sprague-Dawley rats with fatty liver disease. The HG mixture in this study displayed atheroprotective characteristics (Gou et al., 2016).

Emphasizing the reported G. pentaphyllum gypenoside antiinflammatory and antioxidant capabilities, $\mathrm{Yu}$ et al. demonstrated their beneficial effects in an ischemia-reperfusion injury rat model where they were found to inhibit apoptosis. Ischemia-reperfusion injury has detrimental outcomes in CHD. Yu et al. found that in ischemia reperfusion injured-rats, the administration of gypenosides decreased apoptotic rates as well as improved cardiac function. Gypenosides inhibited ER-stress and apoptosis through the blockade of the CHOP pathway and the activation of PI3K/Akt pathway (Yu et al., 2016b). In a different study, it was demonstrated that pretreatment with gypenosides limited the infarct size and relieved ischemia reperfusion-induced pathological changes in the myocardium, also in an ischemia reperfusion injury rat model. Additionally, left ventricle function was preserved. Molecularly, gypenosides pre-treatment reduced oxidative stress and restored the antioxidant machinery in the myocardium. The cardioprotective effects were also evidenced by the preservation of mitochondrial function in myocytes. In this regard, the maintenance of the mitochondrial membrane integrity inhibited the release of cytochrome $\mathrm{c}$ from the mitochondria into the cytosol. This further demonstrated the role of gypenosides from $G$. pentaphyllum as a cytoprotective agent against acute myocardial infarction and reperfusion injury (Yu et al., 2016a).

As elucidated to earlier, diabetes is positively correlated with the development of CVDs. The effect of G. pentaphyllum extracts on fasting blood sugar levels in diabetic mice was assessed. It was found that the extracts had inhibitory effects on $\alpha$-glucosidase activity while affecting the protein expression of GLUT2 which highlights its potential to manage diabetes (Wang et al., 2019).

Attenuating obesity, a risk factor for developing CVDs, may decrease CVD incidence. G. pentaphyllum is largely used for the management of diseases such as hyperlipidemia, fatty liver, and obesity in China. G. pentaphyllum was found to affect lipid metabolism and elicit anti-hyperlipidemic effects by elevating the levels of phosphatidylcholine and decreasing the levels of trimethylamine $\mathrm{N}$-oxide in the plasma and liver of rats (Wang et al., 2013). In addition, Gauhar et al. studied the effects of heat processed ethanol extracts of G. pentaphyllum on obese mice. They found that this extract decreased obesity in $o b / o b$ mice by activating the AMP-activated protein kinase (AMPK) pathway. This study suggested a possible mechanism for fat-loss as well as a potential for the use of G. pentaphyllum as a weight-loss supplement (Gauhar et al., 2012). Gypenosides antihyperlipidemic effects were examined in rats with poloxamer P407-induced hyperlipidemia. Gypenosides at $250 \mathrm{mg} / \mathrm{kg}$ of body weight was orally administered to hyperlipidemic rats. Four and 12 days of gypenosides administration reduced plasma triglycerides levels by $53 \%$ and $85 \%$, respectively. Similarly, total cholesterol levels were decreased by $10 \%$ and $44 \%$, respectively. Interestingly enough, results were similar to atorvastatin cholesterol-lowering statin drugs. Additionally, LDL levels were reduced and HDL levels were increased by gypenoside, which also reversed the poloxamer $\mathrm{P} 407$ inhibition of lipoprotein lipase activity. This shows a promising therapeutic potential of G. pentaphyllum for lowering high triglyceride and cholesterol levels during acute hyperlipidemia (Megalli et al., 2005). In accordance, extracts from the plant also decreased triglyceride levels and LDL levels in obese Zucher rats (Megalli et al., 2006). By employing a new extraction technique, Lee et al. described some biological activity of a $G$. pentaphyllum extract with a higher content of gypenoside $\mathrm{L}(1.8 \% \mathrm{w} / \mathrm{w})$, gypenoside LI (1.4\% w/w), and ginsenoside Rg3 (0.15\% w/w) (Lee et al., 2019b). While HFD-fed mice showed significant clinical effects such as increases in body weight, fat mass, white adipose tissue, and adipocyte hypertrophy as compared to the control group, the GPE-treated group failed to show them. GPE treatment also reduced serum levels of triglyceride, total cholesterol, and LDLcholesterol, without affecting HDL-cholesterol. Mechanistically, the clinically observed GPE effects appeared due to increased AMPK activation and suppressed adipogenesis by decreasing the mRNA levels of CCAAT/enhancer binding protein- $\alpha(\mathrm{C} / \mathrm{EBP} \alpha)$, PPAR $\gamma$, SREBP-1c, PPAR $\gamma$ coactivator- $1 \alpha$, fatty acid synthase, adipocyte protein 2 , and sirtuin 1 , and increased levels of 
carnitine palmitoyltransferase and hormone-sensitive lipase (Lee et al., 2019b).

\section{G. pentaphyllum to the Clinic}

In general, there are few human trials that addressed $G$. pentaphyllum extract therapeutic effects or safety. A search on clinicaltrials.gov shows that there are only four studies that use G. pentaphyllum extracts. One of the studies is on obese patients and three are on diabetes mellitus patients.

Actiponin, an extract of G. pentaphyllum, is a dietary supplement used for weight loss in obese individuals. During an interventional study, 80 randomized Korean participants took part in a double blind, parallel study for 12 weeks where the experimental group took Actiponin at a dose of $450 \mathrm{mg} /$ day. The experimental group lost weight with no adverse effects, as compared to the placebo group (Park S. et al., 2014).

In another study, $1 \mathrm{mg} / \mathrm{kg}$ of the water extract of $G$. pentaphyllum was given to 44 patients with CVDs and 56 healthy individuals and the platelet aggregation was studied. It was revealed that the water extract elicited significant inhibition of the aggregation of platelets. This means that there is potential for the use of this supplement to prevent cardio-cerebrovascular diseases while being cautious not to give these supplements to patients who suffer from low platelet count or bleeding disorders (Juan and Shanzhang, 1995).

Some studies suggest there is a link between anxiety disorders and an increased risk of developing a CVD (Fan et al., 2008; Vogelzangs et al., 2010; Seldenrijk et al., 2011; Batelaan et al., 2014). For this reason, it is of interest to reduce anxiety in order to decrease the risk of developing CVD in predisposed individuals. It was shown that $G$. pentaphyllum ethanol extract had anti-anxiety effects on mice exposed to chronic stress (Choi H. et al., 2013). This finding was replicated in a double-blind, placebo-controlled clinical trial that had 72 healthy Korean individuals under chronic stressful conditions. Thirty-six participants were given $200 \mathrm{mg}$ of G. pentaphyllum ethanol extract, twice a day for 8 weeks. The supplementation reduced the experimental group anxiety without any adverse drug effects suggesting its potential as a safe antianxiety supplement (Choi et al., 2019).

Although the proven pharmacological effects of $G$. pentaphyllum in in vitro studies and in vivo animal studies may not necessarily translate well into efficacy human subjects, there are positive studies. For instance, a set of studies conducted in T2DM patients supplemented with G. pentaphyllum tea showed improvements in insulin sensitivity and glycemia with no adverse side effects (Huyen et al., 2010; Huyen et al., 2013). In one of the trials, 24 drug-naïve T2DM patients were randomized to take either $6 \mathrm{~g}$ daily of Gynostemma pentaphyllum tea or placebo tea, during 1- week period. The authors measured FPG, insulin levels, and HbAlc levels before, during, and after the treatment. The study showed a prompt improvement of glycemia and insulin sensitivity, and suggested that Gynostemma pentaphyllum tea could be an effective, and safe approach to treat T2DM patients (Huyen et al., 2010). The same authors followed up with another study that used the same study design but enrolled only 16 drug-naïve T2DM patients. The authors measured the same parameters and came to the same conclusion that Gynostemma pentaphyllum tea exerted antidiabetic effects by improving insulin sensitivity (Huyen et al., 2013). These results were confirmed by another study where $G$. pentaphyllum was used together with sulfonylurea (Huyen et al., 2012). Thus far, the current data indicate that $G$. pentaphyllum is quite efficient at improving insulin sensitivity and blood sugar levels if administered solely and that its efficacy may be enhanced when combined with other medications.

\section{Safety, Toxicity, and Side Effects of G. pentaphyllum}

In a study evaluating the toxicity of $G$. pentaphyllum extract on female Sprague-Dawley rats, a single dose of up to $5000 \mathrm{mg} / \mathrm{kg}$ of the extract was given and subchronic toxicity tests were performed with $1000 \mathrm{mg} / \mathrm{kg} /$ day for 90 days. No rat death occurred nor did any signs of toxicity arise. Blood chemistry values, though statistically different from the control group, were within normal ranges in rats. Thus, no mortality nor abnormalities have risen from the G. pentaphyllum extract treatment (Chiranthanut et al., 2013). In addition, no toxicity or mortality was reported upon long-term administration of a dose up to $750 \mathrm{mg} / \mathrm{kg}$ body weight of G. pentaphyllum in rats (Attawish et al., 2004).

A Phase I clinical trial was conducted to evaluate the safety of G. pentaphyllum whereby three groups of healthy volunteers were administered 50, 200, and $400 \mathrm{mg}$ twice daily with water extract of $G$. pentaphyllum for two months. No major immune adverse events such as significant changes in natural killer cell activities, number of $\mathrm{CD} 3+, \mathrm{CD} 4+$, and CD8+ cells, were reported. No biochemical parameters were significantly affected either. Such doses of G. pentaphyllum were deemed to be safe (Chavalittumrong et al., 2007). In another clinical trial, 537 bronchitic patients were treated three times a day with $G$. pentaphyllum (2.5-3 g, prepared as tablets or capsules). Adverse side effects that included vomiting, abdomen tension, diarrhea (or constipation), dizziness, blurred vision, and tinnitus effects were seen in a small number of patients. Notably, these symptoms were mild and did not stop the patients from taking the G. pentaphyllum extract (Razmovski-Naumovski et al., 2005). A very recent randomized, double-blind, placebo-controlled clinical trial utilizing G. pentaphyllum extract in 72 healthy adults revealed no adverse side effects of the ingestion of the ethanolic extract of G. pentaphyllum (Choi et al., 2019). Overall, consumption of $G$. pentaphyllum seems to be safe at the doses required to observe a therapeutic effect.

\section{CONCLUSION}

Despite the abundance of knowledge regarding CVDs, CVD prevalence continues to be on the rise. Thus, there is an immediate demand for new safe, effective, and relatively cheap drug candidates. Mounting evidence obtained from in vitro and in vivo studies suggests that the four traditionally used medicinal 
A

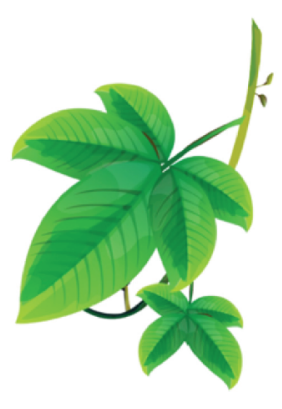

B<smiles>CC(C)=CCC[C@](C)(O)C1CC[C@@]2(C)C1C(O)CC1[C@@]3(C)CC[C@H](O)C(C)(C)C3CC[C@]12C</smiles>

\begin{tabular}{|c|c|c|}
\hline Gypenoside & $\mathbf{R}_{1}$ & $\mathbf{R}_{2}$ \\
\hline 201 S-Protopanaxadiol (PPD) & $\mathrm{H}$ & $\mathrm{H}$ \\
\hline Gypenoside III & $\beta$-D-Glu $(1 \rightarrow 2) \beta$-D-Glu & 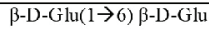 \\
\hline Gypenoside VIII & $\beta$-D-Glu $(1 \rightarrow 2) \beta$-D-Glu & $\beta$-D-Glu \\
\hline
\end{tabular}

FIGURE 5 | Gynostemma pentaphyllum. (A) Gynostemma pentaphyllum (Source https://pngtree.com/freepng). Examples of the chemical structure of Gynostemma pentaphyllum Gypenoside that are usually synthesized 20 S-Protopanaxadiol (PPD). (B) example of some chemical structures of Gypenosides.

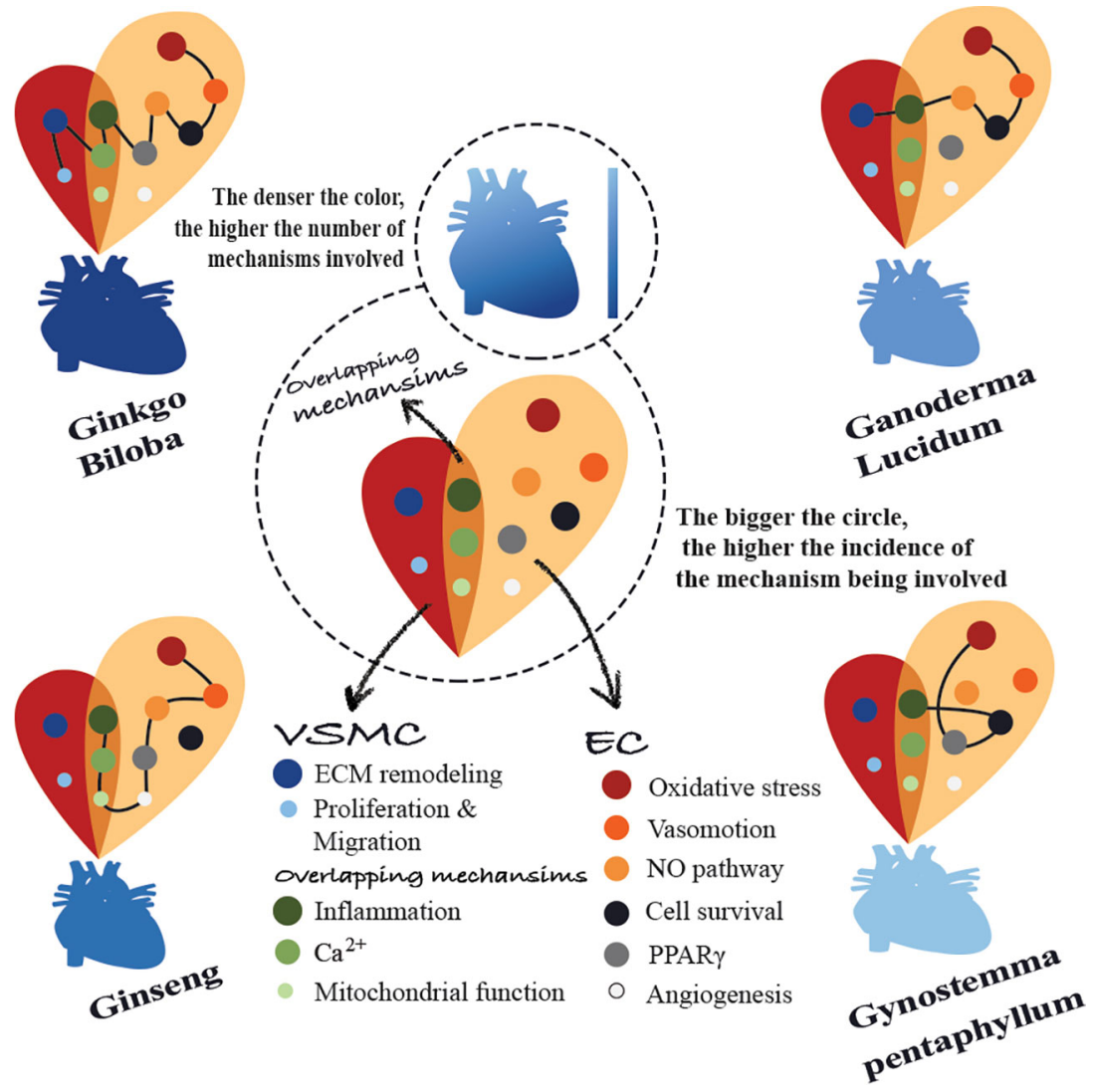

FIGURE 6 | Herbal therapies in the context of CVDs. Herbal preparations can exert protective effects by ameliorating the pathological effects exerted by CVDs risk factors. The herbal extracts can attenuate endothelial dysfunction and/or VSMC alterations by acting as, vasodilators, ROS scavengers, anti-oxidants, antiinflammatory, anti-apoptotic, anti-hypertrophic, or anti-proliferative agents. This achieved through mechanisms that act in ECs only, VSMCs only, or through overlapping mechanism that act in both ECs and VSMCs. In ECs, herbal preparations can increase NO availability, decrease mitochondrial dysfunction and/or metabolic abnormalities as well as enhance angiogenesis. This can decrease the incidence of atherosclerosis and hypertension, which in return can decrease the risk of CVDs development. In VSMCs, the herbal extracts can modulate ECM deposition as well as cell migration, proliferation, and cell shape changes. VSMC, vascular smooth muscle cell; ECM, extracellular matrix; EC, endothelial cell; NO, nitric oxide; PPARY, peroxisome proliferator-activated receptor-gamma. 
TABLE 2 | Summary of the mechanisms of action of the four discussed plants.

\begin{tabular}{|c|c|c|c|}
\hline Plant & & Mechanism of action & References \\
\hline Ginseng & $\begin{array}{l}\text { Bioactive/active fractions: } \\
\mathrm{Rb} 1, \mathrm{Rg} 1, \mathrm{Rg} 3, \mathrm{Re}, \text { and } \mathrm{Rd} \\
\text { Commonly used extracts: } \\
\text { Panax ginseng, Panax notoginseng, Panax } \\
\text { quinquefolium L., Panax japonicas }\end{array}$ & 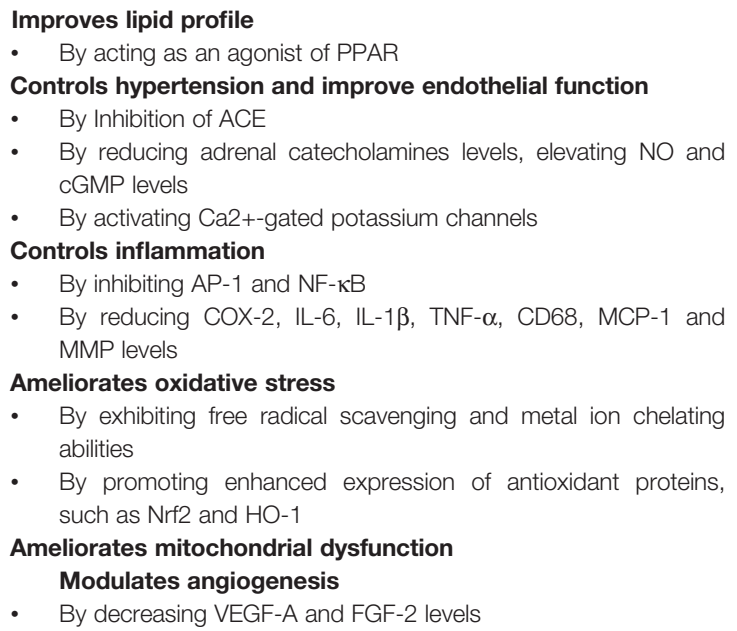 & $\begin{array}{l}\text { (Kim et al., 1999b; Keum et al., } \\
\text { 2003; Park et al., 2005; Persson } \\
\text { et al., 2006; Shin Y. et al., 2013; } \\
\text { Park J. et al., 2014; Lee H. et al., } \\
\text { 2016; Deng et al., 2017; Singh } \\
\text { et al., 2017; Lee et al., 2019a) }\end{array}$ \\
\hline Gingko Biloba & $\begin{array}{l}\text { Bioactive/active fractions: } \\
\text { Ginkgolides classified into either A, B, C, J, } \\
\text { or M types } \\
\text { Commonly used extracts: } \\
\text { EGb761 }\end{array}$ & $\begin{array}{l}\text { Improves lipid profile } \\
\text { - } \quad \text { By decreasing PPARs levels } \\
\text { Controls hypertension and improves endothelial function } \\
\text { - } \quad \text { By decreasing ACE activity, activating cholinergic pathways, } \\
\text { limiting LPS-induced proliferation of VSMCs } \\
\text { - By decreasing ICAM-1 and VCAM-1 expression, decreasing } \\
\text { phosphorylation of Akt/FoxO3a } \\
\text { - By restoring eNOS activity, decreasing iNOS expression and } \\
\text { consequently elevating NO levels } \\
\text { Controls inflammation } \\
\text { - By suppressing TLR-4 expression } \\
\text { - By decreasing MMP-1, MCP-1, TNF- } \alpha \text {, IL-6, or IL-1 } \beta \\
\text { Ameliorates oxidative stress } \\
\text { - By decreasing NOX activity and level, activating endogenous } \\
\text { - } \text { Akt/Nrf2 antioxidant stress pathway } \\
\text { - By increasing levels of HO-1, SOD and GSH-Px } \\
\text { Prevents hypertrophy } \\
\text { - By activating M2 muscarinic receptors/NO pathway } \\
\text { - By decreasing calcium overload and inhibiting the } \mathrm{Na}^{+} / \mathrm{Ca}^{2+} \\
\text { Prevents apoptosis } \\
\text { - By decreasing caspase } 3 \text { and pro-apoptotic Bax expression } \\
\text { and increasing anti-apoptotic Bcl-2 expression }\end{array}$ & $\begin{array}{l}\text { (Akiba et al., 2007; Lin et al., } \\
\text { 2007; Mansour et al., 2011; Liu } \\
\text { et al., 2013; Liou et al., 2015; } \\
\text { Mesquita et al., 2017; Abdel- } \\
\text { Zaher et al., 2018; Huang et al., } \\
\text { 2018; Chen et al., 2019). }\end{array}$ \\
\hline $\begin{array}{l}\text { Ganoderma } \\
\text { lucidum }\end{array}$ & $\begin{array}{l}\text { Bioactive/active fractions: } \\
\text { ganoderic acids } A, B, C, D, F, H, K, S \text {, and } \\
\text { Y, } \beta \text {-d-Glucan polysaccharides } \\
\text { Commonly used extracts: } \\
\text { Polysaccharide peptide (PsP) Ganoderma } \\
\text { lucidum }\end{array}$ & $\begin{array}{l}\text { Controls hypertension and improves endothelial function } \\
\text { - By inhibition of ACE, enhancing Angiotensin 1-mediated } \\
\text { phosphorylation of eNOS } \\
\text { - } \text { By reducing the levels of vasoconstrictor peptide Endothelin-1 } \\
\text { Improves lipid profile } \\
\text { - By upregulating lipid metabolism (ACOX1 and ACC) } \\
\text { Ameliorates oxidative stress and inflammation } \\
\text { - By enhancing phosphorylation of Nrf2 which upregulates HO-1, } \\
\text { GST, NQO-1, SOD, CAT, GSH-Px and GSH } \\
\text { By decreasing the levels of MDA and ICAM, and regulation of } \\
\text { mTOR/S6K signaling pathways } \\
\text { Reduces necrosis } \\
\text { - By decreasing the levels of creatine phosphokinase }\end{array}$ & $\begin{array}{l}\text { (Hikino et al., 1985; Tomoda } \\
\text { et al., 1986; Shi et al., 2002; Shi } \\
\text { et al., 2010; Lasukova et al., } \\
\text { 2015; Wang et al., 2015; } \\
\text { Wihastuti and Heriansyah, 2017; } \\
\text { Cuong et al., 2019; Wu et al., } \\
\text { 2019) }\end{array}$ \\
\hline
\end{tabular}


TABLE 2 | Continued

\begin{tabular}{|c|c|c|c|}
\hline Plant & & Mechanism of action & References \\
\hline $\begin{array}{l}\text { Gynostemma } \\
\text { pentaphyllum }\end{array}$ & $\begin{array}{l}\text { Bioactive/active fractions: } \\
\text { dammarane-type triterpene saponins } \\
\text { (gypenosides or gynosaponins) } \\
\text { Commonly used extracts: } \\
\text { Actiponin, Ombuine }\end{array}$ & 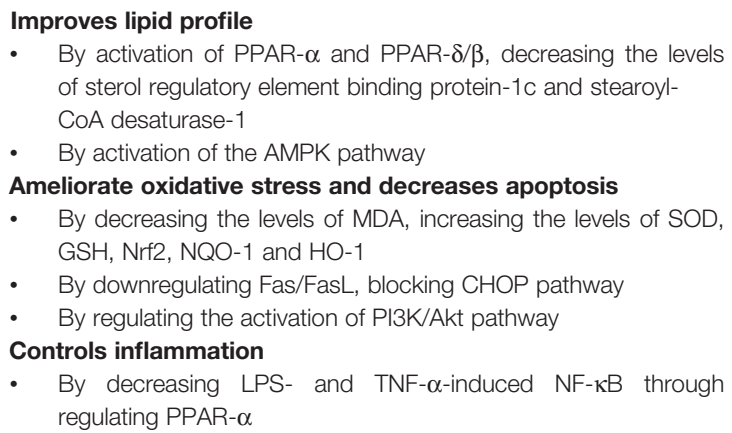 & $\begin{array}{l}\text { (Huang et al., 2006; Megalli } \\
\text { et al., 2006; Malek et al., 2013; } \\
\text { Yu et al., 2016b; Yang et al., } \\
\text { 2017; Wang et al., 2018; Lin } \\
\text { et al., 2019) }\end{array}$ \\
\hline
\end{tabular}

PPAR, peroxisome proliferator-activated receptor; ACE, acetylcholinesterase; NO, nitric oxide; AP-1, activator protein 1; NF- $\kappa B$, nuclear factor kappa-light-chain-enhancer of activated B cells; COX, cyclooxygenase; IL, interleukin; TNF, tumor necrosis factor; CD, cluster of differentiation; MCP, monocyte chemoattractant; MMP, matrix metalloproteases; Nrf2, nuclear factor erythroid-2-related factor 2; HO-1, hemeoxygenase-1; VEGF, vascular endothelial growth factor; FGF, fibroblast growth factor; VSMCs, vascular smooth muscle cells; ICAM, intercellular adhesion molecule; VCAM, vascular cell adhesion molecule; Akt, protein kinase B; FoxO3a, forkhead box O; eNOS, endothelial nitric oxide synthase; iNOS, inducible nitric oxide synthase; TLR, toll-like receptor; NOC, nicotinamide adenine dinucleotide phosphate oxidase; SOD, superoxide dismutase; GSH-Px, glutathione peroxidase; MAPK, mitogen-activated protein kinase; ACOX1, peroxisomal acyl-coenzyme A oxidase 1; ACC, acetyl-CoA carboxylase; GST, glutathione S-transferase; NQO-1, NAD(P)H dehydrogenase (quinone); GSH, glutathione; MDA, malondialdehyde; mTOR, mammalian target of rapamycin complex; S6K, S6 kinase; AMPK, AMP-activated protein kinase; CHOP, C/EBP homologous protein.

plants discussed in this review significantly modulate key cellular, molecular, and metabolic mechanisms that control both CVDs pathogenesis and pathophysiology (Figure 6). Here are presented the recent findings, advances, and studies describing the therapeutic values of these plants in the context of several CVDs. Current evidence demonstrates that these herbal medicines have potent therapeutic properties and can ameliorate pathological conditions associated with CVDs (Table 2 and Figure 6). However, clear clinical therapeutic benefits have not yet been secured. As such, these herbal treatments cannot be safely recommended as an alternative therapeutic medicine. In fact, the safety and toxicity of some of these plants have recently raised potential concerns (eg. Gingko Biloba). We conclude that better-designed studies and future clinical trials involving larger sample sizes are needed to investigate the role of different medicinal plants and their underlying mechanisms in the

\section{REFERENCES}

Abdelazim, A., Khater, S., Ali, H., Shalaby, S., Afifi, M., Saddick, S., et al. (2018). Panax ginseng improves glucose metabolism in streptozotocin-induced diabetic rats through 5'Adenosine mono phosphate kinase up-regulation. Saudi J. Biol. Sci. 26 (7), 1436-1441. doi: 10.1016/j.sjbs.2018.06.001

Abdel-Zaher, A. O., Farghaly, H. S., El-Refaiy, A. E., and Abd-Eldayem, A. M. (2018). Protective effect of the standardized leaf extract of $G$ inkgo biloba (EGb761) against hypertension-induced renal injury in rats. Clin. Exp. Hypertens. 40 (8), 1-12. doi: 10.1016/j.sjbs.2018.06.001

Abegaz, T. M., Tefera, Y. G., and Befekadu Abebe, T. (2017). Target Organ Damage and the Long Term Effect of Nonadherence to Clinical Practice Guidelines in Patients with Hypertension: A Retrospective Cohort Study. Int. J. Hypertens. 2017, 2637051. doi: 10.1155/2017/2637051

Aday, A. W., and Ridker, P. M. (2018). Antiinflammatory Therapy in Clinical Care: The CANTOS Trial and Beyond. Front. Cardiovasc. Med. 5, 62. doi: $10.3389 /$ fcvm.2018.00062

Ahmad, M. F. (2018). Ganoderma lucidum: Persuasive biologically active constituents and their health endorsement. BioMed. Pharmacother. 107, 507-519. doi: 10.1016/j.biopha.2018.08.036 context of CVDs. Above all, the future clinical trials should address the safety and toxicity of these herbal remedies.

\section{AUTHOR CONTRIBUTIONS}

AS ideated and wrote the manuscript. DT, HP, TN, and GN contributed to manuscript writing and editing. $\mathrm{SAH}, \mathrm{HH}$, and $\mathrm{SH}$ contributed to manuscript writing and figures drawing. $\mathrm{AE}$ and GP contributed to manuscript ideation, revision, and editing

\section{FUNDING}

This work has been made possible thanks to grants (Ager S.O.S.) and (fondo di Ateneo per la ricerca 2019) to GP and Qatar University grant (IRCC-2019-007) to GN and GP.

Akiba, S., Yamaguchi, H., Kumazawa, S., Oka, M., and Sato, T. (2007). Suppression of oxidized LDL-induced PDGF receptor beta activation by ginkgo biloba extract reduces MMP-1 production in coronary smooth muscle cells. J. Atheroscler. Thromb. 14 (5), 219-225. doi: 10.5551/jat.e516

Aktan, F., Henness, S., Roufogalis, B. D., and Ammit, A. J. (2003). Gypenosides derived from Gynostemma pentaphyllum suppress NO synthesis in murine macrophages by inhibiting iNOS enzymatic activity and attenuating NF-kappaB-mediated iNOS protein expression. Nitric Oxide 8 (4), 235-242. doi: 10.1016/S1089-8603(03)00032-6

Al Disi, S. S., Anwar, M. A., and Eid, A. H. (2016). Anti-hypertensive herbs and their mechanisms of action: part I. Front. Pharmacol. 6, 323. doi: 10.3389/ fphar.2015.00323

Al-Shehabi, T. S., Iratni, R., and Eid, A. H. (2016). Anti-atherosclerotic plants which modulate the phenotype of vascular smooth muscle cells. Phytomedicine 23 (11), 1068-1081. doi: 10.1016/j.phymed.2015.10.016

Anwar, M. A., Al Disi, S. S., and Eid, A. H. (2016). Anti-Hypertensive Herbs and Their Mechanisms of Action: Part II. Front. Pharmacol. 7, 50. doi: 10.3389/fphar.2016.00050

Arenz, A., Klein, M., Fiehe, K., Gross, J., Drewke, C., Hemscheidt, T., et al. (1996). Occurrence of neurotoxic 4'-O-methylpyridoxine in Ginkgo biloba leaves, Ginkgo medications and Japanese Ginkgo food. Planta Med. 62 (6), 548551. doi: 10.1055/s-2006-957967 
Ashraf, R., Khan, R. A., Ashraf, I., and Qureshi, A. A. (2013). Effects of Allium sativum (garlic) on systolic and diastolic blood pressure in patients with essential hypertension. Pak. J. Pharm. Sci. 26 (5), 859-863.

Attawish, A., Chivapat, S., Phadungpat, S., Bansiddhi, J., Techadamrongsin, Y., Mitrijit, O., et al. (2004). Chronic toxicity of Gynostemma pentaphyllum. Fitoterapia 75 (6), 539-551. doi: 10.1016/j.fitote.2004.04.010

August, P. (2004). Overview: mechanisms of hypertension: cells, hormones, and the kidney. J. Am. Soc. Nephrol. 15 (8), 1971-1973. doi: 10.1097/01.Asn.0000133197.23478.76

Aziz, T. A., Hussain, S. A., Mahwi, T. O., Ahmed, Z. A., Rahman, H. S., and Rasedee, A. (2018). The efficacy and safety of Ginkgo biloba extract as an adjuvant in type 2 diabetes mellitus patients ineffectively managed with metformin: a double-blind, randomized, placebo-controlled trial. Drug Des. Devel. Ther. 12, 735-742. doi: 10.2147/dddt.S157113

Baek, K. S., Yi, Y. S., Son, Y. J., Yoo, S., Sung, N. Y., Kim, Y., et al. (2016). In vitro and in vivo anti-inflammatory activities of Korean Red Ginseng-derived components. J. Ginseng Res. 40 (4), 437-444. doi: 10.1016/j.jgr.2016.08.003

Bang, H., Kwak, J. H., Ahn, H. Y., Shin, D. Y., and Lee, J. H. (2014). Korean red ginseng improves glucose control in subjects with impaired fasting glucose, impaired glucose tolerance, or newly diagnosed type 2 diabetes mellitus. J. Med. Food 17 (1), 128-134. doi: 10.1089/jmf.2013.2889

Batelaan, N. M., ten Have, M., van Balkom, A. J., Tuithof, M., and de Graaf, R. (2014). Anxiety disorders and onset of cardiovascular disease: the differential impact of panic, phobias and worry. J. Anxiety Disord. 28 (2), 252-258. doi: 10.1016/j.janxdis.2013.12.003

Belwal, T., Giri, L., Bahukhandi, A., Tariq, M., Kewlani, P., Bhatt, I. D., et al. (2019). "Ginkgo biloba," in Nonvitamin and Nonmineral Nutritional Supplements, vol. 241-250. (San Diego, California, United States: Elsevier).

Benjamin, E. J., Muntner, P., Alonso, A., Bittencourt, M. S., Callaway, C. W., Carson, A. P., et al. (2019). Heart Disease and Stroke Statistics-2019 Update: A Report From the American Heart Association. Circulation 139 (10), e56-e528. doi: 10.1161/cir.0000000000000659

Bent, S., Goldberg, H., Padula, A., and Avins, A. L. (2005). Spontaneous bleeding associated with ginkgo biloba: a case report and systematic review of the literature: a case report and systematic review of the literature. J. Gen. Internal Med. 20 (7), 657-661. doi: 10.1111/j.1525-1497.2005.0121.x

Bidani, A. K., and Griffin, K. A. (2004). Pathophysiology of hypertensive renal damage: implications for therapy. Hypertension 44 (5), 595-601. doi: 10.1161/ 01.Hyp.0000145180.38707.84

Boh, B., Berovic, M., Zhang, J., and Zhi-Bin, L. (2007). Ganoderma lucidum and its pharmaceutically active compounds. Biotechnol. Annu. Rev. 13, 265-301. doi: 10.1016/S1387-2656(07)13010-6

Bonassi, S., Prinzi, G., Lamonaca, P., Russo, P., Paximadas, I., Rasoni, G., et al. (2018). Clinical and genomic safety of treatment with Ginkgo biloba L. leaf extract (IDN 5933/Ginkgoselect(R)Plus) in elderly: a randomised placebocontrolled clinical trial [GiBiEx]. BMC Complement. Alternat. Med. 18 (1), 22. doi: 10.1186/s12906-018-2080-5

Bridi, R., Crossetti, F. P., Steffen, V. M., and Henriques, A. T. (2001). The antioxidant activity of standardized extract of Ginkgo biloba (EGb 761) in rats. Phytother. Res. 15 (5), 449-451. doi: 10.1002/ptr.814

Brinkley, T. E., Lovato, J. F., Arnold, A. M., Furberg, C. D., Kuller, L. H., Burke, G. L., et al. (2010). Effect of Ginkgo biloba on blood pressure and incidence of hypertension in elderly men and women. Am. J. Hypertens. 23 (5), 528-533. doi: 10.1038/ajh.2010.14

Brixius, K., Willms, S., Napp, A., Tossios, P., Ladage, D., Bloch, W., et al. (2006). Crataegus special extract WS 1442 induces an endothelium-dependent, NOmediated vasorelaxation via eNOS-phosphorylation at serine 1177. Cardiovasc. Drugs Ther. 20 (3), 177-184. doi: 10.1007/s10557-006-8723-7

Cao, F., Cheng, H., Cheng, S., Li, L., Xu, F., Yu, W., et al. (2012). Expression of selected Ginkgo biloba heat shock protein genes after cold treatment could be induced by other abiotic stress. Int. J. Mol. Sci. 13 (5), 5768-5788. doi: 10.3390/ijms13055768

Caron, M. F., Hotsko, A. L., Robertson, S., Mandybur, L., Kluger, J., and White, C. M. (2002). Electrocardiographic and hemodynamic effects of Panax ginseng. Ann. Pharmacother. 36 (5), 758-763. doi: 10.1345/aph.1A411

Celermajer, D. S. (1997). Endothelial dysfunction: does it matter? Is it reversible? J. Am. Coll. Cardiol. 30 (2), 325-333. doi: 10.1016/s0735-1097(97)00189-7

Chan, K., Chui, S., Wong, D., Ha, W., Chan, C., and Wong, R. (2004). Protective effects of Danshensu from the aqueous extract of Salvia miltiorrhiza (Danshen) against homocysteine-induced endothelial dysfunction. Life Sci. 75 (26), $3157-$ 3171. doi: 10.1016/j.lfs.2004.06.010

Chan, P. C., Xia, Q., and Fu, P. P. (2007). Ginkgo biloba leave extract: biological, medicinal, and toxicological effects. J. Environ. Sci. Health C Environ. Carcinog. Ecotoxicol. Rev. 25 (3), 211-244. doi: 10.1080/10590500701569414

Chang, C. J., Lin, C. S., Lu, C. C., Martel, J., Ko, Y. F., Ojcius, D. M., et al. (2015). Ganoderma lucidum reduces obesity in mice by modulating the composition of the gut microbiota. Nat. Commun. 6, 7489. doi: 10.1038/ncomms8489

Chavalittumrong, P. S. B., Kijphati, R., Jitjuk, B., Treesangsri, W., Phadungpat, S., Boonruad, T., et al. (2007). A phase I trial of Gynostemma pentaphyllum in healthy volunteers. Songklanakarin J. Sci. Technol. 29, 83-93.

Chen, J., and Gilbert, M. (2006). Flora of China (St Louis, MO and Beijing, China: Missouri Botanical Garden Press \& Science Press, Beijing).

Chen, W. Q., Luo, S. H., Ll, H. Z., and Yang, H. (2005). [Effects of ganoderma lucidum polysaccharides on serum lipids and lipoperoxidation in experimental hyperlipidemic rats]. Zhongguo Zhong Yao Za Zhi 30 (17), 1358-1360.

Chen, X. J., Ren, S. M., Dong, J. Z., Qiu, C. G., Chen, Y. W., and Tao, H. L. (2019). Ginkgo biloba extract-761 protects myocardium by regulating Akt/Nrf2 signal pathway. Drug Des. Dev. Ther. 13, 647-655. doi: 10.2147/DDDT.S191537

Chen, S. (1995). A classificatory system and geographical distribution of the genus Gynostemma BL. (Cucurbitaceae). Acta Phytotaxon. Sin. 33, 403- 410.

Cheng, D., Liang, B., and Li, Y. (2013). Antihyperglycemic effect of Ginkgo biloba extract in streptozotocin-induced diabetes in rats. Biomed. Res. Int. 2013, 162724. doi: $10.1155 / 2013 / 162724$

Chiranthanut, N., Teekachunhatean, S., Panthong, A., Khonsung, P., Kanjanapothi, D., and Lertprasertsuk, N. (2013). Toxicity evaluation of standardized extract of Gynostemma pentaphyllum Makino. J. Ethnopharmacol. 149 (1), 228-234. doi: 10.1016/j.jep.2013.06.027

Chiu, A. E., Lane, A. T., and Kimball, A. B. (2002). Diffuse morbilliform eruption after consumption of ginkgo biloba supplement. J. Am. Acad. Dermatol. 46 (1), 145-146. doi: 10.1067/mjd.2001.118545

Choi, H., Zhao, T., Shin, K., Kim, S., Hwang, B., Lee, C., et al. (2013). Anxiolytic effects of herbal ethanol extract from Gynostemma pentaphyllum in mice after exposure to chronic stress. Molecules 18 (4), 4342-4356. doi: 10.3390/molecules 18044342

Choi, J., Kim, T. H., Choi, T. Y., and Lee, M. S. (2013). Ginseng for health care: a systematic review of randomized controlled trials in Korean literature. PloS One 8 (4), e59978. doi: 10.1371/journal.pone.0059978

Choi, E. K., Won, Y. H., Kim, S. Y., Noh, S. O., Park, S. H., Jung, S. J., et al. (2019). Supplementation with extract of Gynostemma pentaphyllum leaves reduces anxiety in healthy subjects with chronic psychological stress: A randomized, double-blind, placebo-controlled clinical trial. Phytomedicine 52, 198-205. doi: 10.1016/j.phymed.2018.05.002

Chou, T. C., Yen, M. H., Li, C. Y., and Ding, Y. A. (1998). Alterations of nitric oxide synthase expression with aging and hypertension in rats. Hypertension 31 (2), 643-648. doi: 10.1161/01.hyp.31.2.643

Chu, T. T., Benzie, I. F., Lam, C. W., Fok, B. S., Lee, K. K., and Tomlinson, B. (2012). Study of potential cardioprotective effects of Ganoderma lucidum (Lingzhi): results of a controlled human intervention trial. Br. J. Nutr. 107 (7), 1017-1027. doi: 10.1017/s0007114511003795

Chua, Y. T., Ang, X. L., Zhong, X. M., and Khoo, K. S. (2015). Interaction between warfarin and Chinese herbal medicines. Singapore Med. J. 56 (1), 11-18. doi: 10.11622/smedj.2015004

Cianfrocca, C., Pelliccia, F., Auriti, A., and Santini, M. (2002). Ginkgo bilobainduced frequent ventricular arrhythmia. Ital. Heart J. 3 (11), 689-691.

Cong, W.-n., Tao, R.-y., Tian, J.-y., Zhao, J., Liu, Q., and Ye, F. (2011). EGb761, an extract of Ginkgo biloba leaves, reduces insulin resistance in a high-fat-fed mouse model. Acta Pharm. Sin. B 1 (1), 14-20. doi: 10.1016/j.apsb.2011.04.006

Coon, J. T., and Ernst, E. (2002). Panax ginseng: a systematic review of adverse effects and drug interactions. Drug Saf. 25 (5), 323-344. doi: 10.2165/ 00002018-200225050-00003

Cooper, R. S., Kaufman, J. S., and Bovet, P. (2017). Global Burden of Disease Attributable to Hypertension. JAMA 317 (19), 2017-2018. doi: 10.1001/ jama.2017.4213

Couto, G. K., Britto, L. R., Mill, J. G., and Rossoni, L. V. (2015). Enhanced nitric oxide bioavailability in coronary arteries prevents the onset of heart failure in rats with myocardial infarction. J. Mol. Cell Cardiol. 86, 110-120. doi: 10.1016/ j.yjmcc.2015.07.017 
Cragg, G. M., and Newman, D. J. (2013). Natural products: a continuing source of novel drug leads. Biochim. Biophys. Acta 1830 (6), 3670-3695. doi: 10.1016/ j.bbagen.2013.02.008

Cuong, V. T., Chen, W., Shi, J., Zhang, M., Yang, H., Wang, N., et al. (2019). The anti-oxidation and anti-aging effects of Ganoderma lucidum in Caenorhabditis elegans. Exp. Gerontol. 117, 99-105. doi: 10.1016/j.exger.2018.11.016

Davies, M. J., Gordon, J. L., Gearing, A. J., Pigott, R., Woolf, N., Katz, D., et al. (1993). The expression of the adhesion molecules ICAM-1, VCAM-1, PECAM, and E-selectin in human atherosclerosis. J. Pathol. 171 (3), 223-229. doi: $10.1002 /$ path. 1711710311

Davison, E. K., and Brimble, M. A. (2019). Natural product derived privileged scaffolds in drug discovery. Curr. Opin. Chem. Biol. 52, 1-8. doi: 10.1016/ j.cbpa.2018.12.007

DeKosky, S. T., Williamson, J. D., Fitzpatrick, A. L., Kronmal, R. A., Ives, D. G., Saxton, J. A., et al. (2008). Ginkgo biloba for prevention of dementia: a randomized controlled trial. JAMA 300 (19), 2253-2262. doi: 10.1001/jama.2008.683

Deng, Z., Wang, Y., Jiang, K., Liu, X., Wu, W., Gao, S., et al. (2006). Molecular cloning and characterization of a novel dehydrin gene from Ginkgo biloba. Biosci. Rep. 26 (3), 203-215. doi: 10.1007/s10540-006-9016-x

Deng, J., Liu, Y., Duan, Z., Zhu, C., Hui, J., Mi, Y., et al. (2017). Protopanaxadiol and protopanaxatriol-type saponins ameliorate glucose and lipid metabolism in type 2 diabetes mellitus in high-fat diet/streptozocin-induced mice. Front. Pharmacol. 8, 506. doi: 10.3389/fphar.2017.00506

Diamond, B. J., and Bailey, M. R. (2013). Ginkgo biloba: indications, mechanisms, and safety. Psychiatr. Clin. North Am. 36 (1), 73-83. doi: 10.1016/ j.psc.2012.12.006

DiBona, G. F. (2013). Sympathetic nervous system and hypertension. Hypertension 61 (3), 556-560. doi: 10.1161/hypertensionaha.111.00633

Dodge, H. H., Zitzelberger, T., Oken, B. S., Howieson, D., and Kaye, J. (2008). A randomized placebo-controlled trial of Ginkgo biloba for the prevention of cognitive decline. Neurology 70 (19 Pt 2), 1809-1817. doi: 10.1212/ 01.wnl.0000303814.13509.db

Dokken, B. B. (2008). The Pathophysiology of Cardiovascular Disease and Diabetes: Beyond Blood Pressure and Lipids. Diabetes Spectr. 21 (3), 160165. doi: $10.2337 /$ diaspect.21.3.160

Dong, H., Ma, J., Li, T., Xiao, Y., Zheng, N., Liu, J., et al. (2017). Global deregulation of ginseng products may be a safety hazard to warfarin takers: solid evidence of ginseng-warfarin interaction. Sci. Rep. 7 (1), 5813. doi: 10.1038/s41598-017-05825-9

Douglas, G., and Channon, K. M. (2014). The pathogenesis of atherosclerosis. Medicine 42 (9), 480-484. doi: 10.1016/j.mpmed.2014.06.011

Dugoua, J. J., Mills, E., Perri, D., and Koren, G. (2006). Safety and efficacy of ginkgo (Ginkgo biloba) during pregnancy and lactation. Can. J. Clin. Pharmacol. 13 (3), e277-e284.

Egan, B. M., Kjeldsen, S. E., Grassi, G., Esler, M., and Mancia, G. (2019). The global burden of hypertension exceeds 1.4 billion people: should a systolic blood pressure target below 130 become the universal standard? J. Hypertens. 37 (6), 1148-1153. doi: 10.1097/HJH.0000000000002021

Eisenberg, D. M., Davis, R. B., Ettner, S. L., Appel, S., Wilkey, S., Van Rompay, M., et al. (1998). Trends in alternative medicine use in the United States 1990-1997: results of a follow-up national survey. JAMA 280 (18), 1569-1575. doi: 10.1001/jama.280.18.1569

Fabricant, D. S., and Farnsworth, N. R. (2001). The value of plants used in traditional medicine for drug discovery. Environ. Health Perspect. 109 (Suppl 1), 69-75. doi: 10.1289/ehp.01109s169

Fan, A. Z., Strine, T. W., Jiles, R., and Mokdad, A. H. (2008). Depression and anxiety associated with cardiovascular disease among persons aged 45 years and older in 38 states of the United States 2006. Prev. Med. 46 (5), 445-450. doi: 10.1016/j.ypmed.2008.02.016

Fan, X., Zhu, M., Chi, C., Yu, S., Xiong, J., Lu, Y., et al. (2017). Association of arteriosclerosis and/or atherosclerosis with hypertensive target organ damage in the community-dwelling elderly Chinese: the Northern Shanghai Study. Clin. Interv. Aging 12, 929-936. doi: 10.2147/cia.S133691

Farnsworth, N. R., Akerele, O., Bingel, A. S., Soejarto, D. D., and Guo, Z. (1985). Medicinal plants in therapy. Bull. World Health Organ. 63 (6), 965-981.
Feng, Z., Yang, X., Zhang, L., Ansari, I. A., Khan, M. S., Han, S., et al. (2018). Ginkgolide B ameliorates oxidized low-density lipoprotein-induced endothelial dysfunction via modulating Lectin-like ox-LDL-receptor-1 and NADPH oxidase 4 expression and inflammatory cascades. Phytother. Res. 32 (12), 2417-2427. doi: 10.1002/ptr.6177

Fleming, R. M. (2002). The effect of high-, moderate-, and low-fat diets on weight loss and cardiovascular disease risk factors. Prev. Cardiol. 5 (3), 110-118. doi: 10.1111/j.1520-037X.2002.01231.x

Forstermann, U., and Munzel, T. (2006). Endothelial nitric oxide synthase in vascular disease: from marvel to menace. Circulation 113 (13), 1708-1714. doi: 10.1161/CIRCULATIONAHA.105.602532

Fox, C. S., Pencina, M. J., Wilson, P. W., Paynter, N. P., Vasan, R. S., and D'Agostino, R. B. (2008). Lifetime risk of cardiovascular disease among individuals with and without diabetes stratified by obesity status in the Framingham heart study. Diabetes Care 31 (8), 1582-1584. doi: 10.2337/ dc08-0025

Franceschi, C., Bonafe, M., Valensin, S., Olivieri, F., De Luca, M., Ottaviani, E., et al. (2000). Inflamm-aging. An evolutionary perspective on immunosenescence. Ann. N. Y. Acad. Sci. 908, 244-254. doi: 10.1111/j.1749-6632.2000.tb06651.x

Frishman, W. H., Beravol, P., and Carosella, C. (2009). Alternative and complementary medicine for preventing and treating cardiovascular disease. Dis. Mon. 55 (3), 121-192. doi: 10.1016/j.disamonth.2008.12.002

Fu, Y., and Ji, L. L. (2003). Chronic ginseng consumption attenuates age-associated oxidative stress in rats. J. Nutr. 133 (11), 3603-3609. doi: 10.1093/jn/133.11.3603

Fulton, D. J., and Barman, S. A. (2016). Clarity on the Isoform-Specific Roles of NADPH Oxidases and NADPH Oxidase-4 in Atherosclerosis. Arterioscler. Thromb. Vasc. Biol. 36 (4), 579-581. doi: 10.1161/ATVBAHA.116.307096

Gao, Y., Chen, G., Dai, X., Ye, J., and Zhou, S. (2004). A phase I/II study of ling zhi mushroom Ganoderma lucidum (W. Curt.: Fr.) Lloyd (Aphyllophoromycetideae) extract in patients with coronary heart disease. Int. J. Med. Mushrooms 6 (4).

Gao, S., Liu, Z., Li, H., Little, P. J., Liu, P., and Xu, S. (2012). Cardiovascular actions and therapeutic potential of tanshinone IIA. Atherosclerosis 220 (1), 3-10. doi: 10.1615/IntJMedMushr.v6.i1.20

Gardner, C. D., Taylor-Piliae, R. E., Kiazand, A., Nicholus, J., Rigby, A. J., and Farquhar, J. W. (2008). Effect of Ginkgo biloba (EGb 761) on treadmill walking time among adults with peripheral artery disease: a randomized clinical trial. J. Cardiopulm. Rehabil. Prev. 28 (4), 258-265. doi: 10.1097/01.HCR.0000327184.51992.b8

Gauhar, R., Hwang, S.-L., Jeong, S.-S., Kim, J.-E., Song, H., Park, D. C., et al. (2012). Heat-processed Gynostemma pentaphyllum extract improves obesity in ob/ob mice by activating AMP-activated protein kinase. Biotechnol. Lett. 34 (9), 1607-1616. doi: 10.1007/s10529-012-0944-1

Gerry, C. J., and Schreiber, S. L. (2018). Chemical probes and drug leads from advances in synthetic planning and methodology. Nat. Rev. Drug Discov. 17 (5), 333-352. doi: 10.1038/nrd.2018.53

Gou, S. H., Huang, H. F., Chen, X. Y., Liu, J., He, M., Ma, Y. Y., et al. (2016). Lipidlowering, hepatoprotective, and atheroprotective effects of the mixture Hong$\mathrm{Qu}$ and gypenosides in hyperlipidemia with NAFLD rats. J. Chin. Med. Assoc. 79 (3), 111-121. doi: 10.1016/j.jcma.2015.09.002

Gou, S. H., Liu, B. J., Han, X. F., Wang, L., Zhong, C., Liang, S., et al. (2018). Antiatherosclerotic effect of Fermentum Rubrum and Gynostemma pentaphyllum mixture in high-fat emulsion- and vitamin D3-induced atherosclerotic rats. J. Chin. Med. Assoc. 81 (5), 398-408. doi: 10.1016/j.jcma.2017.08.018

Grosse, Y., Loomis, D., Lauby-Secretan, B., El Ghissassi, F., Bouvard, V., Benbrahim-Tallaa, L., et al. (2013). Carcinogenicity of some drugs and herbal products. Lancet Oncol. 14 (9), 807-808. doi: 10.1016/S1470-2045(13) 70329-2

Guerrero-Garcia, C., and Rubio-Guerra, A. F. (2018). Combination therapy in the treatment of hypertension. Drugs Context 7, 212531. doi: 10.7573/dic.212531

Gui, Q. F., Xu, Z. R., Xu, K. Y., and Yang, Y. M. (2016). The Efficacy of GinsengRelated Therapies in Type 2 Diabetes Mellitus: An Updated Systematic Review and Meta-analysis. Medicine (Baltimore) 95 (6), e2584. doi: 10.1097/ MD.0000000000002584

Gurovic, M. S. V., Viceconte, F. R., Pereyra, M. T., Bidegain, M. A., and Cubitto, M. A. (2018). DNA damaging potential of Ganoderma lucidum extracts. J. Ethnopharmacol. 217, 83-88. doi: 10.1016/j.jep.2018.02.005 
Harvey, A. (2000). Strategies for discovering drugs from previously unexplored natural products. Drug Discovery Today 5 (7), 294-300. doi: 10.1016/S13596446(00)01511-7

Hashiguchi, M., Ohta, Y., Shimizu, M., Maruyama, J., and Mochizuki, M. (2015). Metaanalysis of the efficacy and safety of Ginkgo biloba extract for the treatment of dementia. J. Pharm. Health Care Sci. 1, 14. doi: 10.1186/s40780-015-0014-7

He, M., Pan, H., Chang, R. C., So, K. F., Brecha, N. C., and Pu, M. (2014). Activation of the $\mathrm{Nrf} / \mathrm{HO}-1$ antioxidant pathway contributes to the protective effects of Lycium barbarum polysaccharides in the rodent retina after ischemiareperfusion-induced damage. PloS One 9 (1), e84800. doi: 10.1371/ journal.pone.0084800

Heleno, S. A., Barros, L., Martins, A., Queiroz, M. J. R., Santos-Buelga, C., and Ferreira, I. (2012). Fruiting body, spores and in vitro produced mycelium of Ganoderma lucidum from Northeast Portugal: A comparative study of the antioxidant potential of phenolic and polysaccharidic extracts. Food Res. Int. 46 (1), 135-140. doi: 10.1016/j.foodres.2011.12.009

Heriansyah, T., Nurwidyaningtyas, W., Sargowo, D., Tjahjono, C. T., and Wihastuti, T. A. (2019). Polysaccharide peptide (PsP) Ganoderma lucidum: a potential inducer for vascular repair in type 2 diabetes mellitus model. Vasc. Health Risk Manage. 15, 419. doi: 10.2147/VHRM.S205996

Hikino, H., Konno, C., Mirin, Y., and Hayashi, T. (1985). Isolation and Hypoglycemic Activity of Ganoderans A and B, Glycans of Ganoderma lucidum Fruit Bodies1. Planta Med. 51 (4), 339-340. doi: 10.1055/s-2007-969507

Hong, S. Y., Kim, J. Y., Ahn, H. Y., Shin, J. H., and Kwon, O. (2012). Panax ginseng extract rich in ginsenoside protopanaxatriol attenuates blood pressure elevation in spontaneously hypertensive rats by affecting the Akt-dependent phosphorylation of endothelial nitric oxide synthase. J. Agric. Food Chem. 60 (12), 3086-3091. doi: 10.1021/jf204447y

Hong, J. M., Shin, D. H., Lim, Y. A., Lee, J. S., and Joo, I. S. (2013). Ticlopidine with Ginkgo Biloba extract: a feasible combination for patients with acute cerebral ischemia. Thromb. Res. 131 (4), e147-e153. doi: 10.1016/j.thromres.2013.01.026

Hori, T., Ridge, R. W., Tulecke, W., Del Tredici, P., Tremouillaux-Guiller, J., and Tobe, H. (1997). Ginkgo biloba: a global treasure: from biology to medicine (Japan: Springer).

Huang, T. H., Li, Y., Razmovski-Naumovski, V., Tran, V. H., Li, G. Q., Duke, C. C., et al. (2006). Gypenoside XLIX isolated from Gynostemma pentaphyllum inhibits nuclear factor-kappaB activation via a PPAR-alpha-dependent pathway. J. Biomed. Sci. 13 (4), 535-548. doi: 10.1007/s11373-006-9076-8

Huang, P., Zhang, L., Chai, C., Qian, X. C., Li, W., Li, J. S., et al. (2014). Effects of food and gender on the pharmacokinetics of ginkgolides A, B, C and bilobalide in rats after oral dosing with ginkgo terpene lactones extract. J. Pharm. Biomed. Anal. 100, 138-144. doi: 10.1016/j.jpba.2014.07.030

Huang, W. C., Chen, Y. L., Liu, H. C., Wu, S. J., and Liou, C. J. (2018). Ginkgolide $\mathrm{C}$ reduced oleic acid-induced lipid accumulation in HepG2 cells. Saudi Pharm. J. 26 (8), 1178-1184. doi: 10.1016/j.jsps.2018.07.006

Huyen, V., Phan, D., Thang, P., Hoa, N., and Östenson, C. (2010). Antidiabetic effect of Gynostemma pentaphyllum tea in randomly assigned type 2 diabetic patients. Horm. Metab. Res. 42 (05), 353-357. doi: 10.1055/s-0030-1248298

Huyen, V. T., Phan, D. V., Thang, P., Ky, P. T., Hoa, N. K., and Ostenson, C. G. (2012). Antidiabetic Effects of Add-On Gynostemma pentaphyllum Extract Therapy with Sulfonylureas in Type 2 Diabetic Patients. Evid. Based Complement. Alternat. Med. 2012, 452313. doi: 10.1155/2012/452313

Huyen, V., Phan, D., Thang, P., Hoa, N., and Östenson, C. (2013). Gynostemma pentaphyllum tea improves insulin sensitivity in type 2 diabetic patients. $J$. Nutr. Metab. 2013, 765383. doi: 10.1155/2013/765383

Hwang, S. Y., Son, D. J., Kim, I. W., Kim, D. M., Sohn, S. H., Lee, J. J., et al. (2008). Korean red ginseng attenuates hypercholesterolemia-enhanced platelet aggregation through suppression of diacylglycerol liberation in highcholesterol-diet-fed rabbits. Phytother. Res. 22 (6), 778-783. doi: 10.1002/ ptr. 2363

Isah, T. (2015). Rethinking Ginkgo biloba L.: Medicinal uses and conservation. Pharmacogn. Rev. 9 (18), 140-148. doi: 10.4103/0973-7847.162137

Izzo, A. A., and Ernst, E. (2009). Interactions between herbal medicines and prescribed drugs: an updated systematic review. Drugs 69 (13), 1777-1798. doi: 10.2165/11317010-000000000-00000

Jaggy, H., and Koch, E. (1997). Chemistry and biology of alkylphenols from Ginkgo biloba L. Pharmazie 52 (10), 735-738.
Jang, S. J., Lim, H. J., and Lim, D. Y. (2011). Inhibitory Effects of Total Ginseng Saponin on Catecholamine Secretion from the Perfused Adrenal Medulla of SHRs. J. Ginseng Res. 35 (2), 176-190. doi: 10.5142/jgr.2011.35.2.176

Jeong, Y. Y., Ryu, J. H., Shin, J. H., Kang, M. J., Kang, J. R., Han, J., et al. (2016). Comparison of Anti-Oxidant and Anti-Inflammatory Effects between Fresh and Aged Black Garlic Extracts. Molecules 21 (4), 430. doi: 10.3390/molecules21040430

Jia, Y., Li, Z. Y., Zhang, H. G., Li, H. B., Liu, Y., and Li, X. H. (2010). Panax notoginseng saponins decrease cholesterol ester via up-regulating ATPbinding cassette transporter A1 in foam cells. J. Ethnopharmacol. 132 (1), 297-302. doi: 10.1016/j.jep.2010.08.033

Johansson, B. B. (1999). Hypertension mechanisms causing stroke. Clin. Exp. Pharmacol. Physiol. 26 (7), 563-565. doi: 10.1046/j.1440-1681.1999.03081.x

Jovanovski, E., Bateman, E. A., Bhardwaj, J., Fairgrieve, C., Mucalo, I., Jenkins, A. L., et al. (2014). Effect of Rg3-enriched Korean red ginseng (Panax ginseng) on arterial stiffness and blood pressure in healthy individuals: a randomized controlled trial. $J$. Am. Soc. Hypertens. 8 (8), 537-541. doi: 10.1016/j.jash.2014.04.004

Juan, Y., and Shanzhang, Y. (1995). Observation of Effects of Gynostemma Pentaphyllum on Platelet Disaggregation in Human. J. Fujian Med. Univ. (1995) (3), 03.

Kearney, P. M., Whelton, M., Reynolds, K., Muntner, P., Whelton, P. K., and He, J. (2005). Global burden of hypertension: analysis of worldwide data. Lancet 365 (9455), 217-223. doi: 10.1016/S0140-6736(05)17741-1

Keilhoff, G., Esser, T., Titze, M., Ebmeyer, U., and Schild, L. (2017). Gynostemma pentaphyllum is neuroprotective in a rat model of cardiopulmonary resuscitation. Exp. Ther. Med. 14 (6), 6034-6046. doi: 10.3892/etm.2017.5315

Keum, Y. S., Han, S. S., Chun, K. S., Park, K. K., Park, J. H., Lee, S. K., et al. (2003), Inhibitory effects of the ginsenoside $\mathrm{Rg} 3$ on phorbol ester-induced cyclooxygenase-2 expression, NF-kappaB activation and tumor promotion. Mutat. Res., 523-524, 75-85. doi: 10.1016/s0027-5107(02)00323-8

Kiefer, D., and Pantuso, T. (2003). Panax ginseng. Am. Fam. Physician 68 (8), 1539-1542.

Kim, J. H., and Han, Y. N. (2011). Dammarane-type saponins from Gynostemma pentaphyllum. Phytochemistry 72 (11-12), 1453-1459. doi: 10.1016/ j.phytochem.2011.04.003

Kim, S. H., and Park, K. S. (2003). Effects of Panax ginseng extract on lipid metabolism in humans. Pharmacol. Res. 48 (5), 511-513. doi: 10.1016/S10436618(03)00189-0

Kim, N. D., Kang, S. Y., Kim, M. J., Park, J. H., and Schini-Kerth, V. B. (1999a). The ginsenoside $\mathrm{Rg} 3$ evokes endothelium-independent relaxation in rat aortic rings: role of K+ channels. Eur. J. Pharmacol. 367 (1), 51-57. doi: 10.1016/ s0014-2999(98)00899-1

Kim, N. D., Kang, S. Y., Park, J. H., and Schini-Kerth, V. B. (1999b). Ginsenoside $\mathrm{Rg} 3$ mediates endothelium-dependent relaxation in response to ginsenosides in rat aorta: role of $\mathrm{K}+$ channels. Eur. J. Pharmacol. 367 (1), 41-49. doi: 10.1016/s0014-2999(98)00898-x

Kim, W. Y., Kim, J. M., Han, S. B., Lee, S. K., Kim, N. D., Park, M. K., et al. (2000). Steaming of ginseng at high temperature enhances biological activity. J. Nat. Prod. 63 (12), 1702-1704. doi: 10.1021/np990152b

Kim, J. H. (2012). Cardiovascular Diseases and Panax ginseng: A Review on Molecular Mechanisms and Medical Applications. J. Ginseng Res. 36 (1), 1626. doi: 10.5142 /jgr.2012.36.1.16

Kimbel, K. H. (1992). Ginkgo biloba. Lancet 340 (8833), 1474. doi: 10.1016/01406736(92)92668-6

Kitts, D. D., Wijewickreme, A. N., and Hu, C. (2000). Antioxidant properties of a North American ginseng extract. Mol. Cell. Biochem. 203 (1-2), 1-10. doi: 10.1023/a:1007078414639

Kleijnen, J., and Knipschild, P. (1992). Ginkgo biloba. Lancet 340 (8828), 11361139. doi: $10.1016 / 0140-6736(92) 93158-\mathrm{j}$

Klein, B. E., Klein, R., and Lee, K. E. (2002). Components of the metabolic syndrome and risk of cardiovascular disease and diabetes in Beaver Dam. Diabetes Care 25 (10), 1790-1794. doi: 10.2337/diacare.25.10.1790

Klupp, N. L., Chang, D., Hawke, F., Kiat, H., Cao, H., Grant, S. J., et al. (2015). Ganoderma lucidum mushroom for the treatment of cardiovascular risk factors. Cochrane Database Syst. Rev. (2), CD007259. doi: 10.1002/ 14651858.CD007259.pub2

Klupp, N. L., Kiat, H., Bensoussan, A., Steiner, G. Z., and Chang, D. H. (2016). A double-blind, randomised, placebo-controlled trial of Ganoderma lucidum for 
the treatment of cardiovascular risk factors of metabolic syndrome. Sci. Rep. 2 (6), 29540. doi: 10.1038/srep29540

Komishon, A. M., Shishtar, E., Ha, V., Sievenpiper, J. L., de Souza, R. J., Jovanovski, E., et al. (2016). The effect of ginseng (genus Panax) on blood pressure: a systematic review and meta-analysis of randomized controlled clinical trials. J. Hum. Hypertens. 30 (10), 619-626. doi: 10.1038/jhh.2016.18

Kressmann, S., Muller, W. E., and Blume, H. H. (2002). Pharmaceutical quality of different Ginkgo biloba brands. J. Pharm. Pharmacol. 54 (5), 661-669. doi: $10.1211 / 0022357021778970$

Kudolo, G. B. (2000). The effect of 3-month ingestion of Ginkgo biloba extract on pancreatic beta-cell function in response to glucose loading in normal glucose tolerant individuals. J. Clin. Pharmacol. 40 (6), 647-654. doi: 10.1002/j.15524604.2000.tb05991.x

Kuller, L. H., Ives, D. G., Fitzpatrick, A. L., Carlson, M. C., Mercado, C., Lopez, O. L., et al. (2010). Does Ginkgo biloba reduce the risk of cardiovascular events? Circ. Cardiovasc. Qual. Outcomes 3 (1), 41-47. doi: 10.1161/circoutcomes.109.871640

Kwok, Y., Ng, K. F., Li, C. C., Lam, C. C., and Man, R. Y. (2005). A prospective, randomized, double-blind, placebo-controlled study of the platelet and global hemostatic effects of Ganoderma lucidum (Ling-Zhi) in healthy volunteers. Anesth. Analg. 101 (2), 423-426. doi: 10.1213/01.ANE.0000155286.20467.28

la Cour, B., Molgaard, P., and Yi, Z. (1995). Traditional Chinese medicine in treatment of hyperlipidaemia. J. Ethnopharmacol. 46 (2), 125-129. doi: 10.1016/0378-8741(95) 01234-5

Lacour, M., Ez-Zaher, L., and Raymond, J. (1991). Plasticity mechanisms in vestibular compensation in the cat are improved by an extract of Ginkgo biloba (EGb 761). Pharmacol. Biochem. Behav. 40 (2), 367-379. doi: 10.1016/00913057(91)90568-m

Lakatta, E. G., and Levy, D. (2003). Arterial and cardiac aging: major shareholders in cardiovascular disease enterprises: Part II: the aging heart in health: links to heart disease. Circulation 107 (2), 346-354. doi: 10.1161/01.cir.0000048893.62841.f7

Lasukova, T. V., Arbuzov, A. G., Maslov, L. N., and Burkova, V. N. (2008). [Ganoderma lucidum extract in cardiac diastolic dysfunction and irreversible cardiomyocytic damage in ischemia and reperfusion of the isolated heart]. Patol. Fiziol. Eksp. Ter. (1), 22-25.

Lasukova, T., Maslov, L., Arbuzov, A., Burkova, V., and Inisheva, L. (2015). Cardioprotective activity of Ganoderma lucidum extract during total ischemia and reperfusion of isolated heart. Bull. Exp. Biol. Med. 158 (6), 739-741. doi: $10.1007 /$ s10517-015-2851-7

Lay, S., Chiu, J.-H., Shiao, M.-S., Lui, W.-Y., and Wu, C.-W. (2003). Crude extract of Salvia miltiorrhiza and salvianolic acid B enhance in vitro angiogenesis in murine SVR endothelial cell line. Planta Med. 69 (01), 26-32. doi: 10.1055/s2003-37034

Le, L., Gao, X. L., Ding, B. X., and Yuan, B. X. (2007). [Effect of total flaveos of Gymostemma pentaphyllum on protein expression of Fas/FasL genes and TNF-alpha concentration in cultured neonatal rat cardiomyocytes with hypoxia-reoxygenation]. Zhongguo Zhong Yao Za Zhi 32 (18), 1925-1927.

Lee, C. H., and Kim, J. H. (2014). A review on the medicinal potentials of ginseng and ginsenosides on cardiovascular diseases. J. Ginseng Res. 38 (3), 161-166. doi: 10.1016/j.jgr.2014.03.001

Lee, W. M., Kim, S. D., Park, M. H., Cho, J. Y., Park, H. J., Seo, G. S., et al. (2008). Inhibitory mechanisms of dihydroginsenoside $\mathrm{Rg} 3$ in platelet aggregation: critical roles of ERK2 and cAMP. J. Pharm. Pharmacol. 60 (11), 1531-1536. doi: $10.1211 / \mathrm{jpp} / 60.11 .0015$

Lee, N. H., Yoo, S. R., Kim, H. G., Cho, J. H., and Son, C. G. (2012). Safety and tolerability of Panax ginseng root extract: a randomized, placebo-controlled, clinical trial in healthy Korean volunteers. J. Altern. Complement. Med. 18 (11), 1061-1069. doi: 10.1089/acm.2011.0591

Lee, H., Choi, J., Shin, S. S., and Yoon, M. (2016). Effects of Korean red ginseng (Panax ginseng) on obesity and adipose inflammation in ovariectomized mice. J. Ethnopharmacol. 178, 229-237. doi: 10.1016/j.jep.2015.12.017

Lee, K. H., Bae, I. Y., Park, S. I., Park, J. D., and Lee, H. G. (2016). Antihypertensive effect of Korean Red Ginseng by enrichment of ginsenoside Rg3 and argininefructose. J. Ginseng Res. 40 (3), 237-244. doi: 10.1016/j.jgr.2015.08.002

Lee, Y. H., Kim, J. H., Song, C. H., Jang, K. J., Kim, C. H., Kang, J. S., et al. (2016). Ethanol Extract of Ganoderma lucidum Augments Cellular Anti-oxidant Defense through Activation of Nrf2/HO-1. J. Pharmacopuncture 19 (1), 5969. doi: 10.3831/KPI.2016.19.008
Lee, H., Hong, Y., Tran, Q., Cho, H., Kim, M., Kim, C., et al. (2019a). A new role for the ginsenoside RG3 in antiaging via mitochondria function in ultravioletirradiated human dermal fibroblasts. J. Ginseng Res. 43 (3), 431-441. doi: $10.1016 /$ j.jgr.2018.07.003

Lee, H. S., Lim, S. M., Jung, J. I., Kim, S. M., Lee, J. K., Kim, Y. H., et al. (2019b). Gynostemma Pentaphyllum Extract Ameliorates High-Fat Diet-Induced Obesity in C57BL/6N Mice by Upregulating SIRT1. Nutrients 11 (10), 431441. doi: 10.3390/nu11102475

Li, L., and Lau, B. H. (1993). Protection of vascular endothelial cells from hydrogen peroxide-induced oxidant injury by gypenosides, saponins of Gynostemma pentaphyllum. Phytother. Res. 7 (4), 299-304. doi: 10.1002/ptr.2650070408

Li, Y., Lin, W., Huang, J., Xie, Y., and Ma, W. (2016). Anti-cancer effects of Gynostemma pentaphyllum (Thunb.) Makino (Jiaogulan). Chin. Med. 11, 43. doi: 10.1186/s13020-016-0114-9

Liang, Z., Yuan, Z., Li, G., Fu, F., and Shan, Y. (2018). Hypolipidemic, antioxidant, and antiapoptotic effects of polysaccharides extracted from Reishi Mushroom, Ganoderma lucidum (Leysser: Fr) Karst, in mice fed a high-fat diet. J. Med. Food 21 (12), 1218-1227. doi: 10.1089/jmf.2018.4182

Liao, W. I., Lin, Y. Y., Chu, S. J., Hsu, C. W., and Tsai, S. H. (2010). Bradyarrhythmia caused by ginseng in a patient with chronic kidney disease. Am. J. Emerg. Med. 28 (4), 538.e535-536. doi: 10.1016/j.ajem.2009.08.006

Lim, L. M., Tsai, N. C., Lin, M. Y., Hwang, D. Y., Lin, H. Y., Lee, J. J., et al. (2016). Hyponatremia is Associated with Fluid Imbalance and Adverse Renal Outcome in Chronic Kidney Disease Patients Treated with Diuretics. Sci. Rep. 6, 36817. doi: 10.1038/srep36817

Lin, C. C., Huang, P. C., and Lin, J. M. (2000). Antioxidant and hepatoprotective effects of Anoectochilus formosanus and Gynostemma pentaphyllum. Am. J. Chin. Med. 28 (1), 87-96. doi: 10.1142/S0192415X00000118

Lin, F. Y., Chen, Y. H., Chen, Y. L., Wu, T. C., Li, C. Y., Chen, J. W., et al. (2007). Ginkgo biloba extract inhibits endotoxin-induced human aortic smooth muscle cell proliferation via suppression of toll-like receptor 4 expression and NADPH oxidase activation. J. Agric. Food Chem. 55 (5), 1977-1984. doi: $10.1021 /$ jf062945r

Lin, M., Wang, Y. R., Zhai, X. F., Xing, S. F., and Piao, X. L. (2019). [Protective effects of flavonoids from Gynostemma pentaphyllum on oxidative damage in LLC-PK1 cells]. Zhongguo Zhong Yao Za Zhi 44 (6), 1193-1200. doi: 10.19540/ j.cnki.cjcmm.20181226.019

Liou, C. J., Lai, X. Y., Chen, Y. L., Wang, C. L., Wei, C. H., and Huang, W. C. (2015). Ginkgolide C suppresses adipogenesis in 3T3-L1 adipocytes via the AMPK signaling pathway. Evid. Based Complement. Alternat. Med. 2015, 298635. doi: 10.1155/2015/298635

Liu, A. H., Bao, Y. M., Wang, X. Y., and Zhang, Z. X. (2013). Cardio-protection by Ginkgo biloba extract 50 in rats with acute myocardial infarction is related to $\mathrm{Na}(+)-\mathrm{Ca}(2)(+)$ exchanger. Am. J. Chin. Med. 41 (4), 789-800. doi: 10.1142/ S0192415X13500535

Liu, L., Wu, J., and Kennedy, D. J. (2016). Regulation of Cardiac Remodeling by Cardiac $\mathrm{Na}(+) / \mathrm{K}(+)$-ATPase Isoforms. Front. Physiol. 7, 382. doi: 10.3389/ fphys.2016.00382

Lu, J. M., Weakley, S. M., Yang, Z., Hu, M., Yao, Q., and Chen, C. (2012). Ginsenoside Rbl directly scavenges hydroxyl radical and hypochlorous acid. Curr. Pharm. Des. 18 (38), 6339-6347. doi: 10.2174/138161212803832254

Luo, H. M., Dai, R. H., and Li, Y. (1995). [Nuclear cardiology study on effective ingredients of Astragalus membranaceus in treating heart failure]. Zhongguo Zhong Xi Yi Jie He Za Zhi 15 (12), 707-709.

Ma, X., Zhang, K., Li, H., Han, S., Ma, Z., and Tu, P. (2013). Extracts from Astragalus membranaceus limit myocardial cell death and improve cardiac function in a rat model of myocardial ischemia. J. Ethnopharmacol. 149 (3), 720-728. doi: 10.1016/j.jep.2013.07.036

Maguire, E. M., Pearce, S. W. A., and Xiao, Q. (2019). Foam cell formation: A new target for fighting atherosclerosis and cardiovascular disease. Vascul. Pharmacol. 112, 54-71. doi: 10.1016/j.vph.2018.08.002

Mahady, G. B., Gyllenhaal, C., Fong, H. H., and Farnsworth, N. R. (2000). Ginsengs: a review of safety and efficacy. Nutr. Clin. Care 3 (2), 90-101. doi: 10.1046/j.1523-5408.2000.00020.x

Mahdy, H. M., Tadros, M. G., Mohamed, M. R., Karim, A. M., and Khalifa, A. E. (2011) The effect of Ginkgo biloba extract on 3-nitropropionic acid-induced neurotoxicity in rats. Neurochem. Int. 59 (6), 770-778. doi: 10.1016/j.neuint.2011.07.012 
Mahmoudian-Sani, M. R., Hashemzadeh-Chaleshtori, M., Asadi-Samani, M., and Yang, Q. (2017). Ginkgo biloba in the treatment of tinnitus: An updated literature review. Int. Tinnitus J. 21 (1), 58-62. doi: 10.5935/0946-5448.20170011

Malek, M. A., Hoang, M. H., Jia, Y., Lee, J. H., Jun, H. J., Lee, D. H., et al. (2013). Ombuin-3-O-beta-D-glucopyranoside from Gynostemma pentaphyllum is a dual agonistic ligand of peroxisome proliferator-activated receptors alpha and delta/beta. Biochem. Biophys. Res. Commun. 430 (4), 1322-1328. doi: 10.1016/ j.bbrc.2012.12.020

Mansour, S. M., Bahgat, A. K., El-Khatib, A. S., and Khayyal, M. T. (2011). Ginkgo biloba extract (EGb 761) normalizes hypertension in $2 \mathrm{~K}, 1 \mathrm{C}$ hypertensive rats: role of antioxidant mechanisms, ACE inhibiting activity and improvement of endothelial dysfunction. Phytomedicine 18 (8-9), 641-647. doi: 10.1016/ j.phymed.2011.01.014

Mao, X.-q., Yu, F., Wang, N., Wu, Y., Zou, F., Wu, K., et al. (2009). Hypoglycemic effect of polysaccharide enriched extract of Astragalus membranaceus in diet induced insulin resistant C57BL/6J mice and its potential mechanism. Phytomedicine 16 (5), 416-425. doi: 10.1016/j.phymed.2008.12.011

Martinet, W., Coornaert, I., Puylaert, P., and De Meyer, G. R. Y. (2019). Macrophage Death as a Pharmacological Target in Atherosclerosis. Front. Pharmacol. 10, 306. doi: 10.3389/fphar.2019.00306

Mashayekh, A., Pham, D. L., Yousem, D. M., Dizon, M., Barker, P. B., and Lin, D. D. (2011). Effects of Ginkgo biloba on cerebral blood flow assessed by quantitative MR perfusion imaging: a pilot study. Neuroradiology 53 (3), 185-191. doi: 10.1007/s00234-010-0790-6

Matthews, M. K.Jr. (1998). Association of Ginkgo biloba with intracerebral hemorrhage. Neurology 50 (6), 1933-1934. doi: 10.1212/wnl.50.6.1933

Megalli, S., Aktan, F., Davies, N. M., and Roufogalis, B. D. (2005). Phytopreventative anti-hyperlipidemic effects of Gynostemma pentaphyllum in rats. J. Pharm. Pharm. Sci. 8 (3), 507-515.

Megalli, S., Davies, N. M., and Roufogalis, B. D. (2006). Anti-hyperlipidemic and hypoglycemic effects of Gynostemma pentaphyllum in the Zucker fatty rat. J. Pharm. Pharm. Sci. 9 (3), 281-291.

Mehlsen, J., Drabaek, H., Wiinberg, N., and Winther, K. (2002). Effects of a Ginkgo biloba extract on forearm haemodynamics in healthy volunteers. Clin. Physiol. Funct. Imaging 22 (6), 375-378. doi: 10.1046/j.1475-097X.2002.00445.x

Mei, N., Guo, X., Ren, Z., Kobayashi, D., Wada, K., and Guo, L. (2017). Review of Ginkgo biloba-induced toxicity, from experimental studies to human case reports. J. Environ. Sci. Health C Environ. Carcinog. Ecotoxicol. Rev. 35 (1), 128. doi: 10.1080/10590501.2016.1278298

Mensah, G. A. (2016). Hypertension and Target Organ Damage: Don't Believe Everything You Think! Ethnicity Dis. 26 (3), 275-278. doi: 10.18865/ed.26.3.275

Mesquita, T. R. R., de Jesus, I. C. G., Dos Santos, J. F., de Almeida, G. K. M., de Vasconcelos, C. M. L., Guatimosim, S., et al. (2017). Cardioprotective Action of Ginkgo biloba Extract against Sustained beta-Adrenergic Stimulation Occurs via Activation of M2/NO Pathway. Front. Pharmacol. 8, 220. doi: 10.3389/fphar.2017.00220

Miller, M. (2009). Dyslipidemia and cardiovascular risk: the importance of early prevention. QJM 102 (9), 657-667. doi: 10.1093/qjmed/hcp065

Moens, A. L., Claeys, M. J., Timmermans, J. P., and Vrints, C. J. (2005). Myocardial ischemia/reperfusion-injury, a clinical view on a complex pathophysiological process. Int. J. Cardiol. 100 (2), 179-190. doi: 10.1016/j.ijcard.2004.04.013

Mohammadi, H., Hadi, A., Kord-Varkaneh, H., Arab, A., Afshari, M., Ferguson, A. J. R., et al. (2019). Effects of ginseng supplementation on selected markers of inflammation: A systematic review and meta-analysis. Phytother. Res. 33 (8), 1991-2001. doi: 10.1002/ptr.6399

Mollah, M. L., Kim, G. S., Moon, H. K., Chung, S. K., Cheon, Y. P., Kim, J. K., et al. (2009). Antiobesity effects of wild ginseng (Panax ginseng CA Meyer) mediated by PPAR- $\gamma$, GLUT4 and LPL in ob/ob mice. Phytother. Res. 23 (2), 220-225. doi: 10.1002/ptr.2593

Moore, K. J., and Tabas, I. (2011). Macrophages in the pathogenesis of atherosclerosis. Cell 145 (3), 341-355. doi: 10.1016/j.cell.2011.04.005

Morigiwa, A., Kitabatake, K., Fujimoto, Y., and Ikekawa, N. (1986). Angiotensin converting enzyme-inhibitory triterpenes from Ganoderma lucidum. Chem. Pharm. Bull. 34 (7), 3025-3028. doi: 10.1248/cpb.34.3025

Mozaffarian, D., Benjamin, E. J., Go, A. S., Arnett, D. K., Blaha, M. J., Cushman, M., et al. (2015). Heart disease and stroke statistics-2015 update: a report from the American Heart Association. Circulation 131 (4), e29-322. doi: 10.1161/ cir.0000000000000152
Mucalo, I., Jovanovski, E., Rahelic, D., Bozikov, V., Romic, Z., and Vuksan, V. (2013). Effect of American ginseng (Panax quinquefolius L.) on arterial stiffness in subjects with type-2 diabetes and concomitant hypertension. J. Ethnopharmacol. 150 (1), 148-153. doi: 10.1016/j.jep.2013.08.015

Muller, C., Gardemann, A., Keilhoff, G., Peter, D., Wiswedel, I., and Schild, L. (2012). Prevention of free fatty acid-induced lipid accumulation, oxidative stress, and cell death in primary hepatocyte cultures by a Gynostemma pentaphyllum extract. Phytomedicine 19 (5), 395-401. doi: 10.1016/j.phymed.2011.12.002

Munoz-Durango, N., Fuentes, C. A., Castillo, A. E., Gonzalez-Gomez, L. M., Vecchiola, A., Fardella, C. E., et al. (2016). Role of the Renin-AngiotensinAldosterone System beyond Blood Pressure Regulation: Molecular and Cellular Mechanisms Involved in End-Organ Damage during Arterial Hypertension. Int. J. Mol. Sci. 17 (7), E797. doi: 10.3390/ijms17070797

Muntner, P., Carey, R. M., Gidding, S., Jones, D. W., Taler, S. J., Wright, J. T.Jr., et al. (2018). Potential U.S. Population Impact of the 2017 ACC/AHA High Blood Pressure Guideline. J. Am. Coll. Cardiol. 71 (2), 109-118. doi: 10.1016/ j.jacc.2017.10.073

Murthy, H. N., Dandin, V. S., Park, S. Y., and Paek, K. Y. (2018). Quality, safety and efficacy profiling of ginseng adventitious roots produced in vitro. Appl. Microbiol. Biotechnol. 102 (17), 7309-7317. doi: 10.1007/s00253-018-9188-x

Nadar, S. (2011). "Target organ damage in hypertension," in Hypertension (Oxford Cardiology Library). Eds. S. Nadar and G. Lip (London: Oxford University Press).

National Toxicology Program (2013). Toxicology and carcinogenesis studies of Ginkgo biloba extract (CAS No. 90045-36-6) in F344/N rats and B6C3F1/N mice (Gavage studies). Natl. Toxicol. Program Tech. Rep. Ser. (578), 1-183.

NCD Risk Factor Collaboration (NCD-RisC) (2017). Worldwide trends in blood pressure from 1975 to 2015: a pooled analysis of 1479 population-based measurement studies with 19.1 million participants. Lancet 389 (10064), 3755. doi: 10.1016/s0140-6736(16)31919-5

Newman, D. J., and Cragg, G. M. (2016). Natural Products as Sources of New Drugs from 1981 to 2014. J. Nat. Prod. 79 (3), 629-661. doi: 10.1021/ acs.jnatprod.5b01055

Ng, C., Koon, C., Cheung, D., Lam, M., Leung, P., Lau, C., et al. (2011). The antihypertensive effect of Danshen (Salvia miltiorrhiza) and Gegen (Pueraria lobata) formula in rats and its underlying mechanisms of vasorelaxation. J. Ethnopharmacol. 137 (3), 1366-1372. doi: 10.1016/j.jep.2011.08.006

Nookabkaew, S., Rangkadilok, N., and Satayavivad, J. (2006). Determination of trace elements in herbal tea products and their infusions consumed in Thailand. J. Agric. Food Chem. 54 (18), 6939-6944. doi: 10.1021/jf060571w

Noubiap, J. J., Nansseu, J. R., Nyaga, U. F., Sime, P. S., Francis, I., and Bigna, J. J. (2019). Global prevalence of resistant hypertension: a meta-analysis of data from 3.2 million patients. Heart 105 (2), 98-105. doi: 10.1136/heartjnl-2018-313599

Oh, J., Lee, H., Park, D., Ahn, J., Shin, S. S., and Yoon, M. (2012). Ginseng and its active components ginsenosides inhibit adipogenesis in 3T3-L1 cells by regulating MMP-2 and MMP-9. Evid. Based Complement. Alternat. Med. 2012, 265023. doi: $10.1155 / 2012 / 265023$

Ojeda, D., Jimenez-Ferrer, E., Zamilpa, A., Herrera-Arellano, A., Tortoriello, J., and Alvarez, L. (2010). Inhibition of angiotensin convertin enzyme (ACE) activity by the anthocyanins delphinidin- and cyanidin-3-O-sambubiosides from Hibiscus sabdariffa. J. Ethnopharmacol. 127 (1), 7-10. doi: 10.1016/ j.jep.2009.09.059

Omboni, S., and Volpe, M. (2018). Management of arterial hypertension with angiotensin receptor blockers: Current evidence and the role of olmesartan. Cardiovasc. Ther. 36 (6), e12471. doi: 10.1111/1755-5922.12471

Oparil, S., Zaman, M. A., and Calhoun, D. A. (2003). Pathogenesis of hypertension. Ann. Intern. Med. 139 (9), 761-776. doi: 10.7326/0003-4819139-9-200311040-00011

Orekhov, A. N., Sobenin, I. A., Korneev, N. V., Kirichenko, T. V., Myasoedova, V. A. Melnichenko, A. A., et al. (2013). Anti-atherosclerotic therapy based on botanicals. Recent Pat. Cardiovasc. Drug Discov. 8 (1), 56-66. doi: 10.2174/18722083113079990008

Otvos, R. A., Still, K. B. M., Somsen, G. W., Smit, A. B., and Kool, J. (2019). Drug Discovery on Natural Products: From Ion Channels to nAChRs, from Nature to Libraries, from Analytics to Assays. SLAS Discov. 24 (3), 362-385. doi: $10.1177 / 2472555218822098$

Oyama, Y., Chikahisa, L., Ueha, T., Kanemaru, K., and Noda, K. (1996). Ginkgo biloba extract protects brain neurons against oxidative stress induced by hydrogen peroxide. Brain Res. 712 (2), 349-352. doi: 10.1016/0006-8993(95)01440-3 
Ozaki, M., Kawashima, S., Yamashita, T., Hirase, T., Ohashi, Y., Inoue, N., et al. (2002). Overexpression of endothelial nitric oxide synthase attenuates cardiac hypertrophy induced by chronic isoproterenol infusion. Circ. J. 66 (9), 851856. doi: $10.1253 /$ circj.66.851

Paik, D. J., and Lee, C. H. (2015). Review of cases of patient risk associated with ginseng abuse and misuse. J. Ginseng Res. 39 (2), 89-93. doi: 10.1016/ j.jgr.2014.11.005

Pan, S. Y., Zhou, S. F., Gao, S. H., Yu, Z. L., Zhang, S. F., Tang, M. K., et al. (2013). New Perspectives on How to Discover Drugs from Herbal Medicines: CAM's Outstanding Contribution to Modern Therapeutics. Evid. Based Complement. Alternat. Med. 2013, 627375. doi: 10.1155/2013/627375

Park, M. Y., Lee, K. S., and Sung, M. K. (2005). Effects of dietary mulberry, Korean red ginseng, and banaba on glucose homeostasis in relation to PPAR-alpha, PPAR-gamma, and LPL mRNA expressions. Life Sci. 77 (26), 3344-3354. doi: 10.1016/j.lfs.2005.05.043

Park, J.-H., Lee, J.-Y., Yeo, J.-Y., Nam, J.-S., and Jung, M.-H. (2011). Antihyperlipidemic effect of ginsenoside Rg1 in type 2 diabetic mice. J. Life Sci. 21 (7), 932-938. doi: 10.5352/JLS.2011.21.7.932

Park, J. B., Kwon, S. K., Nagar, H., Jung, S. B., Jeon, B. H., Kim, C. S., et al. (2014). Rg3-enriched Korean Red Ginseng improves vascular function in spontaneously hypertensive rats. J. Ginseng Res. 38 (4), 244-250. doi: 10.1016/j.jgr.2014.05.011

Park, K. S., Park, K. I., Kim, J. W., Yun, Y. J., Kim, S. H., Lee, C. H., et al. (2014). Efficacy and safety of Korean red ginseng for cold hypersensitivity in the hands and feet: a randomized, double-blind, placebo-controlled trial. J. Ethnopharmacol. 158 (Pt A), 25-32. doi: 10.1016/j.jep.2014.09.028

Park, S. H., Huh, T. L., Kim, S. Y., Oh, M. R., Tirupathi Pichiah, P. B., Chae, S. W., et al. (2014). Antiobesity effect of Gynostemma pentaphyllum extract (actiponin): a randomized, double-blind, placebo-controlled trial. Obesity 22 (1), 63-71. doi: 10.1002/oby.20539

Perez-Vizcaino, F., Duarte, J., Jimenez, R., Santos-Buelga, C., and Osuna, A. (2009). Antihypertensive effects of the flavonoid quercetin. Pharmacol. Rep. 61 (1), 67-75. doi: 10.1016/S1734-1140(09)70008-8

Persson, I. A., Dong, L., and Persson, K. (2006). Effect of Panax ginseng extract (G115) on angiotensin-converting enzyme (ACE) activity and nitric oxide (NO) production. J. Ethnopharmacol. 105 (3), 321-325. doi: 10.1016/j.jep.2005.10.030

Qureshi, A. A., Abuirmeileh, N., Din, Z. Z., Ahmad, Y., Burger, W. C., and Elson, C. E. (1983). Suppression of cholesterogenesis and reduction of LDL cholesterol by dietary ginseng and its fractions in chicken liver. Atherosclerosis 48 (1), 81-94. doi: 10.1016/0021-9150(83)90019-9

Rakugi, H., Yu, H., Kamitani, A., Nakamura, Y., Ohishi, M., Kamide, K., et al. (1996). Links between hypertension and myocardial infarction. Am. Heart J. 132 (1 Pt 2 Su), 213-221. doi: 10.1016/S0002-8703(96)90556-6

Ras, R. T., Zock, P. L., and Draijer, R. (2011). Tea consumption enhances endothelial-dependent vasodilation; a meta-analysis. PloS One 6 (3), e16974. doi: 10.1371/journal.pone.0016974

Razavi, B. M., Amanloo, M. A., Imenshahidi, M., and Hosseinzadeh, H. (2016). The Relaxant Activity of Safranal in Isolated Rat Aortas is Mediated Predominantly via an Endothelium-Independent Mechanism: -Vasodilatory mechanism of safranal. J. Pharmacopuncture 19 (4), 329-335. doi: 10.3831/ KPI.2016.19.034

Razmovski-Naumovski, V., Huang, T. H.-W., Tran, V. H., Li, G. Q., Duke, C. C., and Roufogalis, B. D. (2005). Chemistry and pharmacology of Gynostemma Pentaphyllum. Phytochem. Rev. 4 (2-3), 197-219. doi: 10.1007/s11101-0053754-4

Reiner, Z., Laufs, U., Cosentino, F., and Landmesser, U. (2019). The year in cardiology 2018: prevention. Eur. Heart J. 40 (4), 336-344. doi: 10.1093/ eurheartj/ehy894

Ren, J., Fu, L., Nile, S. H., Zhang, J., and Kai, G. (2019). Salvia miltiorrhiza in Treating Cardiovascular Diseases: A Review on Its Pharmacological and Clinical Applications. Front. Pharmacol. 10, 753. doi: 10.3389/fphar.2019.00753

Rhee, M. Y., Kim, Y. S., Bae, J. H., Nah, D. Y., Kim, Y. K., Lee, M. M., et al. (2011). Effect of Korean red ginseng on arterial stiffness in subjects with hypertension. J. Altern. Complement. Med. 17 (1), 45-49. doi: 10.1089/acm.2010.0065

Rider, C. V., Nyska, A., Cora, M. C., Kissling, G. E., Smith, C., Travlos, G. S., et al. (2014). Toxicity and carcinogenicity studies of Ginkgo biloba extract in rat and mouse: liver, thyroid, and nose are targets. Toxicol. Pathol. 42 (5), 830-843. doi: 10.1177/0192623313501235

Ridker, P. M., Cushman, M., Stampfer, M. J., Tracy, R. P., and Hennekens, C. H. (1997). Inflammation, aspirin, and the risk of cardiovascular disease in apparently healthy men. N. Engl. J. Med. 336 (14), 973-979. doi: 10.1056/ nejm199704033361401

Ridker, P. M., Hennekens, C. H., Buring, J. E., and Rifai, N. (2000). C-reactive protein and other markers of inflammation in the prediction of cardiovascular disease in women. N. Engl. J. Med. 342 (12), 836-843. doi: 10.1056/ nejm200003233421202

Ridker, P. M., Everett, B. M., Thuren, T., MacFadyen, J. G., Chang, W. H., Ballantyne, C., et al. (2017). Antiinflammatory Therapy with Canakinumab for Atherosclerotic Disease. N. Engl. J. Med. 377 (12), 1119-1131. doi: 10.1056/ NEJMoa1707914

Rizvi, A. A. (2017). Addressing hypertension in the patient with type 2 diabetes mellitus: pathogenesis, goals, and therapeutic approach. Eur. Med. J. Diabetes 5 (1), 84-92.

Rodrigo, R., Gonzalez, J., and Paoletto, F. (2011). The role of oxidative stress in the pathophysiology of hypertension. Hypertens. Res. 34 (4), 431-440. doi: 10.1038/hr.2010.264

Russo, V., Rago, A., Russo, G. M., Calabro, R., and Nigro, G. (2011). Ginkgo biloba: an ancient tree with new arrhythmic side effects. J. Postgrad. Med. 57 (3), 221. doi: 10.4103/0022-3859.85214

Saba, E., Jeon, B. R., Jeong, D. H., Lee, K., Goo, Y. K., Kim, S. H., et al. (2016). Black ginseng extract ameliorates hypercholesterolemia in rats. J. Ginseng Res. 40 (2), 160-168. doi: 10.1016/j.jgr.2015.07.003

Saleh Al-Shehabi, T., Iratni, R., and Eid, A. H. (2016). Anti-atherosclerotic plants which modulate the phenotype of vascular smooth muscle cells. Phytomedicine 23 (11), 1068-1081. doi: 10.1016/j.phymed.2015.10.016

Samaha, A. A., Fawaz, M., Salami, A., Baydoun, S., and Eid, A. H. (2019). Antihypertensive Indigenous Lebanese Plants: Ethnopharmacology and a Clinical Trial. Biomolecules 9 (7), E292. doi: 10.3390/biom 9070292

Sanodiya, B. S., Thakur, G. S., Baghel, R. K., Prasad, G. B., and Bisen, P. S. (2009). Ganoderma lucidum: a potent pharmacological macrofungus. Curr. Pharm. Biotechnol. 10 (8), 717-742. doi: 10.2174/138920109789978757

Sargowo, D., Ovianti, N., Susilowati, E., Ubaidillah, N., Widya Nugraha, A., Vitriyaturrida, et al. (2018). The role of polysaccharide peptide of Ganoderma lucidum as a potent antioxidant against atherosclerosis in high risk and stable angina patients. Indian Heart J. 70 (5), 608-614. doi: 10.1016/j.ihj.2017.12.007

Sargowo, D., Rahmah, F. N., Nugroho, F. W., Kamila, P. A., Irawan, D., Sitio, M., et al. (2019). "The role of PSP (policakarida peptida) as anti inflammation therapy and vascular endotel cell protectors in dislipidemic patients with or without diabetes through IL1, TNF- $\alpha$, Cr Cr, EPC, CEC in STEMI and NSTEMI patients," in AIP Conference Proceedings (AIP Publishing), 020044.

Sawicka, K., Szczyrek, M., Jastrzębska, I., Prasał, M., Zwolak, A., and Daniluk, J. (2011). Hypertension - The Silent Killer. J. Pre Clin. Clin. Res. 5 (2), 43-46.

Schild, L., Chen, B. H., Makarov, P., Kattengell, K., Heinitz, K., and Keilhoff, G. (2010). Selective induction of apoptosis in glioma tumour cells by a Gynostemma pentaphyllum extract. Phytomedicine 17 (8-9), 589-597. doi: 10.1016/j.phymed.2009.12.002

Seldenrijk, A., van Hout, H. P., van Marwijk, H. W., de Groot, E., Gort, J., Rustemeijer, C., et al. (2011). Depression, anxiety, and arterial stiffness. Biol. Psychiatry 69 (8), 795-803. doi: 10.1016/j.biopsych.2010.12.034

Seyedemadi, P., Rahnema, M., Bigdeli, M. R., Oryan, S., and Rafati, H. (2016). The Neuroprotective Effect of Rosemary (Rosmarinus officinalis L.) Hydroalcoholic Extract on Cerebral Ischemic Tolerance in Experimental Stroke. Iran J. Pharm. Res. 15 (4), 875-883.

Shah, B. R., Retnakaran, R., and Booth, G. L. (2008). Increased risk of cardiovascular disease in young women following gestational diabetes mellitus. Diabetes Care 31 (8), 1668-1669. doi: 10.2337/dc08-0706

Shergis, J. L., Zhang, A. L., Zhou, W., and Xue, C. C. (2013). Panax ginseng in randomised controlled trials: a systematic review. Phytother. Res. 27 (7), 949965. doi: $10.1002 /$ ptr.4832

Shevelev, O. B., Seryapina, A. A., Zavjalov, E. L., Gerlinskaya, L. A., Goryachkovskaya, T. N., Slynko, N. M., et al. (2018). Hypotensive and neurometabolic effects of intragastric Reishi (Ganoderma lucidum) 
administration in hypertensive ISIAH rat strain. Phytomedicine 41, 1-6. doi: 10.1016/j.phymed.2018.01.013

Shi, Y. L., James, A. E., Benzie, I. F., and Buswell, J. A. (2002). Mushroom-derived preparations in the prevention of $\mathrm{H} 2 \mathrm{O} 2$-induced oxidative damage to cellular DNA. Teratog. Carcinog. Mutagen. 22 (2), 103-111. doi: 10.1002/tcm.10008

Shi, W.-L., Han, H., Chen, G.-Z., Chen, X., Hong, Y.-K., Chen, L.-K., et al. (2010). Extraction, characterization of the polysaccharide extracts from Se-enriched G. lucidum (Se-GLP) and its inhibition against oxidative damage in ischemic reperfusion mice. Carbohydr. Polym. 80 (3), 774-778. doi: 10.1016/ j.carbpol.2009.12.027

Shih-Chen, L., Smith, F., and Stuart, G. (1973). Chinese medicinal herbs. (San Francisco: Georgetown Press).

Shin, S. S., and Yoon, M. (2018). Korean red ginseng (Panax ginseng) inhibits obesity and improves lipid metabolism in high fat diet-fed castrated mice. J. Ethnopharmacol. 210, 80-87. doi: 10.1016/j.jep.2017.08.032

Shin, W., Yoon, J., Oh, G. T., and Ryoo, S. (2013). Korean red ginseng inhibits arginase and contributes to endotheliumdependent vasorelaxation through endothelial nitric oxide synthase coupling. J. Ginseng Res. 37 (1), 64-73. doi: 10.5142/jgr.2013.37.64

Shin, Y. M., Jung, H. J., Choi, W. Y., and Lim, C. J. (2013). Antioxidative, antiinflammatory, and matrix metalloproteinase inhibitory activities of 20 (S)ginsenoside Rg3 in cultured mammalian cell lines. Mol. Biol. Rep. 40 (1), 269-279. doi: 10.1007/s11033-012-2058-1

Shishtar, E., Jovanovski, E., Jenkins, A., and Vuksan, V. (2014a). Effects of Korean White Ginseng (Panax Ginseng CA Meyer) on vascular and glycemic health in type 2 diabetes: results of a randomized, double blind, placebo-controlled, multiple-crossover, acute dose escalation trial. Clin. Nutr. Res. 3 (2), 89-97. doi: 10.7762/cnr.2014.3.2.89

Shishtar, E., Sievenpiper, J. L., Djedovic, V., Cozma, A. I., Ha, V., Jayalath, V. H., et al. (2014b). The effect of ginseng (the genus panax) on glycemic control: a systematic review and meta-analysis of randomized controlled clinical trials. PloS One 9 (9), e107391. doi: 10.1371/journal.pone.0107391

Siegel, R. K. (1979). Ginseng abuse syndrome. Problems with the panacea. JAMA 241 (15), 1614-1615. doi: 10.1001/jama.1979.03290410046024

Singh, R. B., and Niaz, M. A. (1996). Antioxidants, oxidants and free radical stress in cardiovascular disease. J. Assoc. Physicians India 44 (1), 43-48.

Singh, B., Kaur, P., Gopichand,, Singh, R. D., and Ahuja, P. S. (2008). Biology and chemistry of Ginkgo biloba. Fitoterapia 79 (6), 401-418. doi: 10.1016/ j.fitote.2008.05.007

Singh, R. K., Lui, E., Wright, D., Taylor, A., and Bakovic, M. (2017). Alcohol extract of North American ginseng (Panax quinquefolius) reduces fatty liver, dyslipidemia, and other complications of metabolic syndrome in a mouse model. Can. J. Physiol. Pharmacol. 95 (9), 1046-1057. doi: 10.1139/cjpp-2016-0510

Smina, T. P., Mathew, J., Janardhanan, K. K., and Devasagayam, T. P. (2011). Antioxidant activity and toxicity profile of total triterpenes isolated from Ganoderma lucidum (Fr.) P. Karst occurring in South India. Environ. Toxicol. Pharmacol. 32 (3), 438-446. doi: 10.1016/j.etap.2011.08.011

Song, S. W., Kim, H. N., Shim, J. Y., Yoo, B. Y., Kim, D. H., Lee, S. H., et al. (2018). Safety and tolerability of Korean Red Ginseng in healthy adults: a multicenter, double-blind, randomized, placebo-controlled trial. J. Ginseng Res. 42 (4), 571576. doi: 10.1016/j.jgr.2018.07.002

Sowers, J. R. (2003). Obesity as a cardiovascular risk factor. Am. J. Med. 115 (8), 37-41. doi: 10.1016/j.amjmed.2003.08.012

Srichana, D., Taengtip, R., and Kondo, S. (2011). Antimicrobial activity of Gynostemma pentaphyllum extracts against fungi producing aflatoxin and fumonisin and bacteria causing diarrheal disease. Southeast Asian J. Trop. Med. Public Health 42 (3), 704-710.

Stewart, M. H., Lavie, C. J., and Ventura, H. O. (2019). Emerging Therapy in Hypertension. Curr. Hypertens. Rep. 21 (3), 23. doi: 10.1007/s11906-019-0923-1

Sun, T., Wang, X., and Xu, H. (2015). Ginkgo Biloba extract for angina pectoris: a systematic review. Chin. J. Integr. Med. 21 (7), 542-550. doi: 10.1007/s11655015-2070-0

Sun, M., Chai, L., Lu, F., Zhao, Y., Li, Q., Cui, B., et al. (2018). Efficacy and Safety of Ginkgo Biloba Pills for Coronary Heart Disease with Impaired Glucose Regulation: Study Protocol for a Series of N-of-1 Randomized, DoubleBlind, Placebo-Controlled Trials. Evid. Based Complement. Alternat. Med. 2018, 7571629. doi: 10.1155/2018/7571629
Sun, Y. E., Wang, W., and Qin, J. (2018). Anti-hyperlipidemia of garlic by reducing the level of total cholesterol and low-density lipoprotein: A meta-analysis. Medicine (Baltimore) 97 (18), e0255. doi: 10.1097/MD.0000000000010255

Susalit, E., Agus, N., Effendi, I., Tjandrawinata, R. R., Nofiarny, D., PerrinjaquetMoccetti, T., et al. (2011). Olive (Olea europaea) leaf extract effective in patients with stage-1 hypertension: comparison with Captopril. Phytomedicine 18 (4), 251-258. doi: 10.1016/j.phymed.2010.08.016

Tabas, I., Williams, K. J., and Boren, J. (2007). Subendothelial lipoprotein retention as the initiating process in atherosclerosis: update and therapeutic implications. Circulation 116 (16), 1832-1844. doi: 10.1161/circulationaha.106.676890

Tabassum, N., and Ahmad, F. (2011). Role of natural herbs in the treatment of hypertension. Pharmacogn. Rev. 5 (9), 30-40. doi: 10.4103/0973-7847.79097

Tachjian, A., Maria, V., and Jahangir, A. (2010). Use of herbal products and potential interactions in patients with cardiovascular diseases. J. Am. Coll. Cardiol. 55 (6), 515-525. doi: 10.1016/j.jacc.2009.07.074

Tada, Y., Kagota, S., Kubota, Y., Nejime, N., Nakamura, K., Kunitomo, M., et al. (2008). Long-term feeding of Ginkgo biloba extract impairs peripheral circulation and hepatic function in aged spontaneously hypertensive rats. Biol. Pharm. Bull. 31 (1), 68-72. doi: 10.1248/bpb.31.68

Tan, M. S., Yu, J. T., Tan, C. C., Wang, H. F., Meng, X. F., Wang, C., et al. (2015). Efficacy and adverse effects of ginkgo biloba for cognitive impairment and dementia: a systematic review and meta-analysis. J. Alzheimers Dis. 43 (2), 589-603. doi: 10.3233/JAD-140837

Tanner, M. A., Bu, X., Steimle, J. A., and Myers, P. R. (1999). The direct release of nitric oxide by gypenosides derived from the herb Gynostemma pentaphyllum. Nitric. Oxide 3 (5), 359-365. doi: 10.1006/niox.1999.0245

Tao, J., and Feng, K. Y. (1990). Experimental and clinical studies on inhibitory effect of ganoderma lucidum on platelet aggregation. J. Tongji Med. Univ. 10 (4), 240-243. doi: 10.1007/bf02887938

Tedgui, A., and Mallat, Z. (2006). Cytokines in atherosclerosis: pathogenic and regulatory pathways. Physiol. Rev. 86 (2), 515-581. doi: 10.1152/physrev.00024.2005

Thomson, M., Al-Qattan, K. K., Js, D., and Ali, M. (2016). Anti-diabetic and antioxidant potential of aged garlic extract (AGE) in streptozotocin-induced diabetic rats. BMC Complement. Altern. Med. 16, 17. doi: 10.1186/s12906-016-0992-5

Toda, N., Ayajiki, K., Fujioka, H., and Okamura, T. (2001). Ginsenoside potentiates NO-mediated neurogenic vasodilatation of monkey cerebral arteries. J. Ethnopharmacol. 76 (1), 109-113. doi: 10.1016/s0378-8741(01)00217-3

Tomoda, M., Gonda, R., Kasahara, Y., and Hikino, H. (1986). Glycan structures of ganoderans $\mathrm{b}$ and $\mathrm{c}$, hypoglycemic glycans of ganoderma lucidum fruit bodies. Phytochemistry 25 (12), 2817-2820. doi: 10.1016/S0031-9422(00)83748-6

Torbey, E., Abi Rafeh, N., Khoueiry, G., Kowalski, M., and Bekheit, S. (2011). Ginseng: a potential cause of long QT. J. Electrocardiol. 44 (3), 357-358. doi: 10.1016/j.jelectrocard.2010.08.007

Toth, P. P. (2007). Making a case for quantitative assessment of cardiovascular risk. J. Clin. Lipidol. 1 (4), 234-241. doi: 10.1016/j.jacl.2007.07.002

Triantafyllidi, H., Tzortzis, S., Lekakis, J., Ikonomidis, I., Arvaniti, C., Trivilou, P., et al. (2010). Association of target organ damage with three arterial stiffness indexes according to blood pressure dipping status in untreated hypertensive patients. Am. J. Hypertens. 23 (12), 1265-1272. doi: 10.1038/ajh.2010.156

Tsutsui, M., Nakata, S., Shimokawa, H., Otsuji, Y., and Yanagihara, N. (2008). Spontaneous myocardial infarction and nitric oxide synthase. Trends Cardiovasc. Med. 18 (8), 275-279. doi: 10.1016/j.tcm.2008.12.002

Ubaidillah, N., Widya, A., Vittryaturida,, Siwi, K., Failasufi, M., Ramadhan, F., et al. (2016). "The novel effect of polysaccharide peptides ganoderma lucidum in endothelial dysfuction on stable angina patients," in European Heart Journal Supplements (Oxford, England: Oxford Univ Press), B39-B39.

Ude, C., Schubert-Zsilavecz, M., and Wurglics, M. (2013). Ginkgo biloba extracts: a review of the pharmacokinetics of the active ingredients. Clin. Pharmacokinet. 52 (9), 727-749. doi: 10.1007/s40262-013-0074-5

Valli, G., and Giardina, E. G. (2002). Benefits, adverse effects and drug interactions of herbal therapies with cardiovascular effects. J. Am. Coll. Cardiol. 39 (7), 1083-1095. doi: 10.1016/s0735-1097(02)01749-7

Virmani, R., Burke, A. P., Kolodgie, F. D., and Farb, A. (2002). Vulnerable plaque: the pathology of unstable coronary lesions. J. Interv. Cardiol. 15 (6), 439-446. doi: 10.1111/j.1540-8183.2002.tb01087.x

Vogelzangs, N., Seldenrijk, A., Beekman, A. T., van Hout, H. P., de Jonge, P., and Penninx, B. W. (2010). Cardiovascular disease in persons with depressive and 
anxiety disorders. J. Affect. Disord. 125 (1-3), 241-248. doi: 10.1016/ j.jad.2010.02.112

Vogler, B. K., Pittler, M. H., and Ernst, E. (1999). The efficacy of ginseng. A systematic review of randomised clinical trials. Eur. J. Clin. Pharmacol. 55 (8), 567-575. doi: 10.1007/s002280050674

Wachtel-Galor, S., Tomlinson, B., and Benzie, I. F. (2004). Ganoderma lucidum ("Lingzhi"), a Chinese medicinal mushroom: biomarker responses in a controlled human supplementation study. Br. J. Nutr. 91 (2), 263-269. doi: 10.1079/BJN20041039

Wachtel-Galor, S., Wong, W.-c., Choi, S.-W., and Benzie, I. F. (2010). Antioxidant Power and DNA Repair Effects of Lingzhi or Reishi Medicinal Mushroom, Ganoderma lucidum (W. Curt.: Fr.) P. Karst.(Aphyllophoromycetideae), in Human Acute Post-ingestion Study. Int. J. Med. Mushrooms 12 (4), 359-366. doi: 10.1615/IntJMedMushr.v12.i4.30

Wada, K., Ishigaki, S., Ueda, K., Take, Y., Sasaki, K., Sakata, M., et al. (1988). Studies on the constitution of edible and medicinal plants. I. Isolation and identification of 4O-methylpyridoxine, toxic principle from the seed of Ginkgo biloba L. Chem. Pharm. Bull. 36 (5), 1779-1782. doi: 10.1248/cpb.36.1779

Wada, K. (2005). [Ginkgo seed food poisoning]. Chudoku Kenkyu 18 (1), 11-16.

Wang, J., and Xiong, X. (2012). Outcome measures of chinese herbal medicine for hypertension: an overview of systematic reviews. Evid. Based Complement. Alternat. Med. 2012, 697237. doi: 10.1155/2012/697237

Wang, M., Wang, F., Wang, Y., Ma, X., Zhao, M., and Zhao, C. (2013). Metabonomics study of the therapeutic mechanism of Gynostemma pentaphyllum and atorvastatin for hyperlipidemia in rats. PloS One 8 (11), e78731. doi: 10.1371/journal.pone.0078731

Wang, F., Zhou, Z., Ren, X., Wang, Y., Yang, R., Luo, J., et al. (2015). Effect of Ganoderma lucidum spores intervention on glucose and lipid metabolism gene expression profiles in type 2 diabetic rats. Lipids Health Dis. 14, 49. doi: 10.1186/s12944-015-0045-y

Wang, Z., Zhang, J., Ren, T., and Dong, Z. (2016). Targeted metabolomic profiling of cardioprotective effect of Ginkgo biloba L. extract on myocardial ischemia in rats. Phytomedicine 23 (6), 621-631. doi: 10.1016/j.phymed.2016.03.005

Wang, Y. R., Yang, K., Cui, W. Y., and Piao, X. L. (2018). [Effects of flavonoids from Gynostemma pentaphyllum on A549 cells damaged by hydrogen peroxide]. Zhongguo Zhong Yao Za Zhi 43 (5), 1014-1020. doi: 10.19540/ j.cnki.cjcmm.2018.0034

Wang, Z., Zhao, X., Liu, X., Lu, W., Jia, S., Hong, T., et al. (2019). Anti-diabetic activity evaluation of a polysaccharide extracted from Gynostemma pentaphyllum. Int. J. Biol. Macromol. 126, 209-214. doi: 10.1016/j.ijbiomac.2018.12.231

Weber, C., and Noels, H. (2011). Atherosclerosis: current pathogenesis and therapeutic options. Nat. Med. 17 (11), 1410-1422. doi: 10.1038/nm.2538

Weber, M. A., Schiffrin, E. L., White, W. B., Mann, S., Lindholm, L. H., Kenerson, J. G., et al. (2014). Clinical practice guidelines for the management of hypertension in the community a statement by the American Society of Hypertension and the International Society of Hypertension. J. Hypertens. 32 (1), 3-15. doi: 10.1097/ hjh.0000000000000065

Weinmann, S., Roll, S., Schwarzbach, C., Vauth, C., and Willich, S. N. (2010). Effects of Ginkgo biloba in dementia: systematic review and meta-analysis. BMC Geriatr. 10, 14. doi: 10.1186/1471-2318-10-14

Wicks, S. M., Tong, R., Wang, C. Z., O'Connor, M., Karrison, T., Li, S., et al. (2007). Safety and tolerability of Ganoderma lucidum in healthy subjects: a double-blind randomized placebo-controlled trial. Am. J. Chin. Med. 35 (3), 407-414. doi: 10.1142/S0192415X07004928

Widya, A., Ubaidillah, N., Vittryaturida,, Siwi, K., Failasufi, M., Ramadhan, F., et al. (2016). "An experimental study of beta-D-Glucans exctract of Ganoderma lucidum polysaccharide peptides as anti-inflammation and antioxidant in patients at high-risk of atherosclerosis," in European Heart Journal Supplements (Oxford, England: Oxford Univ Press), B19-B19.

Wihastuti, T. A., and Heriansyah, T. (2017). The inhibitory effects of polysaccharide peptides ( $\mathrm{Ps} \mathrm{P}$ ) of Ganoderma lucidum against atherosclerosis in rats with dyslipidemia. Heart Int. 12 (1), e1-e7. doi: 10.5301/heartint.5000234

Witztum, J. L., and Berliner, J. A. (1998). Oxidized phospholipids and isoprostanes in atherosclerosis. Curr. Opin. Lipidol. 9 (5), 441-448. doi: 10.1097/00041433199810000-00008

Wong, W. Y., Lee, M. M., Chan, B. D., Ma, V. W., Zhang, W., Yip, T. T., et al. (2017). Gynostemma pentaphyllum saponins attenuate inflammation in vitro and in vivo by inhibition of NF-kappaB and STAT3 signaling. Oncotarget 8 (50), 87401-87414. doi: 10.18632/oncotarget.20997

World Health Organization (2017). Cardiovascular Diseases (CVDs) (World Health Organization). Available: https://www.who.int/news-room/factsheets/detail/cardiovascular-diseases-(cvds) [Accessed 26-06-2019].

Wu, Q., Li, Y., Peng, K., Wang, X. L., Ding, Z., Liu, L., et al. (2019). Isolation and Characterization of Three Antihypertension Peptides from the Mycelia of Ganoderma Lucidum (Agaricomycetes). J. Agric. Food Chem. 67 (29), 81498159. doi: $10.1021 /$ acs.jafc.9b02276

Xie, Y. Z., Yang, F., Tan, W., Li, X., Jiao, C., Huang, R., et al. (2016). The anticancer components of Ganoderma lucidum possesses cardiovascular protective effect by regulating circular RNA expression. Oncoscience 3 (7-8), 203-207. doi: 10.18632/oncoscience.316

Xiong, X. J., Liu, W., Yang, X. C., Feng, B., Zhang, Y. Q., Li, S. J., et al. (2014). Ginkgo biloba extract for essential hypertension: a systemic review. Phytomedicine 21 (10), 1131-1136. doi: 10.1016/j.phymed.2014.04.024

Yamamoto, M., Uemura, T., Nakama, S., Uemiya, M., and Kumagai, A. (1983). Serum HDL-cholesterol-increasing and fatty liver-improving actions of Panax ginseng in high cholesterol diet-fed rats with clinical effect on hyperlipidemia in man. Am. J. Chin. Med. 11 (1-4), 96-101. doi: 10.1142/S0192415X83000161

Yan, W., Niu, Y., Lv, J., Xie, Z., Jin, L., Yao, W., et al. (2013). Characterization of a heteropolysaccharide isolated from diploid Gynostemma pentaphyllum Makino. Carbohydr. Polym. 92 (2), 2111-2117. doi: 10.1016/j.carbpol.2012.11.074

Yang, J.-W., and Kim, S. S. (2015). Ginsenoside Rc promotes anti-adipogenic activity on 3T3-L1 adipocytes by down-regulating C/EBP $\alpha$ and PPAR $\gamma$. Molecules 20 (1), 1293-1303. doi: 10.3390/molecules20011293

Yang, T. Y., Wei, J. C., Lee, M. Y., Chen, C. M., and Ueng, K. C. (2012). A randomized, double-blind, placebo-controlled study to evaluate the efficacy and tolerability of Fufang Danshen (Salvia miltiorrhiza) as add-on antihypertensive therapy in Taiwanese patients with uncontrolled hypertension. Phytother. Res. 26 (2), 291-298. doi: 10.1002/ptr.3548

Yang, K., Zhang, H., Luo, Y., Zhang, J., Wang, M., Liao, P., et al. (2017). Gypenoside XVII Prevents atherosclerosis by attenuating endothelial apoptosis and oxidative stress: Insight into the ER $\alpha$-mediated PI3K/Akt pathway. Int. J. Mol. Sci. 18 (2), 77. doi: 10.3390/ijms18020077

Yeh, G. Y., Davis, R. B., and Phillips, R. S. (2006). Use of complementary therapies in patients with cardiovascular disease. Am. J. Cardiol. 98 (5), 673-680. doi: 10.1016/j.amjcard.2006.03.051

Yeo, J., Kang, Y. J., Jeon, S. M., Jung, U. J., Lee, M. K., Song, H., et al. (2008). Potential hypoglycemic effect of an ethanol extract of Gynostemma pentaphyllum in C57BL/KsJ-db/db mice. J. Med. Food 11 (4), 709-716. doi: 10.1089/jmf.2007.0148

Yu, H., Guan, Q., Guo, L., Zhang, H., Pang, X., Cheng, Y., et al. (2016a). Gypenosides alleviate myocardial ischemia-reperfusion injury via attenuation of oxidative stress and preservation of mitochondrial function in rat heart. Cell Stress Chaperones 21 (3), 429-437. doi: 10.1007/s12192-016-0669-5

Yu, H., Zhang, H., Zhao, W., Guo, L., Li, X., Li, Y., et al. (2016b). Gypenoside Protects against Myocardial Ischemia-Reperfusion Injury by Inhibiting Cardiomyocytes Apoptosis via Inhibition of CHOP Pathway and Activation of PI3K/Akt Pathway In Vivo and In Vitro. Cell Physiol. Biochem. 39 (1), 123 136. doi: $10.1159 / 000445611$

Yuan, C. S., Wei, G., Dey, L., Karrison, T., Nahlik, L., Maleckar, S., et al. (2004). Brief communication: American ginseng reduces warfarin's effect in healthy patients: a randomized, controlled Trial. Ann. Intern. Med. 141 (1), 23-27. doi: 10.7326/0003-4819-141-1-200407060-00011

Yuan, H., Ma, Q., Ye, L., and Piao, G. (2016). The Traditional Medicine and Modern Medicine from Natural Products. Molecules 21 (5), E559. doi: 10.3390/ molecules 21050559

Zeng, Z., Zhu, J., Chen, L., Wen, W., and Yu, R. (2013). Biosynthesis pathways of ginkgolides. Pharmacogn. Rev. 7 (13), 47-52. doi: 10.4103/0973-7847.112848

Zhang, W.-D., Chen, H., Zhang, C., Liu, R.-H., Li, H.-L., and Chen, H.-Z. (2006). Astragaloside IV from Astragalus membranaceus shows cardioprotection during myocardial ischemia in vivo and in vitro. Planta Med. 72 (01), 4-8. doi: 10.1055/s-2005-873126

Zhang, L., Yang, Y., Wang, Y., and Gao, X. (2011). Astragalus membranaceus extract promotes neovascularisation by VEGF pathway in rat model of ischemic injury. Pharmazie 66 (2), 144-150. 
Zhang, J., Gao, X., Pan, Y., Xu, N., and Jia, L. (2016). Toxicology and immunology of Ganoderma lucidum polysaccharides in Kunming mice and Wistar rats. Int. J. Biol. Macromol. 85, 302-310. doi: 10.1016/j.ijbiomac.2015.12.090

Zhang, X., Xiao, C., and Liu, H. (2018). Ganoderic Acid A Protects Rat H9c2 Cardiomyocytes from Hypoxia-Induced Injury via Up-Regulating miR-1825p. Cell Physiol. Biochem. 50 (6), 2086-2096. doi: 10.1159/000495053

Zhang, X., Su, H., Yang, J., Feng, L., Li, Z., and Zhao, G. (2019). Population genetic structure, migration, and polyploidy origin of a medicinal species Gynostemma pentaphyllum (Cucurbitaceae). Ecol. Evol. 9 (19), 11145-11170. doi: 10.1002/ece3.5618

Zhao, G.-R., Xiang, Z.-J., Ye, T.-X., Yuan, Y.-J., and Guo, Z.-X. (2006). Antioxidant activities of Salvia miltiorrhiza and Panax notoginseng. Food Chem. 99 (4), 767-774. doi: 10.1016/j.foodchem.2005.09.002

Zheng, X. J. (2004). [Composition analysis and dominance test of three kinds of raw variety of Gynostemma pentaphyllum]. Zhongguo Zhong Yao Za Zhi 29 (4), 317-319.
Zhou, J., Fan, Y., Kong, J., Wu, D., and Hu, Z. (2000). Effects of components isolated from Astragalus membranaceus Bunge on cardiac function injured by myocardial ischemia reperfusion in rats. Zhongguo Zhong Yao Za Zhi 25 (5), 300-302.

Conflict of Interest: The authors declare that the research was conducted in the absence of any commercial or financial relationships that could be construed as a potential conflict of interest.

Copyright (c) 2020 Shaito, Thuan, Phu, Nguyen, Hasan, Halabi, Abdelhady, Nasrallah, Eid and Pintus. This is an open-access article distributed under the terms of the Creative Commons Attribution License (CC BY). The use, distribution or reproduction in other forums is permitted, provided the original author(s) and the copyright owner(s) are credited and that the original publication in this journal is cited, in accordance with accepted academic practice. No use, distribution or reproduction is permitted which does not comply with these terms. 